\title{
Mass-conserving diffusion-based dynamics on graphs
}

\author{
J. M BUDD and Y. VAN GENNIP \\ Delft Institute of Applied Mathematics (DIAM), Technische Universiteit Delft, Delft, The Netherlands \\ e-mails:j.m.budd-1@tudelft.nl; y.vangennip@tudelft.nl
}

(Received 20 June 2020; revised 5 January 2021; accepted 11 March 2021; first published online 14 April 2021)

\begin{abstract}
An emerging technique in image segmentation, semi-supervised learning and general classification problems concerns the use of phase-separating flows defined on finite graphs. This technique was pioneered in Bertozzi and Flenner (2012, Multiscale Modeling and Simulation 10(3), 1090-1118), which used the Allen-Cahn flow on a graph, and was then extended in Merkurjev et al. (2013, SIAM J. Imaging Sci. 6(4), 1903-1930) using instead the Merriman-Bence-Osher (MBO) scheme on a graph. In previous work by the authors, Budd and Van Gennip (2020, SIAM J. Math. Anal. 52(5), 4101-4139), we gave a theoretical justification for this use of the MBO scheme in place of Allen-Cahn flow, showing that the MBO scheme is a special case of a 'semi-discrete' numerical scheme for Allen-Cahn flow. In this paper, we extend this earlier work, showing that this link via the semi-discrete scheme is robust to passing to the mass-conserving case. Inspired by Rubinstein and Sternberg (1992, IMA J. Appl. Math. 48, 249-264), we define a mass-conserving Allen-Cahn equation on a graph. Then, with the help of the tools of convex optimisation, we show that our earlier machinery can be applied to derive the mass-conserving MBO scheme on a graph as a special case of a semi-discrete scheme for mass-conserving Allen-Cahn. We give a theoretical analysis of this flow and scheme, proving various desired properties like existence and uniqueness of the flow and convergence of the scheme, and also show that the semi-discrete scheme yields a choice function for solutions to the mass-conserving MBO scheme.
\end{abstract}

Key words: Allen-Cahn equation, threshold dynamics, graph dynamics, mass constrained motion, convex optimisation

2020 Mathematics Subject Classification: Primary: 34B45, 35R02; Secondary: 34A12, 65N12, $05 \mathrm{C} 99$

\section{Introduction}

In this paper, we will investigate variants of the Allen-Cahn equation and Merriman-BenceOsher (MBO) scheme on a graph, modified to ensure that mass is conserved along trajectories. First, we formulate on a graph the mass-conserving Allen-Cahn flow devised by Rubinstein and Sternberg [34], noticing that mass conservation continues to hold in the discrete setting. Next, following our earlier work in [14] and drawing on work in Van Gennip [37], we show that formulation of a mass-conserving $\mathrm{MBO}$ scheme arises naturally as a special case of a semidiscrete scheme for the mass-conserving Allen-Cahn flow with the double-obstacle potential. We then examine various theoretical properties of this mass-conserving semi-discrete scheme. 


\subsection{Contributions of this work}

In this paper, we have:

(i) Following [34], defined a mass-conserving graph Allen-Cahn flow with double-obstacle potential (Definition 3.5) and proved that it conserves mass (Proposition 3.3).

(ii) Extended the analysis in [14, Section 3] to this new flow, proving a weak form, an explicit form, and uniqueness and existence theory for this flow (Theorems 3.6, 3.7, 3.8 and 3.9, respectively) and, via the semi-discrete scheme, proved that solutions exhibit monotonic decrease of the Ginzburg-Landau energy and Lipschitz regularity (Theorems 5.9 and 5.11, respectively).

(iii) Defined a mass-conserving semi-discrete scheme for this flow (Definition 4.1) and, as in [14, Theorem 4.2], proved that this scheme is equivalent to a variational scheme of which the MBO scheme is a special case (Theorems 4.3 and 4.22).

(iv) Used the tools of convex optimisation to characterise the solutions of this variational scheme (Theorems 4.16 and 4.19) and proved that in the MBO limit, the mass-conserving semi-discrete solutions converge to an $\mathrm{MBO}$ solution, providing a choice function for the mass-conserving MBO solutions (Theorem 4.21).

(v) Following [14, Sections 4.2 and 5], derived a Lyapunov functional for the mass-conserving semi-discrete scheme (Theorem 4.26) and proved convergence of the scheme to the AllenCahn trajectory (Theorem 5.7), giving a novel proof of a key lemma.

Though we worked in the framework of [14], this paper extends upon that paper in a number of key ways. Most directly, we have shown a new result, that shows that the semi-discrete scheme link between the Allen-Cahn flow and the MBO scheme in the non-mass-conserving case is robust in the presence of a mass constraint. Moreover, this was not a trivial extension: the mass conservation condition substantially increased the difficulty of demonstrating some of the key results. In particular, finding the solutions of the variational form and thereby proving the equivalence to the semi-discrete scheme for Allen-Cahn, which are both fairly straightforward in the ordinary case, required a substantial employment of the tools of convex optimisation. Other results, such as Theorems 3.6 and 4.21, also required non-trivial extensions to the proofs of their counterparts in [14] (indeed, the latter being in that context sufficiently clear as to not be needed to be stated). Furthermore, for the proof of convergence, we have exhibited a novel proof technique for one of the key lemmas.

We now give a brief overview of the rest of this paper.

In Section 1.2, we describe how this work fits in against the background of previous literature. In Section 2, we outline our notation and key definitions and then briefly describe the link from [14] that we shall be extending to the mass-conserving case in this paper. In Section 3, we exhibit contributions (i) and (ii). In Section 4, we exhibit contributions (iii), (iv) and the first half of contribution (v). Finally, in Section 5, we exhibit the second half of contribution (v) and then use this convergence to prove monotonicity of the Ginzburg-Landau functional along massconserving Allen-Cahn trajectories and prove the Lipschitz regularity of those trajectories.

\subsection{Background}

The primary background for this work is [14], in which the authors developed a general framework for linking graph Allen-Cahn flow and the graph MBO scheme via a semi-discrete 
scheme. We showed that the MBO scheme was a special time discretisation of Allen-Cahn flow with a double-obstacle potential and investigated properties of this Allen-Cahn flow and time discretisation scheme. This paper will follow that framework, introducing a mass constraint.

Mass conservation (a.k.a. volume preservation) as a constraint on the MBO scheme and on Allen-Cahn flow arises in a number of contexts, which we shall here briefly survey. For a wider survey of general MBO schemes and Allen-Cahn flow in both the continuum and graph contexts, see [38] and the references therein.

In the continuum context, mass-conserving dynamics of the Ginzburg-Landau energy have a long history, dating back to [16] and [17] and the development of the Cahn-Hilliard equation. In the 1990s, Rubinstein and Sternberg [34] devised a mass-conserving variant of the Allen-Cahn equation as an alternative to the Cahn-Hilliard equation. More recently, Chen et al. [19] rigorously proved that mass-conserving mean curvature flow is the phase field limit of Rubinstein and Sternberg's mass-conserving Allen-Cahn flow. We will use Rubinstein and Sternberg's equation as the basis for our mass-conserving graph Allen-Cahn equation.

Just as the original MBO scheme was introduced as a method for mean curvature flow in Merriman et al. [5], mass-constrained MBO schemes in the continuum have been investigated as methods for studying mass-constrained mean curvature flow. It was first introduced as such in Ruuth and Wetton [36], and the convergence of this scheme has been recently studied by Laux and Schwartz [28], who showed that as the time step goes to zero, the algorithm of Ruuth and Wetton converges (up to a subsequence) to the weak formulation of mass-constrained mean curvature flow defined in [33].

The links between the non-mass-conserving Allen-Cahn flow and MBO scheme in the continuum are well-studied, as both have deep links to mean curvature flow (see e.g. [13] for details on the convergence of Allen-Cahn flow to mean curvature flow, and [22] for details on the convergence of the MBO scheme). As mentioned above, [19] and [28] show that the same links (now via mass-conserving mean curvature flow) hold in the mass-conserving case. We will demonstrate that this link between the mass-conserving Allen-Cahn flow and MBO scheme translates into the graph context.

Turning to the graph context, recently Van Gennip [37] studied a graph analogue of the Ohta-Kawasaki functional and devised a modified graph MBO scheme (with the ordinary MBO scheme as a special case) and mass-conserving graph MBO scheme as a method for minimising this functional without and with a mass conservation constraint, respectively. We will show that the mass-conserving MBO scheme yielded by applying the technique from [14] to the Rubinstein and Sternberg Allen-Cahn equation on a graph coincides with this definition of the mass-conserving MBO scheme on graphs (up to non-uniqueness of MBO solutions).

Finally, graph Allen-Cahn flow and MBO schemes have received much attention in the last decade as algorithms for image processing and semi-supervised learning, stemming from pioneering work by Bertozzi and Flenner [6] and Merkurjev et al. [31], respectively, and extended to the multi-class case in Merkurjev et al. [30]. Bae and Merkurjev [3] studied the effect of mass conservation constraints on these algorithms, inspiring Jacobs, Merkurjev, and Esedoḡu [26] to employ 'auction dynamics' as a novel way to solve a mass-conserving multi-class graph MBO scheme. In this work, we extend the link developed in [14] between these image-processing algorithms to this mass-conserving setting in the two-class case and demonstrate how to define our framework in the multi-class case. In future work, we seek to extend the theory of this paper to the multi-class case, and so link up with this body of work. 


\section{Groundwork}

We here rewrite the abridged summary of [38] from [14]. We henceforth consider weighted graphs $G:=(V, E, \omega)$ which are finite, simple, connected, undirected, and positively weighted, with vertex set $V$, edge set $E \subseteq V^{2}$, and with weights $\left\{\omega_{i j}\right\}_{i j \in E}$ satisfying $\omega_{i j}=\omega_{j i}$ and $\omega_{i j} \geqslant 0$ for all $i j \in E$. We extend $\omega_{i j}=0$ when $i j \notin E$. We define function spaces on $G$ (where $X \subseteq \mathbb{R}$, and $T \subseteq \mathbb{R}$ an interval):

$$
\begin{aligned}
& \mathcal{V}:=\{u: V \rightarrow \mathbb{R}\}, \quad \mathcal{V}_{X}:=\{u: V \rightarrow X\}, \quad \mathcal{E}:=\{\varphi: E \rightarrow \mathbb{R}\} . \\
& \mathcal{V}_{t \in T}:=\{u: T \rightarrow \mathcal{V}\}, \quad \mathcal{V}_{X, t \in T}:=\left\{u: T \rightarrow \mathcal{V}_{X}\right\} \text {. }
\end{aligned}
$$

We introduce a Hilbert space structure on these function spaces. For $r \in[0,1]$, and writing $d_{i}:=$ $\sum_{j} \omega_{i j}$ for the degree of vertex $i$, we define inner products on $\mathcal{V}$ and $\mathcal{E}$

$$
\langle u, v\rangle_{\mathcal{V}}:=\sum_{i \in V} u_{i} v_{i} d_{i}^{r}, \quad\langle\varphi, \phi\rangle_{\mathcal{E}}:=\frac{1}{2} \sum_{i, j \in V} \varphi_{i j} \phi_{i j} \omega_{i j}
$$

and define the inner product on $\mathcal{V}_{t \in T}\left(\right.$ or $\left.\mathcal{V}_{X, t \in T}\right)$

$$
(u, v)_{t \in T}:=\int_{T}\langle u(t), v(t)\rangle_{\mathcal{V}} d t=\sum_{i \in V} d_{i}^{r}\left(u_{i}, v_{i}\right)_{L^{2}(T ; \mathbb{R})} .
$$

The parameter $r$ has two roles: First and foremost, when we later define the graph Laplacian, $r$ will parameterise the normalisation of the graph Laplacian. Second, it appears in $\langle\cdot, \cdot\rangle_{\mathcal{V}}$ to ensure that any such choice of graph Laplacian is self-adjoint with respect to $\mathcal{V}$. We then induce inner product norms $\|\cdot\| \mathcal{V},\|\cdot\|_{\mathcal{E}}$, and $\|\cdot\|_{t \in T}$ and also define on $\mathcal{V}$ the norm $\|u\|_{\infty}:=\max _{i \in V}\left|u_{i}\right|$. Next, we define the $L^{2}$ and $L^{\infty}$ spaces:

$$
\begin{aligned}
& L^{2}(T ; \mathcal{V}):=\left\{u \in \mathcal{V}_{t \in T} \mid\|u\|_{t \in T}<\infty\right\} \\
& L^{\infty}(T ; \mathcal{V}):=\left\{u \in \mathcal{V}_{t \in T} \mid \exists C \in \mathbb{R},\|u(t)\|_{\infty}<C \text { for a.e. } t \in T\right\}
\end{aligned}
$$

Finally, for $T$ an open interval, we define the Sobolev space $H^{1}(T ; \mathcal{V})$ as the set of $u \in L^{2}(T ; \mathcal{V})$ with weak derivative $d u / d t \in L^{2}(T ; \mathcal{V})$ such that

$$
\forall \varphi \in C_{c}^{\infty}(T ; \mathcal{V})\left(u, \frac{d \varphi}{d t}\right)_{t \in T}=-\left(\frac{d u}{d t}, \varphi\right)_{t \in T},
$$

where $C_{c}^{\infty}(T ; \mathcal{V})$ denotes the set of elements of $\mathcal{V}_{t \in T}$ that are infinitely differentiable with respect to time and compactly supported in $T$. By [14, Proposition 2.1], $u \in H^{1}(T ; \mathcal{V})$ if and only if $u_{i} \in H^{1}(T ; \mathbb{R})$ for each $i \in V$. Then, $H^{1}(T ; \mathcal{V})$ has inner product:

$$
(u, v)_{H^{1}(T ; \mathcal{V})}:=(u, v)_{t \in T}+\left(\frac{d u}{d t}, \frac{d v}{d t}\right)_{t \in T}=\sum_{i \in V} d_{i}^{r}\left(u_{i}, v_{i}\right)_{H^{1}(T ; \mathbb{R})} .
$$

We also define the local $H^{1}$ space on any interval $T$ :

$$
H_{l o c}^{1}(T ; \mathcal{V}):=\left\{u \in \mathcal{V}_{t \in T} \mid \forall a, b \in T, u \in H^{1}((a, b) ; \mathcal{V})\right\}
$$

and likewise define $L_{l o c}^{2}(T ; \mathcal{V})$ and $L_{l o c}^{\infty}(T ; \mathcal{V})$ 
We introduce some notation: for $A \subseteq V$, define $\chi_{A} \in \mathcal{V}$ by

$$
\left(\chi_{A}\right)_{i}:= \begin{cases}1, & \text { if } i \in A, \\ 0, & \text { if } i \notin A .\end{cases}
$$

Next, we introduce the graph gradient and Laplacian:

$$
(\nabla u)_{i j}:=\left\{\begin{array}{ll}
u_{j}-u_{i}, & i j \in E \\
0, & \text { otherwise }
\end{array} \quad(\Delta u)_{i}:=d_{i}^{-r} \sum_{j \in V} \omega_{i j}\left(u_{i}-u_{j}\right) .\right.
$$

We note that $\Delta$ is positive semi-definite and self-adjoint with respect to $\mathcal{V}$. As shown in [38, p. 7], these operators are related via:

$$
\langle u, \Delta v\rangle_{\mathcal{V}}=\langle\nabla u, \nabla v\rangle_{\mathcal{E}}
$$

From $\Delta$, we define the graph diffusion operator:

$$
e^{-t \Delta} u:=\sum_{n \geqslant 0} \frac{(-1)^{n} t^{n}}{n !} \Delta^{n} u
$$

where $v(t)=e^{-t \Delta} u$ is the unique solution to $d v / d t=-\Delta v$ with $v(0)=u$. Note that $e^{-t \Delta} \mathbf{1}=\mathbf{1}$, where $\mathbf{1}$ is the vector of ones, so graph diffusion is mass-conserving, i.e. $\left\langle e^{-t \Delta} u, \mathbf{1}\right\rangle_{\mathcal{V}}=\langle u, \mathbf{1}\rangle_{\mathcal{V}}$. By [14, Proposition 2.2], if $u \in H^{1}(T ; \mathcal{V})$ and $T$ is bounded below, then $e^{-t \Delta} u \in H^{1}(T ; \mathcal{V})$ with

$$
\frac{d}{d t}\left(e^{-t \Delta} u\right)=e^{-t \Delta} \frac{d u}{d t}-e^{-t \Delta} \Delta u
$$

We recall from functional analysis the notation, for any linear $F: \mathcal{V} \rightarrow \mathcal{V}$,

$$
\begin{aligned}
& \rho(F):=\max \{|\lambda|: \lambda \text { an eigenvalue of } F\} \\
& \|F\|:=\sup _{\|u\|_{\mathcal{V}=1}}\|F u\|_{\mathcal{V}}
\end{aligned}
$$

and recall the standard result that if $F$ is self-adjoint, then $\|F\|=\rho(F)$.

Finally, we recall the notation from [14, p. 4106]: for problems of the form

$$
\underset{x}{\operatorname{argmin}} f(x),
$$

we write $f \simeq g$ and say $f$ and $g$ are equivalent when $g(x)=a f(x)+b$ for $a>0$ and $b$ independent of $x$. As a result, replacing $f$ by $g$ does not affect the minimisers.

To define graph Allen-Cahn (AC) flow, we first define the graph Ginzburg-Landau functional as in [14, Definition 2.5] by

$$
\mathrm{GL}_{\varepsilon}(u):=\frac{1}{2}\|\nabla u\|_{\mathcal{E}}^{2}+\frac{1}{\varepsilon}\langle W \circ u, \mathbf{1}\rangle_{\mathcal{V}},
$$

where $W$ is a double-well potential and $\varepsilon>0$ is a scaling parameter. AC flow is then the $\langle\cdot, \cdot\rangle_{\mathcal{V}}$ gradient flow of $\mathrm{GL}_{\varepsilon}$, which for $W$ differentiable is given by the ODE

$$
\frac{d u}{d t}=-\Delta u-\frac{1}{\varepsilon} W^{\prime} \circ u=-\nabla_{\mathcal{V}} \mathrm{GL}_{\varepsilon}(u),
$$

where $\nabla_{\mathcal{V}}$ is the Hilbert space gradient on $\mathcal{V}$. 
In [14, (2.5)], AC flow was linked to the MBO scheme via a discretisation of it by the 'semidiscrete' implicit Euler scheme (with time step $\tau \geqslant 0$ ):

$$
u_{n+1}=e^{-\tau \Delta} u_{n}-\frac{\tau}{\varepsilon} W^{\prime} \circ u_{n+1} .
$$

The name 'semi-discrete' refers to the fact that the scheme uses the exact solution operator for the diffusion part of the AC ODE and uses an implicit Euler time discretisation for the potential term. Equation (2.4) can be interpreted as an Euler scheme for a time-splitting scheme for AC flow, as was detailed in [14, Section 4.3] in the non-mass-conserving case. This scheme obeys the variational equation:

$$
u_{n+1} \in \underset{u \in \mathcal{V}}{\operatorname{argmin}} \frac{1}{\varepsilon}\langle W \circ u, \mathbf{1}\rangle_{\mathcal{V}}+\frac{\left\|u-e^{-\tau \Delta} u_{n}\right\|_{\mathcal{V}}^{2}}{2 \tau} .
$$

We now define the MBO scheme.

Definition 2.1 (Mass-conserving graph MBO scheme) We define the mass-conserving graph Merriman-Bence-Osher (MBO) scheme by the sequence of variational problems:

$$
u_{n+1} \in \underset{\substack{u \in \mathcal{V}_{[0,1]} \\\langle u, \mathbf{1}\rangle}=\left\langle u_{n}, \mathbf{1}\right\rangle_{\mathcal{V}}}{\operatorname{argmin}}\left\langle\mathbf{1}-2 e^{-\tau \Delta} u_{n}, u\right\rangle_{\mathcal{V}} .
$$

This is motivated by recalling the result from [38, Proposition 4.6] that the ordinary graph MBO scheme, defined as an iterative diffusion (for a time $\tau$ ) and thresholding scheme, is equivalent to the sequence of variational problems:

$$
u_{n+1} \in \underset{u \in \mathcal{V}_{[0,1]}}{\operatorname{argmin}}\left\langle\mathbf{1}-2 e^{-\tau \Delta} u_{n}, u\right\rangle_{\mathcal{V}}
$$

to which we have added a mass conservation constraint on the minimiser. Note that we can suppress the now constant $\langle\mathbf{1}, u\rangle_{\mathcal{V}}$ term.

To link the AC flow to the MBO scheme, as in [14], we take as $W$ the double-obstacle potential:

$$
W(x):= \begin{cases}\frac{1}{2} x(1-x), & \text { for } 0 \leqslant x \leqslant 1 \\ \infty, & \text { otherwise. }\end{cases}
$$

See also Blowey and Elliott [7, 8, 9] for study of this potential in the continuum context and Bosch et al. [10] for recent work in the graph context.

This choice of potential is essential to the whole of this work, so to motivate this choice, we review some of its virtues. One of the key advantages of this potential over smooth alternatives (as previously noted by Chen and Elliot in [18, p. 430], where a bunch of other more continuum-centric virtues are also discussed) is that it forces solutions to lie in $\mathcal{V}_{[0,1]}$ come-whatmay, whatever extra constraints or dynamics are imposed. This property is especially important when trying to link up with the MBO hard thresholding. The quadratic form of the potential between the wells is also very convenient for a number of reasons. First, it means that $W^{\prime}$ is an affine function on $(0,1)$, leading to the resulting AC flow (i.e. (2.7) below) being analysable 
using the tools for linear ODEs/differential inclusions. Second, we recall from [14, (2.2)] that the MBO scheme can be written as

$$
u_{n+1} \in \underset{u \in \mathcal{V}_{[0,1]}}{\operatorname{argmin}} \frac{1}{2}\langle\mathbf{1}-u, u\rangle_{\mathcal{V}}+\frac{1}{2}\left\|u-e^{-\tau \Delta} u_{n}\right\|_{\mathcal{V}}^{2}=\underset{u \in \mathcal{V}_{[0,1]}}{\operatorname{argmin}} W \circ u+\frac{1}{2}\left\|u-e^{-\tau \Delta} u_{n}\right\|_{\mathcal{V}}^{2}
$$

and thus the $\frac{1}{2} x(1-x)$ term in $W$ is exactly the term that arises naturally in the MBO scheme. Finally, the negative quadratic part of $W \circ u$ is cancelled out by the quadratic $\left\|u-e^{-\tau \Delta} u_{n}\right\|_{\mathcal{V}}^{2}$ term to give a convex energy, which will allow us to employ the tools of convex optimisation to study these variational problems.

As $W$ is not differentiable, the AC flow has to be redefined via the sub-differential of $W$. As in [14, Definition 3.4], we say that a pair $(u, \beta) \in \mathcal{V}_{[0,1], t \in T} \times \mathcal{V}_{t \in T}$ is a solution to double-obstacle AC flow for any interval $T$ when $u \in H_{l o c}^{1}(T ; \mathcal{V})$ and for a.e. $t \in T$ :

$$
\varepsilon \frac{d u(t)}{d t}+\varepsilon \Delta u(t)+\frac{1}{2} \mathbf{1}-u(t)=\beta(t), \quad \beta(t) \in \mathcal{B}(u(t)),
$$

where $\mathcal{B}(u)$ is the set (for $I_{[0,1]}(x):=0$ if $x \in[0,1]$ and $I_{[0,1]}(x):=\infty$ otherwise)

$$
\mathcal{B}(u):=\left\{\alpha \in \mathcal{V} \mid \forall i \in V, \alpha_{i} \in-\partial I_{[0,1]}\left(u_{i}\right)\right\} .
$$

That is, $\mathcal{B}(u)=\emptyset$ if $u \notin \mathcal{V}_{[0,1]}$, and for $u \in \mathcal{V}_{[0,1]}$, it is the set of $\beta \in \mathcal{V}$ such that

$$
\beta_{i} \in \begin{cases}{[0, \infty),} & u_{i}=0, \\ \{0\}, & 0<u_{i}<1, \\ (-\infty, 0], & u_{i}=1 .\end{cases}
$$

The semi-discrete scheme (2.4) thus becomes, where $\lambda:=\tau / \varepsilon$,

$$
(1-\lambda) u_{n+1}-e^{-\tau \Delta} u_{n}+\frac{\lambda}{2} \mathbf{1}=\lambda \beta_{n+1},
$$

where $\beta_{n+1} \in \mathcal{B}\left(u_{n+1}\right)$. Then, the key result of [14, Theorem 4.2] is the derivation of the MBO scheme from AC flow via the semi-discrete scheme, i.e. that for $\varepsilon=\tau$, the solutions to (2.9) obey the variational equation:

$$
u_{n+1} \in \underset{u \in \mathcal{V}_{[0,1]}}{\operatorname{argmin}}\langle u, \mathbf{1}-u\rangle_{\mathcal{V}}+\left\|u-e^{-\tau \Delta} u_{n}\right\|_{\mathcal{V}}^{2}
$$

and thus the solutions are MBO trajectories.

This paper will follow this method to derive the mass-conserving MBO scheme as a special case of a semi-discrete scheme for a mass-conserving double-obstacle AC flow.

\subsection{A comparison of the semi-discrete scheme and the minimising movements scheme for $\mathbf{G L}_{\varepsilon}$}

It is fruitful to compare the semi-discrete scheme to the minimising movements scheme for $\mathrm{GL}_{\varepsilon}$ (see [2, Chapter 2] for details on minimising movements). For a fixed time step $\tau>0$, this is defined by the variational scheme

$$
u_{n+1} \in \underset{u \in \mathcal{V}}{\operatorname{argmin}} \mathrm{GL}_{\varepsilon}(u)+\frac{1}{2 \tau}\left\|u-u_{n}\right\|_{\mathcal{V}}^{2}
$$


or equivalently, when $W$ is the double-obstacle potential,

$$
u_{n+1} \in \underset{u \in \mathcal{V}_{[0,1]}}{\operatorname{argmin}} \frac{1}{2}\langle u, \Delta u\rangle_{\mathcal{V}}+\frac{1}{2 \varepsilon}\langle u, \mathbf{1}-u\rangle_{\mathcal{V}}+\frac{1}{2 \tau}\left\|u-u_{n}\right\|_{\mathcal{V}}^{2} .
$$

As in $[2,(2.0 .2)]$, this scheme corresponds to the implicit Euler scheme

$$
\frac{u_{n+1}-u_{n}}{\tau}=-\nabla_{\mathcal{V}} \mathrm{GL}_{\varepsilon}\left(u_{n+1}\right)
$$

Handling the non-differentiability via the sub-differential as in (2.9), a short calculation yields the following expression for this scheme:

$$
(1-\lambda) u_{n+1}-(I+\alpha \tau \Delta)^{-1} u_{n}+\frac{\lambda}{2} \mathbf{1}=\lambda(I+\alpha \tau \Delta)^{-1} \beta_{n+1},
$$

where $\beta_{n+1} \in \mathcal{B}\left(u_{n+1}\right), I$ is the identity matrix, and $\alpha:=1 /(1-\lambda)$. It is straightforward to formally compare and contrast (2.9) and (2.10): the only differences are the $(I+\alpha \tau \Delta)^{-1}$ matrices in place of the $e^{-\tau \Delta}$ matrix on the left-hand side and in place of the identity matrix on the right-hand side.

For $\lambda \uparrow 1$, we can quickly see that (2.10) is not going to behave like the MBO scheme. The eigenvalues of $(I+\alpha \tau \Delta)^{-1}$ are $v_{k}:=\left(1+\alpha \tau \gamma_{k}\right)^{-1}$ where the $\gamma_{k}$ are the eigenvalues of $\Delta$. Recall that $\gamma_{0}=0$ and for $k>0, \gamma_{k}>0$. Therefore as $\lambda \uparrow 1$ (and hence $\alpha \rightarrow \infty$ ) for all $k>0, v_{k} \rightarrow$ 0 , whilst $\nu_{0}$ will remain 1 . Recall that the zeroth eigenvector of $\Delta$, and hence the eigenvector corresponding to $v_{0}$, is $\langle\mathbf{1}, \mathbf{1}\rangle_{\mathcal{V}}{ }^{-1} \mathbf{1}$. Hence, $(I+\alpha \tau \Delta)^{-1} \rightarrow Q$ where $Q u:=\frac{\langle u, \mathbf{1}\rangle_{\mathcal{V}}}{\langle\mathbf{1}, \mathbf{1}\rangle_{\mathcal{V}}} \mathbf{1}$ and so for $\lambda \uparrow 1$ (2.10) becomes

$$
\left(\frac{1}{2}-\frac{\left\langle u_{n}, \mathbf{1}\right\rangle_{\mathcal{V}}}{\langle\mathbf{1}, \mathbf{1}\rangle_{\mathcal{V}}}\right) \mathbf{1}=\frac{\left\langle\beta_{n+1}, \mathbf{1}\right\rangle_{\mathcal{V}}}{\langle\mathbf{1}, \mathbf{1}\rangle_{\mathcal{V}}} \mathbf{1}
$$

Thus, the requirement that $\beta_{n+1} \in \mathcal{B}\left(u_{n+1}\right)$ imposes almost no constraint on the values of $u_{n+1}$, entailing only that if the bracketed term on the left-hand side is negative, then there must be an $i \in V$ with $\left(\beta_{n+1}\right)_{i}<0$ and so $\left(u_{n+1}\right)_{i}=1$, and if the term is positive, then there must likewise be an $i \in V$ with $\left(u_{n+1}\right)_{i}=0$.

Finally, let us non-rigorously sketch the asymptotics of (2.10) as $\tau, \lambda \downarrow 0$ with $\varepsilon$ fixed. Solving the recurrence relation, the $n$th term of the sequence generated by $(2.10)$ is given by

$$
u_{n}=\frac{1}{2} \mathbf{1}+\alpha^{n}(I+\alpha \tau \Delta)^{-n}\left(u_{0}-\frac{1}{2} \mathbf{1}\right)+\lambda \sum_{r=1}^{n} \alpha^{n-r+1}(I+\alpha \tau \Delta)^{-(n-r+1)} \beta_{n} .
$$

Now, we note that up to $\mathcal{O}(\tau): \alpha^{n} \approx e^{n \lambda}$, and $(I+\alpha \tau \Delta)^{-k} \approx e^{-k \alpha \tau \Delta} \approx e^{-k \tau \Delta}$, and so

$$
u_{n}=\frac{1}{2} \mathbf{1}+e^{n \lambda} e^{-n \tau \Delta}\left(u_{0}-\frac{1}{2} \mathbf{1}\right)+\lambda \sum_{r=1}^{n} e^{(n-r+1) \lambda} e^{-(n-r+1) \tau \Delta} \beta_{n}+\mathcal{O}(\tau) .
$$

This is almost the same expression as for the asymptotics of the semi-discrete scheme derived in [14, Section 5], and thus the minimising movements scheme converges to the AC solution by roughly the same argument as given in that section. Indeed, note that by [14, Proposition 5.1], the semi-discrete scheme sequence has its $n$th term given by

$$
u_{n}=\frac{1}{2} \mathbf{1}+\alpha^{n} e^{-n \tau \Delta}\left(u_{0}-\frac{1}{2} \mathbf{1}\right)+\lambda \sum_{r=1}^{n} \alpha^{n-r+1} e^{-(n-r) \tau \Delta} \beta_{n} .
$$

It is beyond the scope of this paper to compare (2.11) and (2.12) further. 


\subsection{A note on our assumptions on $G$}

As stated above, in this paper, we will assume that our graph $G$ is finite, simple, connected, undirected, and positively weighted. In this subsection, we will briefly discuss the consequences of relaxing these conditions.

The case of $G$ an infinite graph is a substantial divergence from our framework, affecting a large number of definitions and results. To detail, these effects would take us well beyond the scope of this paper; as an example, see e.g. [24] for the subtleties of defining $\Delta$ in the infinite case.

If $G$ is non-simple, it must have multi-edges or self-loops. Multi-edges are essentially harmless for our framework, as they behave exactly like a single edge with weight equal to the sum of the weights of the multi-edges. If $G$ has self-loops, then let $G^{\prime}$ be the simple subgraph of $G$ without those self-loops. Then, as shown in [1], the unnormalised Laplacian $\Delta_{u}$ on $G$ (defined as in [1, (1)]) and the unnormalised Laplacian $\Delta_{u}^{\prime}$ on $G^{\prime}$ (defined as the $r=0$ case of (2.1)) are related by

$$
\Delta_{u}=\Delta_{u}^{\prime}+M
$$

where $M$ is a diagonal matrix with diagonal entries $M_{i i}:=\omega_{i i}$. Therefore, diffusion and AC flow on $G$ correspond, respectively, to the ODEs

$$
\frac{d v}{d t}=-\Delta_{u} v=-\Delta_{u}^{\prime} v-M v \quad \text { and } \quad \frac{d v}{d t}=-\Delta_{u}^{\prime} v-M v-\frac{1}{\varepsilon} W^{\prime} \circ v .
$$

These can be observed to be special cases of (respectively) fidelity forced diffusion and fidelity forced AC flow on $G^{\prime}$, as defined in [15, Definitions 2.1 and 2.6]. One of the major topics of that paper is the extension of our theoretical framework, e.g. [14, Theorem 4.2], to those fidelity forced (but non-mass-conserving) flows. Finally, the degree matrices $D$ and $D^{\prime}$ on $G$ and $G^{\prime}$ are related by $D=D^{\prime}+M$, so it follows that the normalised Laplacians $\Delta:=D^{-r} \Delta_{u}$ and $\Delta^{\prime}:=$ $D^{\prime-r} \Delta_{u}^{\prime}$ are related by

$$
\Delta=\left(I+M D^{-1}\right)^{-r} \Delta^{\prime}+\left(D^{\prime}+M\right)^{-r} M=: M_{1} \Delta^{\prime}+M_{2},
$$

where $M_{1}$ and $M_{2}$ are diagonal matrices, so diffusion with a normalised Laplacian on $G$ corresponds to a forced and rescaled diffusion on $G^{\prime}$, and similarly for AC flow.

If $G$ is disconnected, it is a simple matter to apply our framework to each connected component of $G$.

If $G$ is directed, then there are a number of different approaches to defining the Laplacian on a directed graph. For example, in [32, p. 6] and [39], the unnormalised Laplacian is defined by $\Delta_{u}=D-A$ where $A$ is the (directed) adjacency matrix and $D$ is the diagonal matrix of outdegrees. An alternative approach, found in [40], is as follows: given a directed graph $G=(V, E)$, define vertex sets $\mathcal{H}, \mathcal{A} \subseteq V$ where $\mathcal{H}$ are the vertices with positive out-degrees $d_{i}^{\text {out }}$ and $\mathcal{A}$ are the vertices with positive in-degrees $d_{i}^{\text {in }}$ (note that $\mathcal{H} \cap \mathcal{A}$ need not be empty). Then, define the $\operatorname{map} T:\left.\left.\mathcal{V}\right|_{\mathcal{A}} \rightarrow \mathcal{V}\right|_{\mathcal{H}}$ given by, for all $i \in \mathcal{H}$,

$$
(T u)_{i}=\sum_{j \in \mathcal{A}} \frac{\omega_{i j}}{\sqrt{d_{i}^{\text {out }} d_{j}^{\text {in }}}} u_{j}
$$

with adjoint $T^{*}:\left.\left.\mathcal{V}\right|_{\mathcal{H}} \rightarrow \mathcal{V}\right|_{\mathcal{A}}$ given by, for all $j \in \mathcal{A}$,

$$
\left(T^{*} u\right)_{j}=\sum_{i \in \mathcal{H}} \frac{\omega_{i j}}{\sqrt{d_{i}^{\text {out }} d_{j}^{i n}}} u_{i} .
$$


Next, extend $T$ and $T^{*}$ to $\mathcal{V}$ by setting $(T u)_{i}=0$ and $\left(T^{*} u\right)_{j}=0$ for $i \notin \mathcal{H}$ and $j \notin \mathcal{A}$, and then for $\gamma \in[0,1]$ define the Laplacian $\Delta_{\gamma}:=I-\gamma T^{*} T-(1-\gamma) T T^{*}$. A third approach can be found in [25, Section 2]. It is beyond the scope of this work to examine which of these definitions works best with our framework, and to what extent our framework can be extended to directed graphs.

Finally, if $G$ is a signed graph (i.e. $G$ has negative weights), then define $E^{+}:=\left\{i j \in E \mid \omega_{i j}>0\right\}$ and $E^{-}:=\left\{i j \in E \mid \omega_{i j}<0\right\}$ and thus define the positively weighted graphs $G^{+}:=\left(V, E^{+},\left.\omega\right|_{E^{+}}\right)$ and $G^{-}:=\left(V, E^{-},-\left.\omega\right|_{E^{-}}\right)$. It was shown in $[20,(39)]$ that the unnormalised Laplacian $\Delta_{u}$ on $G$ (defined as in $[20,(36)]$ ) can be decomposed as

$$
\Delta_{u}=\Delta_{u}^{+}+Q_{u}^{-}
$$

where $\Delta_{u}^{+}$is the unnormalised Laplacian on $G^{+}$and $Q_{u}^{-}$is the unnormalised signless Laplacian (see [21, 23] for details) on $G^{-}$, defined by

$$
\left(Q_{u}^{-} v\right)_{i}:=\sum_{j \in V \text { s.t. } i j \in E^{-}}\left(-\omega_{i j}\right)\left(v_{i}+v_{j}\right)
$$

The authors of [20] then go on to define an AC flow and MBO scheme on $G$ and apply this to a number of clustering problems. It is a topic for future research whether our framework can be extended to link AC flow and the MBO scheme on signed graphs.

\section{Mass-conserving AC flow}

In [34], Rubinstein and Sternberg define a mass-conserving Allen-Cahn flow (on a domain $\Omega$ ) as the non-local reaction-diffusion PDE, where $u: \Omega \rightarrow \mathbb{R}$,

$$
\frac{\partial u}{\partial t}=\Delta u-W^{\prime}(u)+\frac{1}{|\Omega|} \int_{\Omega} W^{\prime}(u) d x
$$

with Neumann boundary conditions. We can readily formulate this on a graph, noting the differing sign convention on $\Delta$ and introducing our scaling, as the ODE

$$
\frac{d u}{d t}=-\Delta u-\frac{1}{\varepsilon} W^{\prime} \circ u+\frac{1}{\varepsilon} \frac{\left\langle W^{\prime} \circ u, \mathbf{1}\right\rangle_{\mathcal{V}}}{\langle\mathbf{1}, \mathbf{1}\rangle_{\mathcal{V}}} \mathbf{1}
$$

Finally, as above in (2.7), we account for the non-differentiability of $W$ to arrive at:

$$
\varepsilon \frac{d u}{d t}+\varepsilon \Delta u(t)-u(t)+\frac{\langle u(t), \mathbf{1}\rangle_{\mathcal{V}}}{\langle\mathbf{1}, \mathbf{1}\rangle_{\mathcal{V}}} \mathbf{1}=\beta(t)-\frac{\langle\beta(t), \mathbf{1}\rangle_{\mathcal{V}}}{\langle\mathbf{1}, \mathbf{1}\rangle_{\mathcal{V}}} \mathbf{1}, \quad \beta(t) \in \mathcal{B}(u(t))
$$

We verify the mass conservation property for $u$ continuous and $H^{1}$. We first recall a standard fact about continuous representatives of $H^{1}$ functions.

Lemma 3.1 (See [14, Lemma 3.1]) If $u \in H_{\text {loc }}^{1}(T ; \mathcal{V}) \cap C^{0}(T ; \mathcal{V})$ or $u \in H_{\text {loc }}^{1}(T ; \mathbb{R}) \cap C^{0}(T ; \mathbb{R})$, then $u$ is locally absolutely continuous on $T$. It follows that $u$ is differentiable a.e. in $T$, and the weak derivative equals the classical derivative a.e. in T.

Definition 3.2 Define the mass of $u \in \mathcal{V}$ to be

$$
\mathcal{M}(u):=\langle u, \mathbf{1}\rangle_{\mathcal{V}}
$$


Furthermore, define the average value of $u \in \mathcal{V}$ to be

$$
\bar{u}:=\frac{\mathcal{M}(u)}{\mathcal{M}(\mathbf{1})}
$$

Proposition 3.3 For any interval $T$ and $u \in H_{l o c}^{1}(T ; \mathcal{V}) \cap C^{0}(T ; \mathcal{V})$, if u obeys (3.3) at a.e. $t \in T$, then for a.e. $t \in T$

$$
\frac{d}{d t} \mathcal{M}(u(t))=0
$$

and so $\mathcal{M}(u(t))$ is constant.

Proof First, note that $\mathcal{M}(u(t)) \in H_{\text {loc }}^{1}(T ; \mathbb{R}) \cap C^{0}(T ; \mathbb{R})$ with

$$
\frac{d}{d t} \mathcal{M}(u(t))=\left\langle\frac{d u}{d t}, \mathbf{1}\right\rangle_{\mathcal{V}}
$$

since for any $\varphi \in C_{c}^{\infty}(T ; \mathbb{R})$

$$
\begin{aligned}
\int_{T}\langle u(t), \mathbf{1}\rangle_{\mathcal{V}} \frac{d \varphi}{d t} d t & =\int_{T}\left\langle u(t), \frac{d \varphi}{d t} \mathbf{1}\right\rangle_{\mathcal{V}} d t \\
& =-\int_{T}\left\langle\frac{d u}{d t}, \varphi(t) \mathbf{1}\right\rangle_{\mathcal{V}} d t=-\int_{T}\left\langle\frac{d u}{d t}, \mathbf{1}\right\rangle_{\mathcal{V}} \varphi(t) d t
\end{aligned}
$$

Then, for almost every $t \in T$, taking the mass of both sides of (3.3):

$$
\varepsilon\left\langle\frac{d u}{d t}, \mathbf{1}\right\rangle_{\mathcal{V}}+\varepsilon\langle\Delta u(t), \mathbf{1}\rangle_{\mathcal{V}}-\langle u(t), \mathbf{1}\rangle_{\mathcal{V}}+\frac{\langle u(t), \mathbf{1}\rangle_{\mathcal{V}}}{\langle\mathbf{1}, \mathbf{1}\rangle_{\mathcal{V}}}\langle\mathbf{1}, \mathbf{1}\rangle_{\mathcal{V}}=\langle\beta(t), \mathbf{1}\rangle_{\mathcal{V}}-\frac{\langle\beta(t), \mathbf{1}\rangle_{\mathcal{V}}}{\langle\mathbf{1}, \mathbf{1}\rangle_{\mathcal{V}}}\langle\mathbf{1}, \mathbf{1}\rangle_{\mathcal{V}}
$$

So, most of the terms cancel, and we are left with

$$
\left\langle\frac{d u}{d t}, \mathbf{1}\right\rangle_{\mathcal{V}}=-\langle\Delta u(t), \mathbf{1}\rangle_{\mathcal{V}}=0
$$

with the final equality because $\Delta$ is self-adjoint and $\Delta \mathbf{1}=\mathbf{0}$. Then, by absolute continuity, we infer that $\mathcal{M}(u(t))$ is constant.

As with the ordinary Allen-Cahn flow, not all values in the sub-differential are attained in valid trajectories. We use Lemma 3.1 to characterise the validly attained $\beta$.

Theorem 3.4 (Cf. [14, Theorem 3.2]) Let $(u, \beta)$ obey (3.3) at a.e. $t \in T$, with $u \in H_{l o c}^{1}(T ; \mathcal{V}) \cap$ $C^{0}(T ; \mathcal{V}) \cap \mathcal{V}_{[0,1], t \in T}$. Then, for a.e. $t \in T$ and all $i \in V$, we have

$$
\beta_{i}(t)-\bar{\beta}(t)= \begin{cases}\bar{u}+\varepsilon(\Delta u(t))_{i}, & \text { if } u_{i}(t)=0, \\ -\bar{\beta}(t), & \text { if } u_{i}(t) \in(0,1), \\ \bar{u}-1+\varepsilon(\Delta u(t))_{i}, & \text { if } u_{i}(t)=1 .\end{cases}
$$

Proof Since $\beta(t) \in \mathcal{B}(u(t))$ at a.e. $t \in T$, (3.6) holds at a.e. $t \in T$ for which $u_{i}(t) \in(0,1)$. Let $\tilde{T} \subseteq T$ denote the times when $u$ is differentiable and has classical derivative equal to its weak 
derivative. Since $u_{i}(t) \in[0,1]$ at all times, when $t \in \tilde{T}$ and $u_{i}(t) \in\{0,1\}$, we have $d u_{i} / d t=0$. Consider first $u_{i}(t)=0$. Then for a.e. such $t \in \tilde{T}$

$$
0=\varepsilon \frac{d u_{i}}{d t}(t)=-\varepsilon(\Delta u(t))_{i}+\beta_{i}(t)-\bar{\beta}(t)-\bar{u}
$$

so rearranging

$$
\beta_{i}(t)-\bar{\beta}(t)=\bar{u}+\varepsilon(\Delta u(t))_{i}
$$

Likewise for $u_{i}(t)=1$, we have for a.e. such $t \in \tilde{T}$

$$
\beta_{i}(t)-\bar{\beta}(t)=\bar{u}-1+\varepsilon(\Delta u(t))_{i}
$$

so (3.6) holds at a.e. $t \in \tilde{T}$. By Lemma 3.1, $T \backslash \tilde{T}$ is null, so (3.6) holds at a.e. $t \in T$.

Definition 3.5 (Mass-conserving double-obstacle AC flow) Let $T$ be any interval. A pair $(u, \beta) \in \mathcal{V}_{[0,1], t \in T} \times \mathcal{V}_{t \in T}$ is a solution to mass-conserving double-obstacle AC flow on $T$ when $u \in H_{l o c}^{1}(T ; \mathcal{V}) \cap C^{0}(T ; \mathcal{V})$ and for almost every $t \in T$

$$
\varepsilon \frac{d u}{d t}+\varepsilon \Delta u(t)-u(t)+\frac{\langle u(t), \mathbf{1}\rangle_{\mathcal{V}}}{\langle\mathbf{1}, \mathbf{1}\rangle_{\mathcal{V}}} \mathbf{1}=\beta(t)-\frac{\langle\beta(t), \mathbf{1}\rangle_{\mathcal{V}}}{\langle\mathbf{1}, \mathbf{1}\rangle_{\mathcal{V}}} \mathbf{1}, \quad \beta(t) \in \mathcal{B}(u(t))
$$

For brevity, we will often refer to just $u$ as a solution to (3.7) where we also understand (3.7) to inherit the conditions on $u$ (including the existence of a corresponding $\beta$ ).

\subsection{Weak form and explicit integral form}

In this section, we prove first a weak form of mass-conserving $\mathrm{AC}$ flow and then an explicit integral form. The weak form is not used in the remainder of this paper; however, it is of general interest as it is a mass-conserving graph analogue of the variational inequality form of the continuum double-obstacle AC flow (see e.g. [9, (1.16)]). In the continuum setting, that form played an important role in the analysis performed by Blowey and Elliott in [7, 8, 9]. The latter form we will later use to show the convergence of the semi-discrete scheme (Theorem 5.7).

Theorem 3.6 (Cf. [14, Theorem 3.8]) A function $u \in \mathcal{V}_{[0,1], t \in T} \cap H_{\text {loc }}^{1}(T ; \mathcal{V})$ (and associated $\beta$ ) is a solution to (3.7) if and only if for a.e. $t \in T$ and $\forall \eta \in \mathcal{V}_{[0,1]}$ such that $\mathcal{M}(\eta)=\mathcal{M}(u(t))$ (i.e. $\eta-u(t) \perp \mathbf{1})$, the following hold

$$
\begin{gathered}
\left\langle\varepsilon \frac{d u}{d t}-u(t), \eta-u(t)\right\rangle_{\mathcal{V}}+\varepsilon\langle\nabla u(t), \nabla \eta-\nabla u(t)\rangle_{\mathcal{E}} \geq 0 \\
\left\langle\frac{d u}{d t}, \mathbf{1}\right\rangle_{\mathcal{V}}=0
\end{gathered}
$$

Proof Let $u$ satisfy (3.7). Then for a.e. $t \in T$, we have (3.8b) and $\beta(t) \in \mathcal{B}(u(t))$, so in particular $\beta_{i}(t) \geqslant 0$ and $\beta_{i}(t) \leqslant 0$ when $u_{i}(t)$ is 0 and 1 , respectively. Therefore, for all $\eta \in \mathcal{V}_{[0,1]}$ with 
$\eta-u(t) \perp \mathbf{1}$, for a.e. $t \in T$, we verify (3.8a):

LHS

$$
\begin{aligned}
& =\left\langle-\varepsilon \Delta u(t)-\frac{\langle u(t), \mathbf{1}\rangle_{\mathcal{V}}}{\langle\mathbf{1}, \mathbf{1}\rangle_{\mathcal{V}}} \mathbf{1}+\beta(t)-\frac{\langle\beta(t), \mathbf{1}\rangle_{\mathcal{V}}}{\langle\mathbf{1}, \mathbf{1}\rangle_{\mathcal{V}}} \mathbf{1}, \eta-u(t)\right\rangle_{\mathcal{V}}+\varepsilon\langle\nabla u(t), \nabla \eta-\nabla u(t)\rangle_{\mathcal{E}} \\
& =\langle\beta(t), \eta-u(t)\rangle_{\mathcal{V}} \\
& =\sum_{\left\{i \mid u_{i}(t)=0\right\}} d_{i}^{r} \beta_{i}(t) \eta_{i}+\sum_{\left\{i \mid u_{i}(t)=1\right\}} d_{i}^{r} \beta_{i}(t)\left(\eta_{i}-1\right) \geqslant 0 .
\end{aligned}
$$

Now let $u \in \mathcal{V}_{[0,1], t \in T} \cap H_{\text {loc }}^{1}(T ; \mathcal{V})$ satisfy (3.8). Therefore by (3.8a), for a.e. $t \in T$ and all $\eta \in \mathcal{V}_{[0,1]}$ with $\eta-u(t) \perp \mathbf{1}$

$$
\left\langle\varepsilon \frac{d u}{d t}-u(t)+\varepsilon \Delta u(t), \eta-u(t)\right\rangle_{\mathcal{V}} \geq 0
$$

and so for any $\theta: T \rightarrow \mathbb{R}$, a.e. $t \in T$, and any $\eta$ as before,

$$
\left\langle\varepsilon \frac{d u}{d t}-u(t)+\varepsilon \Delta u(t)+\frac{\langle u(t), \mathbf{1}\rangle_{\mathcal{V}}}{\langle\mathbf{1}, \mathbf{1}\rangle_{\mathcal{V}}} \mathbf{1}+\theta(t) \mathbf{1}, \eta-u(t)\right\rangle_{\mathcal{V}} \geq 0
$$

Let $t \in T$ be any such $t$. For a specific $\theta$ to be determined later, define

$$
\beta(t):=\varepsilon \frac{d u}{d t}-u(t)+\varepsilon \Delta u(t)+\frac{\langle u(t), \mathbf{1}\rangle_{\mathcal{V}}}{\langle\mathbf{1}, \mathbf{1}\rangle_{\mathcal{V}}} \mathbf{1}+\theta(t) \mathbf{1} .
$$

By considering certain valid test functions $\eta$ for (3.9), we will show that $\theta(t)$ can be chosen so that $\beta(t) \in \mathcal{B}(u(t))$. Towards this, for any $i, j \in V$ and $v \in \mathcal{V}$, we define the set

$$
\Xi_{i, j, v}:=\left\{\xi \in \mathcal{V} \mid \forall k \notin\{i, j\}, \xi_{k}=0, \forall k \in\{i, j\}, \xi_{k} \in\left[-v_{k}, 1-v_{k}\right], \text { and } \mathcal{M}(\xi)=0\right\}
$$

which is constructed so that if $\xi \in \Xi_{i, j, u(t)}$, then $\eta:=u(t)+\xi$ is a valid test function. Hence for any $\xi \in \Xi_{i, j, u(t)}$, by (3.9) and (3.10), we have that

$$
d_{i}^{r} \xi_{i} \beta_{i}(t)+d_{j}^{r} \xi_{j} \beta_{j}(t) \geqslant 0
$$

and so, since $\mathcal{M}(\xi)=0$ (i.e. $d_{i}^{r} \xi_{i}+d_{j}^{r} \xi_{j}=0$ ), for any $\xi \in \Xi_{i, j, u(t)}$, we have that

$$
d_{i}^{r} \xi_{i}\left(\beta_{i}(t)-\beta_{j}(t)\right) \geqslant 0
$$

Note If $u_{i}(t)=0$ and $u_{j}(t)>0$, then for $0<\alpha \leqslant 1$ sufficiently small

$$
\xi_{j}=-\alpha u_{j}(t) \in\left[-u_{j}(t), 0\right) \quad \xi_{i}=\alpha d_{i}^{-r} d_{j}^{r} u_{j} \in\left(0,1-u_{i}(t)\right]
$$

is $a \xi \in \Xi_{i, j, u(t)}$ with $\xi_{i}>0$. Likewise, if $u_{i}(t)=1$ and $u_{j}(t)<1$, there is a $\xi \in \Xi_{i, j, u(t)}$ with $\xi_{i}<0$, and if $u_{i}(t), u_{j}(t) \in(0,1)$, there exist $\xi, \xi^{\prime} \in \Xi_{i, j, u(t)}$ with $\xi_{i}>0$ and $\xi_{i}^{\prime}<0$.

Next, first suppose $u_{j}(t) \in(0,1)$ for some $j \in V$. Then, we fix such a $j$ and choose $\theta(t)$ so that $\beta_{j}(t)=0$, and thus by (3.11) for any $i \in V$ and $\xi \in \Xi_{i, j, u(t)}$ :

$$
\xi_{i} \beta_{i}(t) \geqslant 0 \text {. }
$$


Then by the above note, if we choose a $\xi \in \Xi_{i, j, u(t)}$ with $\xi_{i}$ of the appropriate sign,

$$
\beta_{i}(t) \begin{cases}=0, & \text { if } u_{i}(t) \in(0,1), \\ \leqslant 0, & \text { if } u_{i}(t)=1, \\ \geqslant 0, & \text { if } u_{i}(t)=0\end{cases}
$$

and so $\beta(t) \in \mathcal{B}(u(t))$.

Next, suppose no such $j$ exists. By above if $u_{i}(t)=0$ and $u_{j}(t)=1$, then we can choose $\xi \in$ $\Xi_{i, j, u(t)}$ with $\xi_{i}>0$ and so by (3.11), we have that $\beta_{j}(t) \leqslant \beta_{i}(t)$. Thus, we can choose $\theta(t)$ to add an appropriate constant to the values of $\beta(t)$ so that

$$
0 \in\left[\max _{u_{j}(t)=1} \beta_{j}(t), \min _{u_{i}(t)=0} \beta_{i}(t)\right] .
$$

Hence, we have

$$
\beta_{i}(t) \begin{cases}\leqslant 0, & \text { if } u_{i}(t)=1 \\ \geqslant 0, & \text { if } u_{i}(t)=0\end{cases}
$$

so $\beta(t) \in \mathcal{B}(u(t))$. Therefore, we can choose $\theta$ so that $\beta(t) \in \mathcal{B}(u(t))$ at a.e. $t \in T$.

Note finally that whatever the choice of $\theta$, by (3.8b) and (3.10) we have at a.e. $t \in T$

$$
\langle\beta(t), \mathbf{1}\rangle_{\mathcal{V}}=\theta(t)\langle\mathbf{1}, \mathbf{1}\rangle_{\mathcal{V}}
$$

Hence by (3.10), at all such $t$

$$
\varepsilon \frac{d u}{d t}+\varepsilon \Delta u(t)-u(t)+\frac{\langle u(t), \mathbf{1}\rangle_{\mathcal{V}}}{\langle\mathbf{1}, \mathbf{1}\rangle_{\mathcal{V}}} \mathbf{1}=\beta(t)-\frac{\langle\beta(t), \mathbf{1}\rangle_{\mathcal{V}}}{\langle\mathbf{1}, \mathbf{1}\rangle_{\mathcal{V}}} \mathbf{1}
$$

and, by choice of $\theta(t), \beta(t) \in \mathcal{B}(u(t))$. Hence, $(u, \beta)$ solves (3.7).

Theorem 3.7 (Cf. [14, Theorem 3.7]) For $u \in \mathcal{V}_{[0,1], t \in T}$ and $\beta \in \mathcal{V}_{t \in T},(u, \beta)$ is a solution to (3.7) if and only if $\beta-\bar{\beta} \mathbf{1}$ is locally essentially bounded and locally integrable (where by 'locally' we mean on each bounded subinterval of $T), \beta(t) \in \mathcal{B}(u(t))$ for a.e. $t \in T$, and for all $t \in T$

$$
u(t)=\bar{u} \mathbf{1}+e^{t / \varepsilon} e^{-t \Delta}(u(0)-\bar{u} \mathbf{1})+\frac{1}{\varepsilon} e^{t / \varepsilon} e^{-t \Delta} \int_{0}^{t} e^{-s / \varepsilon} e^{s \Delta}(\beta(s)-\bar{\beta}(s) \mathbf{1}) d s .
$$

Proof Let $(u, \beta)$ solve (3.7). Then, $\beta-\bar{\beta} \mathbf{1}$ is a sum of a continuous function and the derivative of a $H_{l o c}^{1}$ function and hence is locally integrable. We shall prove that $\beta-\bar{\beta} \mathbf{1}$ is globally essentially bounded in Lemma 5.10. Finally, we rewrite (3.7) to obtain (3.12). Consider the expression:

$$
\varepsilon \frac{d}{d t}\left(e^{-t / \varepsilon} e^{t \Delta}(u-\bar{u} \mathbf{1})\right) .
$$

Applying the product rule, we obtain that for a.e. $t \in T$, 


$$
\begin{aligned}
\text { (3.13) } & =-e^{-t / \varepsilon} e^{t \Delta}(u-\bar{u} \mathbf{1})+\varepsilon e^{-t / \varepsilon} \frac{d}{d t}\left(e^{t \Delta}(u-\bar{u} \mathbf{1})\right) \\
& =-e^{-t / \varepsilon} e^{t \Delta}(u-\bar{u} \mathbf{1})+\varepsilon e^{-t / \varepsilon} e^{t \Delta} \Delta(u-\bar{u} \mathbf{1})+\varepsilon e^{-t / \varepsilon} e^{t \Delta} \frac{d u}{d t} \\
& =e^{-t / \varepsilon} e^{t \Delta}\left(\varepsilon \frac{d u}{d t}+\varepsilon \Delta u-u+\bar{u} \mathbf{1}\right)=e^{-t / \varepsilon} e^{t \Delta}\left(\beta(t)-\frac{\langle\beta(t), \mathbf{1}\rangle_{\mathcal{V}}}{\langle\mathbf{1}, \mathbf{1}\rangle_{\mathcal{V}}}\right)
\end{aligned}
$$

and therefore integrating both sides and applying the 'fundamental theorem of calculus' on $H^{1}$ [12, Theorem 8.2], we obtain the integral form.

Now let $\xi:=\beta-\bar{\beta} \mathbf{1}$ be locally essentially bounded and locally integrable, let $\beta(t) \in \mathcal{B}(u(t))$ for a.e. $t \in T$, and for all $t \in T$ let (3.12) hold. By differentiating and reversing the above steps, we get that $(u, \beta)$ obeys the ODE in (3.7) and, in particular, the weak derivative of $u$ is given by:

$$
\frac{d u}{d t}=\left(\frac{1}{\varepsilon} I-\Delta\right) e^{t / \varepsilon} e^{-t \Delta}(u(0)-\bar{u} \mathbf{1})+\frac{1}{\varepsilon} \xi(t)+\left(\frac{1}{\varepsilon} I-\Delta\right) \frac{1}{\varepsilon} \int_{0}^{t} e^{(t-s) / \varepsilon} e^{-(t-s) \Delta} \xi(s) d s .
$$

As $\xi$ is locally essentially bounded, by (3.12) $u$ is continuous, and since $u$ is bounded, it is locally $L^{2}$. Finally, by above, $d u / d t$ is a sum of (respectively) a smooth function, a locally essentially bounded function and the integral of a locally essentially bounded function, so is locally essentially bounded and hence locally $L^{2}$. Hence, $u \in H_{l o c}^{1}(T ; \mathcal{V})$.

Note The forward reference to Lemma 5.10 does not introduce circularity here because we do not use this aspect of the forward direction of this theorem until after proving that lemma. We will however use the converse direction in proving the convergence of the semi-discrete scheme (Theorem 5.7).

Note $B y(3.12)$, if $\beta(t)=\mathbf{0}$ for a.e. $t \in[0, \infty)$, then

$$
u(t)=\bar{u} \mathbf{1}+\sum_{k=1}^{|V|-1} e^{\left(1 / \varepsilon-\gamma_{k}\right) t}\left\langle u(0), \xi_{k}\right\rangle \mathcal{V} \xi_{k}
$$

where $\left\{\left(\gamma_{k}, \xi_{k}\right)\right\}_{k=0}^{|V|-1}$ are the orthonormal eigenpairs of $\Delta$ in increasing order of eigenvalue (so $\gamma_{0}=0$ and $\left.\xi_{0} \propto 1\right)$. Let $\ell$ be the least $k \geqslant 1$, such that $\left\langle u(0), \xi_{k}\right\rangle_{\mathcal{V}} \neq 0$. Then to leading order

$$
u(t) \approx \bar{u} \mathbf{1}+e^{\left(1 / \varepsilon-\gamma_{\ell}\right) t}\left\langle u(0), \xi_{\ell}\right\rangle_{\mathcal{V}} \xi_{\ell}
$$

which if $\gamma_{\ell}<1 / \varepsilon$ contradicts $u(t) \in \mathcal{V}_{[0,1]}$ for sufficiently large $t$. Hence, in such a case, we must have $\beta(t) \neq \mathbf{0}$ for a non-null subset of the time. In particular, if $\varepsilon<1 /\|\Delta\|$, then this holds unless $u(0)=\bar{u} \mathbf{1}$.

\subsection{Existence and uniqueness}

Finally, we have the following existence and uniqueness theory for (3.7). As was brought to our attention by one of the reviewers, these results also follow from standard gradient flow theory (see [2, Chapter 4 especially Theorem 4.0.4] for details; to apply this theory in the present case, it is important to note that $\mathrm{GL}_{\varepsilon}$ is proper, coercive, lower semicontinuous, and is $(-1)$-convex, and hence [2, (4.0.1) and Assumption 4.0.1] is satisfied). However, these techniques are more 
theoretically involved than are needed in the present case, so we will here present these results with more elementary proofs.

Theorem 3.8 Let $(u, \beta),(v, \gamma)$ solve (3.7) on $T:=\left[0, T_{0}\right]$ or $[0, \infty)$, with $u(0)=v(0)$. Then, for all $t \in T, u(t)=v(t)$, and there exists $\tilde{T}$ such that $T \backslash \tilde{T}$ has zero measure and for all $t \in \tilde{T}$, $\beta(t)-\gamma(t)=(\bar{\beta}(t)-\bar{\gamma}(t)) \mathbf{1}$. Furthermore, if $u_{i}(t) \in(0,1)$ for some $i \in V$ and $t \in \tilde{T}$, then $\beta(t)=$ $\gamma(t)$

Proof As $u$ and $v$ solve (3.7), by subtracting and since $\bar{u}=\bar{v}$ we get for a.e. $t \in T$

$$
\varepsilon \frac{d}{d t}(v(t)-u(t))+\varepsilon \Delta(v(t)-u(t))-(v(t)-u(t))=(\gamma(t)-\beta(t))+(\bar{\beta}(t)-\bar{\gamma}(t)) \mathbf{1} .
$$

Let $w:=v-u$ and take the inner product with $w$, noting that $\langle w, \mathbf{1}\rangle_{\mathcal{V}}=0$,

$$
\varepsilon\left\langle\frac{d w}{d t}, w(t)\right\rangle_{\mathcal{V}}+\varepsilon\langle\Delta w(t), w(t)\rangle_{\mathcal{V}}-\langle w(t), w(t)\rangle_{\mathcal{V}}=\langle\gamma(t)-\beta(t), w(t)\rangle_{\mathcal{V}}
$$

Consider $\left(v_{i}(t)-u_{i}(t)\right)\left(\gamma_{i}(t)-\beta_{i}(t)\right)$. If $v_{i}(t)=u_{i}(t)$, this equals 0 , if $v_{i}(t)>u_{i}(t)$ then a simple case check gives that therefore $\gamma_{i}(t) \leqslant \beta_{i}(t)$ and likewise if $v_{i}(t)<u_{i}(t)$, then $\gamma_{i}(t) \geqslant$ $\beta_{i}(t)$. Hence , $\langle\gamma(t)-\beta(t), w(t)\rangle_{\mathcal{V}} \leqslant 0$. Furthermore, since $\Delta$ is positive semi-definite, we have $\langle\Delta w(t), w(t)\rangle_{\mathcal{V}} \geqslant 0$. Therefore, by the above, we have for a.e. $t \in T$,

$$
\frac{1}{2} \varepsilon \frac{d}{d t}\|w(t)\|_{\mathcal{V}}^{2} \leqslant\|w(t)\|_{\mathcal{V}}^{2}
$$

and note that $w(0)=\mathbf{0}$. Hence, by Grönwall's differential inequality, we have that for all $t \in T$, $\|w(t)\|_{\mathcal{V}}^{2} \leqslant 0$. Therefore, for all $t \in T, v(t)=u(t)$.

Finally by Theorem 3.4, since $u=v$ on $T$, at a.e. $t \in T$ (in particular, at $t \in \tilde{T}$ for some $\tilde{T} \subseteq T$ with $T \backslash \tilde{T}$ of zero measure):

$$
\beta_{i}(t)-\gamma_{i}(t)= \begin{cases}\bar{\beta}(t)-\bar{\gamma}(t), & \text { if } u_{i}(t)=0, \\ 0, & \text { if } u_{i}(t) \in(0,1), \\ \bar{\beta}(t)-\bar{\gamma}(t), & \text { if } u_{i}(t)=1 .\end{cases}
$$

Therefore at $t \in \tilde{T}$, either $\beta(t)-\gamma(t)=(\bar{\beta}(t)-\bar{\gamma}(t)) \mathbf{1}$ or, if $u_{i}(t) \in(0,1)$ for some $i \in V$, then taking the average value of both sides, we get

$$
\bar{\beta}(t)-\bar{\gamma}(t)=(\bar{\beta}(t)-\bar{\gamma}(t)) \frac{\mathcal{M}\left(\chi_{\left\{i \mid u_{i}(t) \in\{0,1\}\right\}}\right)}{\mathcal{M}(\mathbf{1})}
$$

so $\bar{\beta}(t)-\bar{\gamma}(t)=0$ and hence $\beta(t)=\gamma(t)$.

Note There are only $2^{|V|}$ distinct $u$ such that $u_{i} \in\{0,1\}$ for all $i \in V$. Hence, if $\overline{u(0)} \in[0,1] \backslash$ $\left\{\bar{u} \mid u \in \mathcal{V}\right.$ and $\left.\forall i \in V, u_{i} \in\{0,1\}\right\}$, which is $[0,1]$ minus a finite set of points, then we must have $\beta(t)=\gamma(t)$ for a.e. $t \in T($ since $\overline{u(t)}=\overline{u(0)})$.

Theorem 3.9 Let $T=[0, \infty)$. Then, for all $u_{0} \in \mathcal{V}_{[0,1]}$, there exists $(u, \beta) \in \mathcal{V}_{[0,1], t \in T} \times \mathcal{V}_{t \in T}$ satisfying (3.7) with $u \in H_{\text {loc }}^{1}(T ; \mathcal{V}) \cap C^{0}(T ; \mathcal{V})$ and with $u(0)=u_{0}$ 
Proof We prove this as Theorem 5.7, by taking the limit as $\tau \downarrow 0$ of the semi-discrete approximations defined in (4.1). (We avoid circularity as we do not use this theorem until after we have proved Theorem 5.7.)

\subsection{A note on pinning}

It was observed in [38, Theorem 5.3] (for the standard quartic potential) and [14, Remark 4.7] (for the double-obstacle potential) that if $\varepsilon$ is too small, the AC flow 'pins' or 'freezes', i.e. any vertex initially in a well remains in that well for all time. We now show that a similar result holds true in the mass-conserving case.

Theorem 3.10 Let $S \subseteq V$ and let $u(t):=\chi_{S}$ for all $t \in T$. Then, $u$ solves (3.7) if and only if $\varepsilon \max _{i \in S^{c}}\left|\left(\Delta \chi_{S}\right)_{i}\right| \leqslant 1-\varepsilon \max _{i \in S}\left|\left(\Delta \chi_{S}\right)_{i}\right|$, which always holds if $\varepsilon \leqslant \frac{1}{2}|| \Delta \chi_{S} \|_{\infty}^{-1}$.

Proof Note that $u(t):=\chi_{S}$ for all $t \in T$ satisfies $u \in H_{l o c}^{1}(T ; \mathcal{V}) \cap C^{0}(T ; \mathcal{V}) \cap \mathcal{V}_{[0,1], t \in T}$. Hence, by plugging it into (3.7), such a $u$ solves (3.7) if and only if there exists a $\beta \in \mathcal{V}_{t \in T}$ such that for a.e. $t \in T, \beta(t) \in \mathcal{B}\left(\chi_{S}\right)$ and

$$
\varepsilon \Delta \chi_{S}-\chi_{S}+\overline{\chi_{S}} \mathbf{1}=\beta(t)-\overline{\beta(t)} \mathbf{1} .
$$

In turn, this holds if and only if there exists a $\beta^{\prime} \in \mathcal{V}$ such that for all $i \in S, \beta_{i}^{\prime} \leqslant 0$, for all $i \in S^{c}$, $\beta_{i}^{\prime} \geqslant 0$, and $\varepsilon \Delta \chi_{S}-\chi_{S}+\overline{\chi_{S}} \mathbf{1}=\beta^{\prime}-\overline{\beta^{\prime}} \mathbf{1}$. Observe that for all $\theta \in \mathbb{R}, \beta^{\prime \prime}:=\varepsilon \Delta \chi_{S}-\chi_{S}+\theta \mathbf{1}$ satisfies the latter condition, and furthermore if some $\beta^{\prime \prime \prime}$ satisfies the latter condition, then $\beta^{\prime \prime}-\overline{\beta^{\prime \prime}} \mathbf{1}=\beta^{\prime \prime \prime}-\overline{\beta^{\prime \prime \prime}} \mathbf{1}$ and so $\beta^{\prime \prime \prime}=\beta^{\prime \prime}+\left(\overline{\beta^{\prime \prime \prime}}-\overline{\beta^{\prime \prime}}\right) \mathbf{1}$. Thus, all $\beta^{\prime}$ satisfying $\varepsilon \Delta \chi_{S}-\chi_{S}+$ $\overline{\chi_{S}} \mathbf{1}=\beta^{\prime}-\overline{\beta^{\prime}} \mathbf{1}$ are of the form $\beta^{\prime}=\varepsilon \Delta \chi_{S}-\chi_{S}+\theta \mathbf{1}$.

Hence, $u$ solves (3.7) if and only if there exists a $\theta \in \mathbb{R}$ such that for all $i \in S, \varepsilon\left(\Delta \chi_{S}\right)_{i}-$ $1+\theta \leqslant 0$, and for all $i \in S^{c}, \varepsilon\left(\Delta \chi_{S}\right)_{i}+\theta \geqslant 0$. Note that by the definition of $\Delta,\left(\Delta \chi_{S}\right)_{i} \geqslant 0$ for $i \in S$ and $\left(\Delta \chi_{S}\right)_{i} \leqslant 0$ for $i \in S^{c}$. Therefore, such a $\theta$ exists if and only if $\left[\varepsilon \max _{i \in S^{c}}\left|\left(\Delta \chi_{S}\right)_{i}\right|, 1-\right.$ $\left.\varepsilon \max _{i \in S}\left|\left(\Delta \chi_{S}\right)_{i}\right|\right]$ is non-empty. Finally, if $\varepsilon\left\|\Delta \chi_{S}\right\|_{\infty} \leqslant \frac{1}{2}$, then it suffices to take $\theta=\frac{1}{2}$.

\section{Mass-conserving semi-discrete scheme and link to the MBO scheme}

Definition 4.1 (Mass-conserving semi-discrete scheme) Building on the insight from [14], we link the mass-conserving AC flow to the mass-conserving MBO scheme by defining the following mass-conserving semi-discrete scheme:

$$
u_{n+1}-e^{-\tau \Delta} u_{n}-\lambda u_{n+1}+\lambda \frac{\left\langle u_{n+1}, \mathbf{1}\right\rangle_{\mathcal{V}}}{\langle\mathbf{1}, \mathbf{1}\rangle_{\mathcal{V}}} \mathbf{1}=\lambda \beta_{n+1}-\lambda \frac{\left\langle\beta_{n+1}, \mathbf{1}\right\rangle_{\mathcal{V}}}{\langle\mathbf{1}, \mathbf{1}\rangle_{\mathcal{V}}} \mathbf{1}
$$

for $\beta_{n+1} \in \mathcal{B}\left(u_{n+1}\right)$, recalling that $\lambda:=\tau / \varepsilon$. Recall that, by (2.8), since $\mathcal{B}\left(u_{n+1}\right)$ is non-empty, we must have $u_{n+1} \in \mathcal{V}_{[0,1]}$.

We check this conserves mass.

Proposition 4.2 For $u_{n+1}$ given by (4.1),

$$
\mathcal{M}\left(u_{n+1}\right)=\mathcal{M}\left(u_{n}\right) .
$$


Proof Taking the mass of both sides of (4.1) and cancelling gives

$$
\left\langle u_{n+1}, \mathbf{1}\right\rangle_{\mathcal{V}}=\left\langle e^{-\tau \Delta} u_{n}, \mathbf{1}\right\rangle_{\mathcal{V}}=\left\langle u_{n}, \mathbf{1}\right\rangle_{\mathcal{V}}
$$

with the final equality because $e^{-\tau \Delta}$ is self-adjoint and $e^{-\tau \Delta} \mathbf{1}=\mathbf{1}$.

We express this scheme variationally and link to the MBO scheme.

Theorem 4.3 (Cf. [14, Theorem 4.2]) If $0 \leqslant \tau \leqslant \varepsilon$, then the solutions to the semi-discrete scheme (4.1) obey

$$
\begin{aligned}
u_{n+1} \in \underset{\substack{u \in \mathcal{V}_{[0,1]} \\
\mathcal{M}(u)=\mathcal{M}\left(u_{n}\right)}}{\operatorname{argmin}} \lambda\langle u, \mathbf{1}-u\rangle_{\mathcal{V}}+\left\|u-e^{-\tau \Delta} u_{n}\right\|_{\mathcal{V}}^{2} \\
\simeq(1-\lambda)\|u\|_{\mathcal{V}}^{2}-2\left\langle u, e^{-\tau \Delta} u_{n}\right\rangle_{\mathcal{V}}
\end{aligned}
$$

In particular, when $\tau=\varepsilon$, we have

$$
u_{n+1} \in \underset{\substack{u \in \mathcal{V}_{[0,1]} \\ \mathcal{M}(u)=\mathcal{M}\left(u_{n}\right)}}{\operatorname{argmax}}\left\langle u, e^{-\tau \Delta} u_{n}\right\rangle_{\mathcal{V}}
$$

which is equivalent to the mass-conserving MBO scheme as in Definition 2.1.

Proof Let $u_{n+1}$ solve (4.1). First, note that $\mathcal{B}\left(u_{n+1}\right)$ is non-empty and so $u_{n+1} \in \mathcal{V}_{[0,1]}$. Furthermore, we know that $\mathcal{M}\left(u_{n+1}\right)=\mathcal{M}\left(u_{n}\right)=: M$.

Next, expanding out the functional for $\mathcal{M}(u)=M$ gives:

$$
\begin{aligned}
\lambda\langle u, \mathbf{1}-u\rangle_{\mathcal{V}}+\left\|u-e^{-\tau \Delta} u_{n}\right\|_{\mathcal{V}}^{2} & =\lambda M+(1-\lambda)\|u\|_{\mathcal{V}}^{2}-2\left\langle u, e^{-\tau \Delta} u_{n}\right\rangle_{\mathcal{V}}+\left\|e^{-\tau \Delta} u_{n}\right\|_{\mathcal{V}}^{2} \\
& \simeq(1-\lambda)\|u\|_{\mathcal{V}}^{2}-2\left\langle u, e^{-\tau \Delta} u_{n}\right\rangle_{\mathcal{V}} .
\end{aligned}
$$

We seek to prove that for $\lambda \leqslant 1$ and $\forall \eta \in \mathcal{V}_{[0,1]}$ such that $\langle\eta, \mathbf{1}\rangle_{\mathcal{V}}=M=\left\langle u_{n+1}, \mathbf{1}\right\rangle_{\mathcal{V}}$ :

$$
(1-\lambda)\left\langle u_{n+1}, u_{n+1}\right\rangle_{\mathcal{V}}-2\left\langle u_{n+1}, e^{-\tau \Delta} u_{n}\right\rangle_{\mathcal{V}} \leqslant(1-\lambda)\langle\eta, \eta\rangle_{\mathcal{V}}-2\left\langle\eta, e^{-\tau \Delta} u_{n}\right\rangle_{\mathcal{V}}
$$

By rearranging and cancelling, this is equivalent to (noting that $\left\langle\eta-u_{n+1}, \mathbf{1}\right\rangle_{\mathcal{V}}=0$ )

$$
\begin{aligned}
0 & \leqslant-\left\langle\eta-u_{n+1}, 2 e^{-\tau \Delta} u_{n}\right\rangle_{\mathcal{V}}+(1-\lambda)\left(\langle\eta, \eta\rangle_{\mathcal{V}}-\left\langle u_{n+1}, u_{n+1}\right\rangle_{\mathcal{V}}\right) \\
& =\left\langle\eta-u_{n+1},-2 e^{-\tau \Delta} u_{n}+(1-\lambda)\left(\eta+u_{n+1}\right)\right\rangle_{\mathcal{V}} \\
& =\left\langle\eta-u_{n+1}, 2(1-\lambda) u_{n+1}-2 e^{-\tau \Delta} u_{n}+(1-\lambda)\left(\eta-u_{n+1}\right)\right\rangle_{\mathcal{V}} \\
& =\left\langle\eta-u_{n+1}, 2 \lambda \beta_{n+1}-2 \lambda \overline{\beta_{n+1}} \mathbf{1}-2 \lambda \overline{u_{n+1}} \mathbf{1}+(1-\lambda)\left(\eta-u_{n+1}\right)\right\rangle_{\mathcal{V}} \quad \text { by }(4.1) \\
& =2 \lambda\left\langle\eta-u_{n+1}, \beta_{n+1}\right\rangle_{\mathcal{V}}+(1-\lambda)\left\|\eta-u_{n+1}\right\|_{\mathcal{V}}^{2}
\end{aligned}
$$

As $\beta_{n+1} \in \mathcal{B}\left(u_{n+1}\right)$ and $\eta_{i} \in[0,1]$ : either $\left(\beta_{n+1}\right)_{i}=0$, or $\left(\beta_{n+1}\right)_{i} \geqslant 0$ when $\eta_{i}-\left(u_{n+1}\right)_{i}=\eta_{i} \geqslant 0$, or $\left(\beta_{n+1}\right)_{i} \leqslant 0$ when $\eta_{i}-\left(u_{n+1}\right)_{i}=\eta_{i}-1 \leqslant 0$. Thus $\left\langle\eta-u_{n+1}, \beta_{n+1}\right\rangle_{\mathcal{V}} \geqslant 0$.

Finally, for $\lambda=1$, the quadratic term in (4.2) cancels and we get equation (4.3).

Note We briefly compare this behaviour to that of a mass-conserving minimising movements scheme for $\mathrm{GL}_{\varepsilon}$. This we define by

$$
u_{n+1} \in \underset{u \in \mathcal{V}, \mathcal{M}(u)=\mathcal{M}\left(u_{n}\right)}{\operatorname{argmin}} \operatorname{GL}_{\varepsilon}(u)+\frac{1}{2 \tau}\left\|u-u_{n}\right\|_{\mathcal{V}}^{2}
$$


or equivalently

$$
u_{n+1} \in \underset{u \in \mathcal{V}_{[0,1]}, \mathcal{M}(u)=\mathcal{M}\left(u_{n}\right)}{\operatorname{argmin}} \tau\langle u, \Delta u\rangle_{\mathcal{V}}-\lambda\|u\|_{\mathcal{V}}^{2}+\left\|u-u_{n}\right\|_{\mathcal{V}}^{2}
$$

For $\lambda \in[0,1)$, this is a strictly convex problem, so it has a unique solution which can be checked to obey

$$
(1-\lambda) u_{n+1}-(I+\alpha \tau \Delta)^{-1} u_{n}+\lambda \overline{u_{n}} \mathbf{1}=\lambda(I+\alpha \tau \Delta)^{-1} \beta_{n+1}-\lambda \overline{\beta_{n+1}} \mathbf{1},
$$

where $\beta_{n+1} \in \mathcal{B}\left(u_{n+1}\right)$ and $\alpha:=1 /(1-\lambda)$. This is an implicit Euler scheme for (3.7). One can then compare and contrast (4.4) with (4.1), which will follow similar lines as in the comparison for the ordinary case given in Section 2.1.

\subsection{Solving the variational equations}

Compared to the ordinary case, the addition of the mass conservation constraint substantially increases the difficulty in solving the equations from Theorem 4.3. We here employ the techniques of convex optimisation, particularly the Krein-Milman theorem, complementary slackness, and strong duality, to help resolve this difficulty.

We consider the set of feasible solutions to (4.2) and (4.3).

Definition 4.4 For a given $M=\mathcal{M}\left(u_{0}\right)$ for some $u_{0} \in \mathcal{V}_{[0,1]}$, we define the hyperplane $S_{M}:=$ $\left\{u \in \mathcal{V} \mid\langle u, \mathbf{1}\rangle_{\mathcal{V}}=M\right\}$. We can visualise this as the plane through $u_{0}$ with $\mathcal{V}$-normal vector $\mathbf{1}$. Then, we write the set of feasible solutions to (4.2) and (4.3)

$$
X:=\mathcal{V}_{[0,1]} \cap S_{M}
$$

Note that $X$ is compact and is the intersection of two convex sets, so is convex. Furthermore, note that $X$ can be described as the set of solutions to the linear inequalities

$$
\forall i \in V\left\langle u, \chi_{\{i\}}\right\rangle_{\mathcal{V}} \geqslant 0 \text { and }\left\langle u, \chi_{\{i\}}\right\rangle_{\mathcal{V}} \leqslant d_{i}^{r} \quad \text { and } \quad\langle u, \mathbf{1}\rangle_{\mathcal{V}} \geqslant M \text { and }\langle u, \mathbf{1}\rangle_{\mathcal{V}} \leqslant M
$$

and thus is said to be a polyhedral set.

Definition 4.5 For a convex set $C$, define $x \in C$ to be an extreme point of $C$ when

$$
\forall y, z \in C, \forall t \in(0,1) \quad(x=t y+(1-t) z \Rightarrow y=z=x)
$$

and write Ext $C$ for the subset of $C$ consisting of all such points.

We can then characterise the extreme points of the feasible set.

Proposition 4.6 The set $\operatorname{Ext} X$ of extreme points of $X$ is finite and is given by

$$
\operatorname{Ext} X=\left\{u \in X \mid \exists i^{*} \in V \forall j \in V \backslash\left\{i^{*}\right\} u_{j} \in\{0,1\}\right\} .
$$


Proof Since $X$ is polyhedral, Ext $X$ is finite by a standard result [27, Corollary 1.3.1]. Suppose $u \in X$ and $\exists i, j \in V$ such that $i \neq j$ and $u_{i}, u_{j} \in(0,1)$. Now for $\delta>0$ let

$$
\begin{aligned}
& v_{1}:=u-\delta d_{i}^{-r} \chi_{\{i\}}+\delta d_{j}^{-r} \chi_{\{j\}}, \\
& v_{2}:=u+\delta d_{i}^{-r} \chi_{\{i\}}-\delta d_{j}^{-r} \chi_{\{j\}} .
\end{aligned}
$$

Then, $\quad \mathcal{M}\left(v_{1}\right)=\mathcal{M}\left(v_{2}\right)=\mathcal{M}(u)-\delta+\delta=\mathcal{M}(u)=M \quad$ so $\quad v_{1}, v_{2} \in S_{M} . \quad$ And $\quad$ for $\quad \delta<$ $\min \left\{d_{i}^{r} u_{i}, d_{i}^{r}\left(1-u_{i}\right), d_{j}^{r} u_{j}, d_{j}^{r}\left(1-u_{j}\right)\right\}$, we have $v_{1}, v_{2} \in \mathcal{V}_{[0,1]}$. Therefore, we have $u=\frac{1}{2} v_{1}+\frac{1}{2} v_{2}$ for $v_{1}, v_{2} \in X \backslash\{u\}$. Therefore, $u \notin \operatorname{Ext} X$.

Now let $u \in\left\{u \in X \mid \exists i^{*} \in V \forall j \in V \backslash\left\{i^{*}\right\} u_{j} \in\{0,1\}\right\}$, and suppose $u=t v_{1}+(1-t) v_{2}$ for some $v_{1}, v_{2} \in X$ and $0<t<1$. As $\operatorname{Ext}([0,1])=\{0,1\}$, we have that $u_{i}=0$ if and only if $\left(v_{1}\right)_{i}=$ $\left(v_{2}\right)_{i}=0$ and likewise for $u_{i}=1$. So, $v_{1}-v_{2}=\theta \chi_{\left\{i^{*}\right\}}$ for some $\theta$, and

$$
0=\left\langle v_{1}-v_{2}, \mathbf{1}\right\rangle_{\mathcal{V}}=\theta\left\langle\chi_{\left\{i^{*}\right\}}, \mathbf{1}\right\rangle_{\mathcal{V}}=\theta d_{i^{*}}^{r}
$$

and so $\theta=0$, i.e. $v_{1}=v_{2}$. Thus, $u=t v_{1}+(1-t) v_{2} \Rightarrow v_{1}=v_{2}=u$, so $u \in \operatorname{Ext} X$.

For tidiness, we define some useful notation.

Definition 4.7 For $u \in \mathcal{V}_{[0,1]}$ and $\tau>0$ define the set

$$
A_{u, \tau}:=\left\{\alpha \in[0,1] \mid \exists i \in V\left(e^{-\tau \Delta} u\right)_{i}=\alpha\right\}
$$

with ordering $\alpha_{1}<\alpha_{2}<\ldots<\alpha_{K}$ for the elements of $A_{u, \tau}$, where $K=\left|A_{u, \tau}\right|$. Define the quantities

$$
a_{u, \tau, \alpha}:=\sum_{i:\left(e^{-\tau \Delta u}\right)_{i}=\alpha} d_{i}^{r} .
$$

Proposition 4.8 If $\tau>0$, then $0 \in A_{u, \tau} \Rightarrow u=\mathbf{0}$, and $1 \in A_{u, \tau} \Rightarrow u=\mathbf{1}$.

Proof Follows immediately from the connected graph case of [38, Lemma 2.6(d)].

\subsection{The MBO case: $\lambda=1$}

Definition 4.9 Define the set of solutions to (4.3)

$$
S_{\tau, u_{n}}:=\underset{u \in X}{\operatorname{argmax}}\left\langle u, e^{-\tau \Delta} u_{n}\right\rangle_{\mathcal{V}}
$$

This is convex as the objective function is linear and $X$ is convex, compact as it is a closed subset of $X$, and non-empty as $X$ is compact so the continuous objective function attains its maxima.

Proposition $4.10 S_{\tau, u_{n}}$ is a face of $X$, i.e. if $u, v \in X$ and $t \in(0,1)$, then

$$
t u+(1-t) v \in S_{\tau, u_{n}} \Rightarrow u, v \in S_{\tau, u_{n}} .
$$

Proof Let $u, v \in X, t \in(0,1)$, and $t u+(1-t) v \in S_{\tau, u_{n}}$. Then

$$
t\left\langle u, e^{-\tau \Delta} u_{n}\right\rangle_{\mathcal{V}}+(1-t)\left\langle v, e^{-\tau \Delta} u_{n}\right\rangle_{\mathcal{V}}=\max _{w \in X}\left\langle w, e^{-\tau \Delta} u_{n}\right\rangle_{\mathcal{V}}
$$


and so

$$
t\left\langle u, e^{-\tau \Delta} u_{n}\right\rangle_{\mathcal{V}} \geqslant \max _{w \in X}\left\langle w, e^{-\tau \Delta} u_{n}\right\rangle_{\mathcal{V}}-(1-t) \max _{w \in X}\left\langle w, e^{-\tau \Delta} u_{n}\right\rangle_{\mathcal{V}}=t \max _{w \in X}\left\langle w, e^{-\tau \Delta} u_{n}\right\rangle_{\mathcal{V}}
$$

and likewise for $\left\langle v, e^{-\tau \Delta} u_{n}\right\rangle_{\mathcal{V}}$. Hence,

$$
\left\langle u, e^{-\tau \Delta} u_{n}\right\rangle_{\mathcal{V}}=\left\langle v, e^{-\tau \Delta} u_{n}\right\rangle_{\mathcal{V}}=\max _{w \in X}\left\langle w, e^{-\tau \Delta} u_{n}\right\rangle_{\mathcal{V}},
$$

which is to say that $u, v \in S_{\tau, u_{n}}$.

Proposition 4.11 The extreme points of $S_{\tau, u_{n}}$ are given by

$$
\operatorname{Ext} S_{\tau, u_{n}}=S_{\tau, u_{n}} \cap \operatorname{Ext} X
$$

and the solutions to (4.3) are given by the convex hull of the extremal solutions, i.e.

$$
S_{\tau, u_{n}}=\operatorname{conv}\left(S_{\tau, u_{n}} \cap \operatorname{Ext} X\right) .
$$

Proof Let $u \in S_{\tau, u_{n}} \cap \operatorname{Ext} X$. Then, if $v_{1}, v_{2} \in S_{\tau, u_{n}} \subseteq X, t \in(0,1)$, and $u=t v_{1}+(1-t) v_{2}$, then $v_{1}=v_{2}$ since $u \in \operatorname{Ext} X$. So, $u \in \operatorname{Ext} S_{\tau, u_{n}}$.

Next, let $u \in \operatorname{Ext} S_{\tau, u_{n}} \subseteq S_{\tau, u_{n}}$. Then, if $v_{1}, v_{2} \in X$ and $u=t v_{1}+(1-t) v_{2}$, then $v_{1}, v_{2} \in S_{\tau, u_{n}}$ as $S_{\tau, u_{n}}$ is a face, and so $v_{1}=v_{2}$ since $u \in \operatorname{Ext} S_{\tau, u_{n}}$. Hence, $u \in S_{\tau, u_{n}} \cap \operatorname{Ext} X$.

So, Ext $S_{\tau, u_{n}}=S_{\tau, u_{n}} \cap \operatorname{Ext} X$, and finally we apply the Krein-Milman Theorem (see e.g. [35, 3.23]), which entails in particular that a finite-dimensional compact convex set is the convex hull of its extreme points.

Corollary 4.12 For $\mathcal{M}\left(u_{0}\right)=M$, there exists a trajectory $u_{n}$ obeying (4.3) such that

$$
\forall n \in \mathbb{N}, u_{n} \in \operatorname{Ext} X=\left\{u \in X \mid \exists i^{*} \in V \forall j \in V \backslash\left\{i^{*}\right\} u_{j} \in\{0,1\}\right\} .
$$

Proof Follows immediately from the fact that $S_{\tau, u_{n}}$ is non-empty, and so $S_{\tau, u_{n}} \cap \operatorname{Ext} X$ is nonempty as otherwise $S_{\tau, u_{n}}=\operatorname{conv}(\emptyset)=\emptyset$.

In [37, Section 5.3], Van Gennip considers a mass-conserving MBO scheme for minimising the Ohta-Kawasaki functional with a modified graph diffusion, which in the $\gamma=0$ special case reduces to ordinary graph diffusion and hence is the same problem as (4.3). We here repeat his form for the solutions to (4.3) lying at extreme points.

Theorem 4.13 Let $u_{n+1} \in S_{\tau, u_{n}} \cap \operatorname{Ext} X$. Then write

$$
E:=\left\{i \in V \mid\left(u_{n+1}\right)_{i}=1\right\}, F:=\left\{i \in V \mid\left(u_{n+1}\right)_{i}=0\right\}
$$

Then for each $i \in V \backslash F, j \in V \backslash E$ we have $\left(e^{-\tau \Delta} u_{n}\right)_{i} \geqslant\left(e^{-\tau \Delta} u_{n}\right)_{j}$.

Proof Recall that $u_{n+1}$ is a solution of (4.8). By Proposition 4.6, we have that $u_{n+1}=\chi_{E}+$ $\theta \chi_{V \backslash(E \cup F)}$ where $\theta \in(0,1)$ and $V \backslash(E \cup F)$ has at most one element which we will denote $i^{*}$ (when it exists). Now choose some $0<\delta<\min _{i \in V}\left\{d_{i}^{r}, d_{i^{*}}^{r} \theta, d_{i^{*}}^{r}(1-\theta)\right\}$, and any $i \in V \backslash F, j \in$ $V \backslash E$. Define

$$
u:=u_{n+1}-\delta d_{i}^{-r} \chi_{\{i\}}+\delta d_{j}^{-r} \chi_{\{j\}}
$$


where by choice of $\delta$, we ensure that $u \in X$. Therefore,

$$
0 \leqslant\left\langle u_{n+1}-u, e^{-\tau \Delta} u_{n}\right\rangle_{\mathcal{V}}=\delta\left(\left(e^{-\tau \Delta} u_{n}\right)_{i}-\left(e^{-\tau \Delta} u_{n}\right)_{j}\right)
$$

and so $\left(e^{-\tau \Delta} u_{n}\right)_{i} \geqslant\left(e^{-\tau \Delta} u_{n}\right)_{j}$ as desired.

\subsubsection{Uniqueness conditions for the mass-conserving MBO scheme}

We consider when (4.3) has a unique solution and characterise all solutions to (4.3).

Corollary 4.14 $S_{\tau, u_{n}}$ has one element if and only if $S_{\tau, u_{n}} \cap \operatorname{Ext} X$ has one element.

Proof As $S_{\tau, u_{n}}$ is non-empty, $S_{\tau, u_{n}} \cap \operatorname{Ext} X$ is non-empty as else $S_{\tau, u_{n}}=\operatorname{conv}(\emptyset)=\emptyset$. Thus, if $S_{\tau, u_{n}}=\{u\}$, then $S_{\tau, u_{n}} \cap \operatorname{Ext} X=\{u\}$ as this is the only non-empty subset of $S_{\tau, u_{n}}$. Conversely, if $S_{\tau, u_{n}} \cap \operatorname{Ext} X=\{u\}$, then by Proposition 4.11, $S_{\tau, u_{n}}=\operatorname{conv}(\{u\})=\{u\}$.

Usefully, Theorem 4.13 gives a necessary condition for $u \in S_{\tau, u_{n}} \cap \operatorname{Ext} X$. We demonstrate the following sufficient condition for uniqueness of solutions.

Theorem 4.15 Define the condition

$$
\forall i, j \in V, \quad i \neq j \Rightarrow\left(e^{-\tau \Delta} u_{n}\right)_{i} \neq\left(e^{-\tau \Delta} u_{n}\right)_{j} .
$$

Then if (4.9) holds, $S_{\tau, u_{n}}$ has a unique element (i.e. (4.3) has a unique solution).

Proof WLOG, up to relabelling of $V$, we may write (4.9) as

$$
i<j \Leftrightarrow\left(e^{-\tau \Delta} u_{n}\right)_{i}<\left(e^{-\tau \Delta} u_{n}\right)_{j} .
$$

Let $u \in S \cap \operatorname{Ext} X$. By Theorem 4.13, we thus have

$$
i<j \Rightarrow u_{i}=0 \text { or } u_{j}=1
$$

and hence by Proposition 4.6, $u$ must have the form

$$
u=(\underbrace{0,0, \ldots, 0}_{a-1}, \theta, \underbrace{1,1, \ldots, 1}_{|V|-a}),
$$

where $\theta \in(0,1]$ so $(a, \theta)$ uniquely determines any element of $S_{\tau, u_{n}} \cap \operatorname{Ext} X$. Let

$$
\mathcal{M}(a, \theta):=\mathcal{M}(u) \text { for } u \text { defined by }(a, \theta) \text { as above. }
$$

Then for $a<b$,

$$
\mathcal{M}(a, \theta)-\mathcal{M}(b, \phi)=\theta d_{a}^{r}+\sum_{a<i<b} d_{i}^{r}+(1-\phi) d_{b}^{r}>0
$$

and clearly $\mathcal{M}(a, \theta)=\mathcal{M}(a, \phi)$ if and only if $\theta=\phi$. If $u \in S_{\tau, u_{n}} \cap \operatorname{Ext} X, \mathcal{M}(u)=M$, and by the above, we have that $\mathcal{M}(a, \theta)=M$ for a unique $(a, \theta)$. Thus, $S_{\tau, u_{n}} \cap \operatorname{Ext} X$ has a unique element (as by the proof of Corollary 4.12, $S_{\tau, u_{n}} \cap \operatorname{Ext} X$ is non-empty), so by Corollary 4.14, $S_{\tau, u_{n}}$ has a unique element.

Following this idea, we get a characterisation of $S_{\tau, u_{n}}$ and a necessary and sufficient condition for uniqueness. 
Theorem 4.16 Suppose $u_{n} \in \mathcal{V}_{[0,1]}$ and $M=\mathcal{M}\left(u_{n}\right)>0$, then there is a unique $k$ such that $1 \leqslant$ $k \leqslant K$ and

$$
\sum_{\ell=k+1}^{K} a_{u_{n}, \tau, \alpha_{\ell}}<M \leqslant \sum_{\ell=k}^{K} a_{u_{n}, \tau, \alpha_{\ell}}
$$

recalling $K$ and $a_{u, \tau, \alpha}$ from Definition 4.7. Then, $u \in S_{\tau, u_{n}}$ if and only if $u \in X$ and

$$
\begin{aligned}
& u_{i}=0, \text { if }\left(e^{-\tau \Delta} u_{n}\right)_{i}<\alpha_{k}, \\
& u_{i}=1, \text { if }\left(e^{-\tau \Delta} u_{n}\right)_{i}>\alpha_{k}, \\
& M-\sum_{\ell=k+1}^{K} a_{u_{n}, \tau, \alpha_{\ell}}=\sum_{\left(e^{-\tau \Delta} u_{n}\right)_{i}=\alpha_{k}} d_{i}^{r} u_{i} .
\end{aligned}
$$

Therefore, $S_{\tau, u_{n}}$ has a unique element if and only if

$$
M=\sum_{\ell=k}^{K} a_{u_{n}, \tau, \alpha_{\ell}} \quad \text { or } \quad \exists ! i \in V,\left(e^{-\tau \Delta} u_{n}\right)_{i}=\alpha_{k} .
$$

Proof First, we show that $k$ exists and is unique. Let $B_{r}:=\sum_{\ell=r}^{K} a_{u_{n}, \tau, \alpha_{\ell}}$. Then as $a_{u_{n}, \tau, \alpha_{\ell}}>0$, the $B_{r}$ are strictly decreasing in $r$ and we observe that $B_{1}=\mathcal{M}(\mathbf{1}) \geqslant M$ and $B_{K+1}=0<M$. Hence, there exists a unique $k \in\{1, \ldots, K\}$ such that $B_{k+1}<M \leqslant B_{k}$.

Next, for $v \in \mathcal{V}$, define $\tilde{v}:\{1, \ldots, K\} \rightarrow \mathbb{R}$ by

$$
\tilde{v}_{\ell}:=a_{u_{n}, \tau, \alpha_{\ell}}^{-1} \sum_{i:\left(e^{-\tau \Delta} u_{n}\right)_{i}=\alpha_{\ell}} d_{i}^{r} v_{i}
$$

and define the inner product

$$
\langle\tilde{v}, \tilde{w}\rangle_{\alpha}:=\sum_{\ell=1}^{K} a_{u_{n}, \tau, \alpha_{\ell}} \tilde{v}_{\ell} \tilde{w}_{\ell}
$$

Then note by a simple calculation, we have that

$$
\langle\tilde{v}, \mathbf{1}\rangle_{\alpha}=\mathcal{M}(v)
$$

and

$$
\left\langle\tilde{v}, \widetilde{e^{-\tau \Delta} u_{n}}\right\rangle_{\alpha}=\left\langle v, e^{-\tau \Delta} u_{n}\right\rangle_{\mathcal{V}}
$$

Hence, defining $\tilde{X}=\{\tilde{v} \mid v \in X\}$, we have that $u \in S_{\tau, u_{n}}$ if and only if

$$
\tilde{u} \in \underset{\tilde{v} \in \tilde{X}}{\operatorname{argmax}}\left\langle\tilde{v}, \widetilde{e^{-\tau \Delta} u_{n}}\right\rangle_{\alpha}
$$

and note that (4.9) is satisfied by $\widetilde{e^{-\tau \Delta} u_{n}}$ (i.e. $\left(\widetilde{e^{-\tau \Delta} u_{n}}\right)_{\ell} \neq\left(\widetilde{e^{-\tau \Delta} u_{n}}\right)_{r}$ for all $\left.\ell \neq r \in\{1,2, \ldots, K\}\right)$. Therefore, by the same argument as in the proof of the previous theorem mutatis mutandis (i.e. replacing instances of $\langle\cdot, \cdot\rangle_{\mathcal{V}}$ with $\langle\cdot, \cdot\rangle_{\alpha}$, of $d_{i}^{r}$ with $a_{u_{n}, \tau, \alpha_{\ell}}$ etc.), there is a unique such $\tilde{u}$ of the form

$$
\tilde{u}=(\underbrace{0,0, \ldots, 0}_{b-1}, \theta, \underbrace{1,1, \ldots, 1}_{K-b})
$$


where $\theta \in(0,1]$. Then, we have

$$
M=\langle\tilde{u}, \mathbf{1}\rangle_{\alpha}=\theta a_{u_{n}, \tau, \alpha_{b}}+\sum_{\ell=b+1}^{K} a_{u_{n}, \tau, \alpha_{\ell}}
$$

so we must have $b=k$ and

$$
\theta=a_{u_{n}, \tau, \alpha_{k}}^{-1}\left(M-\sum_{\ell=k+1}^{K} a_{u_{n}, \tau, \alpha_{\ell}}\right) .
$$

Taking $\ell<k$,

$$
0=\tilde{u}_{\ell}=a_{u_{n}, \tau, \alpha_{\ell}}^{-1} \sum_{i:\left(e^{-\tau \Delta} u_{n}\right)_{i}=\alpha_{\ell}} d_{i}^{r} u_{i}
$$

and so $u_{i}=0$ if $\left(e^{-\tau \Delta} u_{n}\right)_{i}<\alpha_{k}$, and taking $\ell>k$

$$
1=\tilde{u}_{\ell}=a_{u_{n}, \tau, \alpha_{\ell}}^{-1} \sum_{i:\left(e^{-\tau \Delta} u_{n}\right)_{i}=\alpha_{\ell}} d_{i}^{r} u_{i}
$$

and so $u_{i}=1$ if $\left(e^{-\tau \Delta} u_{n}\right)_{i}>\alpha_{k}$. Finally taking $\ell=k$, we get the equivalences

$$
\begin{gathered}
u \in S_{\tau, u_{n}} \text { if and only if } \tilde{u} \in \underset{\tilde{v} \in \tilde{X}}{\operatorname{argmax}}\left\langle\tilde{v}, \widetilde{e^{-\tau \Delta} u_{n}}\right\rangle_{\alpha} \\
\text { if and only if }\left\{\begin{array}{l}
u_{i}=0, \text { if }\left(e^{-\tau \Delta} u_{n}\right)_{i}<\alpha_{k}, \\
u_{i}=1, \text { if }\left(e^{-\tau \Delta} u_{n}\right)_{i}>\alpha_{k}, \\
\theta=a_{u_{n}, \tau, \alpha_{k}}^{-1} \sum_{\left(e^{-\tau \Delta} u_{n}\right)_{i}=\alpha_{k}} d_{i}^{r} u_{i} .
\end{array}\right.
\end{gathered}
$$

Hence, we have a unique solution if and only if $\left(e^{-\tau \Delta} u_{n}\right)_{i}=\alpha_{k}$ at a unique $i \in V$ or $\theta=1$ (and therefore $u_{i}=1$ for $\left.\left(e^{-\tau \Delta} u_{n}\right)_{i}=\alpha_{k}\right)$, i.e. when (4.11) holds.

Note If $M=0$, then $X=\{\mathbf{0}\}$, so uniqueness is trivial, hence supposing that $M>0$ incurs no loss of generality.

Note The solution in (4.10), with an adjustable threshold level (i.e. $\alpha_{k}$ ) to ensure that mass is conserved, accords with the definition of the mass-conserving graph MBO scheme in [37] and with the definition of the mass-conserving continuum MBO scheme in [36]. We here note that there is a typo in the definition in [37] (i.e. [37, Algorithm (mcOKMBO)]): all instances of " $d_{i}^{r} u_{i}$ " in that definition should just read " $d_{i}^{r}$ ".

\subsection{The non-MBO case: $0 \leqslant \lambda<1$}

To solve (4.2) for $0 \leqslant \lambda<1$, we use duality. Let $M:=\mathcal{M}\left(u_{n}\right)$ and define the functions

$$
f_{i}(u):=-d_{i}^{r} u_{i}, \quad g_{i}(u):=\left(u_{i}-1\right) d_{i}^{r}, \quad h(u):=2(\mathcal{M}(u)-M) .
$$

Then, (4.2) can be written as the primal problem:

$$
\min _{u \in \mathcal{V}}(1-\lambda)\|u\|_{\mathcal{V}}^{2}-2\left\langle u, e^{-\tau \Delta} u_{n}\right\rangle_{\mathcal{V}} \text { s.t. } f_{i}(u) \leqslant 0, g_{i}(u) \leqslant 0 \text {, and } h(u)=0 .
$$


Hence, for $\xi, \mu \in \mathcal{V}$ and $v \in \mathbb{R}$ dual variables, (4.2) has Lagrangian:

$$
\begin{aligned}
& L(u, \xi, \mu, v):=(1-\lambda)\|u\|_{\mathcal{V}}^{2}-2\left\langle u, e^{-\tau \Delta} u_{n}\right\rangle_{\mathcal{V}}+\sum_{i}\left(\xi_{i} f_{i}(u)+\mu_{i} g_{i}(u)\right)+v h(u) \\
& =(1-\lambda)\|u\|_{\mathcal{V}}^{2}-2\left\langle u, e^{-\tau \Delta} u_{n}\right\rangle_{\mathcal{V}}+\langle u, \mu-\xi\rangle_{\mathcal{V}}+\langle 2 v u-\mu, \mathbf{1}\rangle_{\mathcal{V}}-2 v M
\end{aligned}
$$

We can rewrite this by making the following definition:

$$
u^{*}(\xi, \mu, \nu):=\frac{1}{2(1-\lambda)}\left(2 e^{-\tau \Delta} u_{n}+\xi-\mu-2 \nu \mathbf{1}\right)
$$

so that

$$
\begin{aligned}
L(u, \xi, \mu, \nu) & =(1-\lambda)\|u\|_{\mathcal{V}}^{2}-2(1-\lambda)\left\langle u, u^{*}(\xi, \mu, \nu)\right\rangle_{\mathcal{V}}-\langle\mu, \mathbf{1}\rangle_{\mathcal{V}}-2 v M \\
& =(1-\lambda)\left\|u-u^{*}(\xi, \mu, v)\right\|_{\mathcal{V}}^{2}-(1-\lambda)\left\|u^{*}(\xi, \mu, \nu)\right\|_{\mathcal{V}}^{2}-\langle\mu, \mathbf{1}\rangle_{\mathcal{V}}-2 v M,
\end{aligned}
$$

which we note is strictly convex, proper, and bounded below in $u$ (for fixed $\xi$, $\mu$, and $v$ ). Next, we define the dual objective function:

$$
G(\xi, \mu, v):=\inf _{u \in \mathcal{V}} L(u, \xi, \mu, v)=L\left(u^{*}(\xi, \mu, v), \xi, \mu, v\right)
$$

and therefore

$$
G(\xi, \mu, \nu)=-\left((1-\lambda)\left\|u^{*}(\xi, \mu, v)\right\|_{\mathcal{V}}^{2}+\langle\mu, \mathbf{1}\rangle_{\mathcal{V}}+2 v M\right)
$$

The dual problem to (4.2) is given by

$$
\sup _{\xi \geqslant 0, \mu \geqslant 0, v} G(\xi, \mu, \nu) .
$$

Lemma 4.17 For $u_{n} \in \mathcal{V}_{[0,1]}, M=\mathcal{M}\left(u_{n}\right)$, (4.2) and (4.17) have strong duality, i.e.

$$
\sup _{\xi \geqslant 0, \mu \geqslant 0, \nu} G(\xi, \mu, v)=\min _{u \in X}(1-\lambda)\|u\|_{\mathcal{V}}^{2}-2\left\langle u, e^{-\tau \Delta} u_{n}\right\rangle_{\mathcal{V}}
$$

and if $\xi^{*}, \mu^{*}$, and $v^{*}$ optimise (4.17), then $u^{*}:=u^{*}\left(\xi^{*}, \mu^{*}, v^{*}\right) \in X$ as in (4.14) optimises (4.2).

Proof We apply Slater's condition for strong duality (see [11, Section 5.2.3]). As the $f_{i}$ and $g_{i}$ are affine on $\mathcal{V}$ and $\mathcal{V}$ is open and affine, Slater's condition is satisfied if $\exists u \in \mathcal{V}$ with $f_{i}(u) \leqslant$ $0, g_{i}(u) \leqslant 0$, and $h(u)=0$, i.e. if $\exists u \in X$. As $u_{n} \in X$, we thus have strong duality.

Now let $\xi^{*} \geqslant 0, \mu^{*} \geqslant 0$, and $\nu^{*}$ be optimal for (4.17), and let $\hat{u} \in X$ be optimal for (4.2), which we know exists since $X$ is compact and the objective function is continuous. Writing $q(u):=$ $(1-\lambda)\|u\|_{\mathcal{V}}^{2}-2\left\langle u, e^{-\tau \Delta} u_{n}\right\rangle_{\mathcal{V}}$, we have by strong duality:

$$
q(\hat{u})=G\left(\xi^{*}, \mu^{*}, v^{*}\right)=L\left(u^{*}, \xi^{*}, \mu^{*}, v^{*}\right)=\inf _{u \in \mathcal{V}} L\left(u, \xi^{*}, \mu^{*}, v^{*}\right) \leqslant L\left(\hat{u}, \xi^{*}, \mu^{*}, v^{*}\right) \leqslant q(\hat{u}),
$$

where the final inequality holds by (4.13), as $\hat{u} \in X$ and so $f_{i}(\hat{u}), g_{i}(\hat{u}) \leqslant 0$, and $h(\hat{u})=0$. So, the inequalities are equalities and $L\left(u, \xi^{*}, \mu^{*}, v^{*}\right)$ is minimised at $\hat{u}$. As $L$ is strictly convex in $u$, it has a unique minimiser, so $u^{*}\left(\xi^{*}, \mu^{*}, v^{*}\right)=\hat{u}$ is optimal for (4.2). 
By Lemma 4.17, we have that $u^{*}:=u^{*}\left(\xi^{*}, \mu^{*}, v^{*}\right) \in X$ for $\left(\xi^{*}, \mu^{*}, v^{*}\right)$ dual optimal, and by applying complementary slackness, we have that

$$
\begin{aligned}
& u_{i}^{*}>0 \Rightarrow \xi_{i}^{*}=0 \text {, } \\
& \text { and } \\
& u_{i}^{*}<1 \Rightarrow \mu_{i}^{*}=0 \text {, } \\
& \xi_{i}^{*}>0 \Rightarrow u_{i}^{*}=0 \text {, } \\
& \mu_{i}^{*}>0 \Rightarrow u_{i}^{*}=1 \text {. }
\end{aligned}
$$

Thus, at each $i \in V, \xi_{i}^{*}=0$ or $\mu_{i}^{*}=0$. So, we have the necessary conditions

$$
u_{i}^{*}=\left\{\begin{array}{lll}
0 & \Rightarrow & \mu_{i}^{*}=0 \\
\in(0,1) & \Rightarrow & \xi_{i}^{*}=\mu_{i}^{*}=0 \\
1 & \Rightarrow & \xi_{i}^{*}=0
\end{array}\right.
$$

Then by substituting into (4.14)

$$
u_{i}^{*}=\left\{\begin{array}{cl}
0, & \text { if and only if } \mu_{i}^{*}=0, \xi_{i}^{*}=2 v^{*}-2\left(e^{-\tau \Delta} u_{n}\right)_{i} \geqslant 0, \\
\frac{\left(e^{-\tau \Delta} u_{n}\right)_{i}-v^{*}}{1-\lambda} & \text { if and only if } \xi_{i}^{*}=\mu_{i}^{*}=0,0<\left(e^{-\tau \Delta} u_{n}\right)_{i}-v^{*}<1-\lambda, \\
\in(0,1), & \text { if and only if } \xi_{i}^{*}=0, \mu_{i}^{*}=2\left(e^{-\tau \Delta} u_{n}\right)_{i}-2(1-\lambda)-2 v^{*} \geqslant 0 .
\end{array}\right.
$$

We simplify by noting that the $v^{*}$ inequality conditions are disjoint and exhaustive, so we need to only consider those conditions (to see this, note that if for example $v^{*} \geqslant\left(e^{-\tau \Delta} u_{n}\right)_{i}$, then each of the $u_{i}^{*}>0$ cases is ruled out, so $u_{i}^{*}$ must equal zero):

$$
u_{i}^{*}= \begin{cases}0, & \text { if and only if } v^{*}-\left(e^{-\tau \Delta} u_{n}\right)_{i} \geqslant 0, \\ \frac{\left(e^{-\tau \Delta} u_{n}\right)_{i}-v^{*}}{1-\lambda} \in(0,1), & \text { if and only if } 0<\left(e^{-\tau \Delta} u_{n}\right)_{i}-v^{*}<1-\lambda, \\ 1, & \text { if and only if } v^{*} \leqslant\left(e^{-\tau \Delta} u_{n}\right)_{i}-(1-\lambda) .\end{cases}
$$

But by the above lemma $u^{*} \in X$, so we have $\mathcal{M}\left(u^{*}\right)=M$. Thus, $v=v^{*}$ is a solution to:

$$
0=M+\sum_{i} d_{i}^{r} \begin{cases}-1, & v \leqslant\left(e^{-\tau \Delta} u_{n}\right)_{i}-(1-\lambda), \\ \frac{v-\left(e^{-\tau \Delta} u_{n}\right)_{i}}{1-\lambda}, & \left(e^{-\tau \Delta} u_{n}\right)_{i}-(1-\lambda)<v<\left(e^{-\tau \Delta} u_{n}\right)_{i}, \\ 0, & v \geqslant\left(e^{-\tau \Delta} u_{n}\right)_{i},\end{cases}
$$

which exists by the Intermediate Value Theorem. By Definition 4.7, we rewrite (4.19)

$$
M=\sum_{\alpha \in A_{u_{n}, \tau}} a_{u_{n}, \tau, \alpha} \begin{cases}1, & v \leqslant \alpha-(1-\lambda) \\ \frac{\alpha-\nu}{1-\lambda}, & \alpha-(1-\lambda)<v<\alpha \\ 0, & v \geqslant \alpha\end{cases}
$$

Note Although $u^{*}$ is unique, $v^{*}$ is not in general unique, but in such cases, each solution $v^{*}$ gives rise to the same $u^{*}$. For example, if $A_{u_{n}, \tau}=\{0\}$ (i.e. $u_{n}=\mathbf{0}$ ), then any $v \geqslant 0$ solves (4.20), but by the same token in that case, any $v \geqslant 0$ gives $u^{*}=\mathbf{0}$. In general, the right-hand side of (4.20) is 
constant in $v$ if and only if $v \in\left[\alpha_{k}, \alpha_{k+1}-(1-\lambda)\right]$, where $\alpha_{k}, \alpha_{k+1}$ are consecutive elements in $A_{u_{n}, \tau}$. But if $v$ in that interval solves (4.20), then by (4.18)

$$
u_{i}^{*}= \begin{cases}0, & \text { if and only if }\left(e^{-\tau \Delta} u_{n}\right)_{i} \leqslant \alpha_{k} \\ 1, & \text { if and only if }\left(e^{-\tau \Delta} u_{n}\right)_{i}>\alpha_{k}\end{cases}
$$

Finally, note that, therefore, this situation of non-unique $v^{*}$ can only arise if $M \in\{\mathcal{M}(u) \mid u \in$ $\left.\mathcal{V}_{\{0,1\}}\right\}$, which is a finite set of values.

Proposition 4.18 Let $u_{n} \in \mathcal{V}_{[0,1]}, M=\mathcal{M}\left(u_{n}\right)$, and suppose $0<M<\langle\mathbf{1}, \mathbf{1}\rangle_{\mathcal{V}}$ and $\tau>0$. If $v$ solves (4.20), then $v \in\left[\lambda \min A_{u_{n}, \tau}, \lambda \max A_{u_{n}, \tau}\right] \subseteq(0, \lambda)$.

Proof By Proposition 4.8 and the condition on $M$, note that $A_{u_{n}, \tau} \subseteq(0,1)$. Since diffusion preserves mass, $M=\mathcal{M}\left(e^{-\tau \Delta} u_{n}\right)$ and therefore

$$
M=\sum_{\alpha \in A_{u_{n}, \tau}} a_{u_{n}, \tau, \alpha} \alpha
$$

and so we have by (4.20):

$$
0=\sum_{\alpha \in A_{u_{n}, \tau}} a_{u_{n}, \tau, \alpha} \begin{cases}1-\alpha, & v \leqslant \alpha-(1-\lambda), \\ \frac{\alpha-\nu}{1-\lambda}-\alpha, & \alpha-(1-\lambda)<v<\alpha, \\ -\alpha, & v \geqslant \alpha,\end{cases}
$$

i.e., $v$ is a solution to

$$
\begin{aligned}
0=\sum_{\alpha \in[1-\lambda+v, 1) \cap A_{u_{n}, \tau}} a_{u_{n}, \tau, \alpha}(1-\alpha) & +\sum_{\alpha \in(v, 1-\lambda+v) \cap A_{u_{n}, \tau}} a_{u_{n}, \tau, \alpha} \frac{\alpha \lambda-v}{1-\lambda} \\
& +\sum_{\alpha \in(0, v] \cap A_{u_{n}, \tau}} a_{u_{n}, \tau, \alpha}(-\alpha) .
\end{aligned}
$$

First, suppose that $v<\lambda \min A_{u_{n}, \tau}<\min A_{u_{n}, \tau}$. Then

$$
\sum_{\alpha \in(0, v] \cap A_{u_{n}, \tau}} a_{u_{n}, \tau, \alpha}(-\alpha)=0
$$

and $\alpha \lambda-v>\lambda\left(\alpha-\min A_{u_{n}, \tau}\right) \geqslant 0$ for $\alpha \in A_{u_{n}, \tau}$ so

$$
\sum_{\alpha \in[1-\lambda+\nu, 1) \cap A_{u_{n}, \tau}} a_{u_{n}, \tau, \alpha}(1-\alpha)+\sum_{\alpha \in(\nu, 1-\lambda+\nu) \cap A_{u_{n}, \tau}} a_{u_{n}, \tau, \alpha} \frac{\alpha \lambda-v}{1-\lambda}>0
$$

hence, $v$ does not solve (4.21). Next, suppose that $v>\lambda \max A_{u_{n}, \tau}$. Then, we have $\max A_{u_{n}, \tau}=$ $(1-\lambda) \max A_{u_{n}, \tau}+\lambda \max A_{u_{n}, \tau}<1-\lambda+v$ so

$$
\sum_{\alpha \in[1-\lambda+v, 1) \cap A_{u_{n}, \tau}} a_{u_{n}, \tau, \alpha}(1-\alpha)=0
$$


and $\alpha \lambda-v<\lambda\left(\alpha-\max A_{u_{n}, \tau}\right) \leqslant 0$ for $\alpha \in A_{u_{n}, \tau}$ so

$$
\sum_{\alpha \in(v, 1-\lambda+v) \cap A_{u_{n}, \tau}} a_{u_{n}, \tau, \alpha} \frac{\alpha \lambda-v}{1-\lambda}+\sum_{\alpha \in(0, v] \cap A_{u_{n}, \tau}} a_{u_{n}, \tau, \alpha}(-\alpha)<0 .
$$

Thus, if $v$ solves (4.21), we must have $v \in\left[\lambda \min A_{u_{n}, \tau}, \lambda \max A_{u_{n}, \tau}\right]$.

Note If $M=0$, then $u^{*}=\mathbf{0}=u_{n}$, which is satisfied if and only if $v \geqslant\left(e^{-\tau \Delta} u_{n}\right)_{i}=0$. If $M=$ $\langle\mathbf{1}, \mathbf{1}\rangle_{\mathcal{V}}$, then $u^{*}=\mathbf{1}=u_{n}$, which is satisfied if and only if $v \leqslant\left(e^{-\tau \Delta} u_{n}\right)_{i}-1+\lambda=\lambda$. Hence, we can always assume $v$ to lie in $[0, \lambda]$.

\subsection{Behaviour as $\lambda \uparrow 1$}

Usefully, for $\lambda<1,(4.2)$ is strictly convex, so it has a unique solution $u_{n+1}^{\lambda}$. In this section, we show that as $\lambda \uparrow 1$, these solutions converge, yielding a choice function for solutions of (4.3). By the discussion in Section 4.3, we have the following theorem.

Theorem 4.19 For $0 \leqslant \lambda<1$, (4.2) has a unique solution

$$
\left(u_{n+1}^{\lambda}\right)_{i}= \begin{cases}0, & \text { if and only if } v \geqslant\left(e^{-\tau \Delta} u_{n}\right)_{i}, \\ \frac{\left(e^{-\tau \Delta} u_{n}\right)_{i}-v}{1-\lambda}, & \text { if and only if }\left(e^{-\tau \Delta} u_{n}\right)_{i}-(1-\lambda)<v<\left(e^{-\tau \Delta} u_{n}\right)_{i}, \\ 1, & \text { if and only if } v \leqslant\left(e^{-\tau \Delta} u_{n}\right)_{i}-(1-\lambda),\end{cases}
$$

where $v$ is a solution to (4.20) and hence $v \in[0, \lambda]$.

As a prelude to investigating the convergence properties of $u_{n+1}^{\lambda}$, we first show that convergence of solutions of (4.2) as $\lambda \uparrow 1$ is relevant to solving (4.3).

Theorem 4.20 Fix $u_{n}$ and denote the objective function in (4.2) by:

$$
q_{\lambda}: u \mapsto(1-\lambda)\|u\|_{\mathcal{V}}^{2}-2\left\langle u, e^{-\tau \Delta} u_{n}\right\rangle \mathcal{V}
$$

Then as $\lambda \uparrow 1, q_{\lambda} \rightarrow q_{1}$ uniformly on $X$, and note that $q_{1}$ is equivalent to the objective function in (4.3). Furthermore, if $\left(u^{\lambda}\right) \in X$ solve (4.2) and $u^{\lambda} \rightarrow u$ as $\lambda \uparrow 1$, then $u \in X$ is a solution to (4.3).

Proof For any $u \in X$ and $\lambda \leqslant 1$,

$$
\left|q_{\lambda}(u)-q_{1}(u)\right|=\left.(1-\lambda)|| u\right|_{\mathcal{V}} ^{2} \leqslant(1-\lambda)\|\mathbf{1}\|_{\mathcal{V}}^{2}
$$

which tends to zero uniformly as $\lambda \uparrow 1$.

Next, suppose $u^{\lambda} \rightarrow u$ as above. Then, $u \in X$ since $X$ is closed. By uniform convergence, for all $\varepsilon>0$, we have some $\delta>0$ such that for all $\lambda \in(1-\delta, 1)$ and all $v \in X$

$$
\left|q_{\lambda}(v)-q_{1}(v)\right| \leqslant \varepsilon / 2
$$

Therefore, since the $u^{\lambda}$ minimises $q_{\lambda}$, for any $v \in X$, we have

$$
q_{1}\left(u^{\lambda}\right)-\varepsilon / 2 \leqslant q_{\lambda}\left(u^{\lambda}\right) \leqslant q_{\lambda}(v) \leqslant q_{1}(v)+\varepsilon / 2 .
$$


Since $q_{1}$ is continuous, we can take $\lambda \uparrow 1$ and rearrange to get

$$
q_{1}(u) \leqslant q_{1}(v)+\varepsilon
$$

and since $\varepsilon$ was arbitrary, we must have that $u$ is a minimiser of $q_{1}$.

Theorem 4.21 Suppose $M=\mathcal{M}\left(u_{n}\right) \in(0, \mathcal{M}(\mathbf{1}))$, and take $k$ as in Theorem 4.16 with

$$
\sum_{l=k+1}^{K} a_{u_{n}, \tau, \alpha_{l}}<M \leqslant \sum_{l=k}^{K} a_{u_{n}, \tau, \alpha_{l}} .
$$

Then for some sufficiently small $\delta>0$, depending only on $e^{-\tau \Delta} u_{n}$, and each $\lambda \in(1-\delta, 1)$

$$
\left(u_{n+1}^{\lambda}\right)_{i}= \begin{cases}0, & \text { if and only if }\left(e^{-\tau \Delta} u_{n}\right)_{i} \leqslant \alpha_{k-1}, \\ a_{u_{n}, \tau, \alpha_{k}}^{-1}\left(M-\sum_{\ell=k+1}^{K} a_{u_{n}, \tau, \alpha_{\ell}}\right), & \text { if and only if }\left(e^{-\tau \Delta} u_{n}\right)_{i}=\alpha_{k}, \\ 1, & \text { if and only if }\left(e^{-\tau \Delta} u_{n}\right)_{i} \geqslant \alpha_{k+1},\end{cases}
$$

and thus $u_{n+1}^{\lambda}$ converges to the RHS of (4.24) as $\lambda \uparrow 1$.

Proof As $A_{u_{n}, \tau}$ is a finite set, we can take $\delta>0$ sufficiently small so that the $\delta$-balls around the $\alpha \in A_{u_{n}, \tau}$ are disjoint. Let $\lambda \in(1-\delta, 1)$ and choose $v$ solving (4.20). Then by Proposition 4.18, $v \in(0, \lambda)$, by $(4.20)$ we have

$$
M=\sum_{\alpha \in A_{u_{n}, \tau}} a_{u_{n}, \tau, \alpha} \begin{cases}1, & v \leqslant \alpha-(1-\lambda), \\ \frac{\alpha-\nu}{1-\lambda}, & \alpha-(1-\lambda)<v<\alpha, \\ 0, & v \geqslant \alpha,\end{cases}
$$

and by choice of $\delta, v$ is within $1-\lambda$ of at most one $\alpha$. Let $\alpha_{0}:=0$ and $\alpha_{K+1}:=1$. Then, there exists $1 \leqslant m \leqslant K$ such that $v \in\left(\alpha_{m-1}, \alpha_{m+1}-(1-\lambda)\right)$, since these intervals cover $(0, \lambda)$, and we have

$$
M=\sum_{\ell=m+1}^{K} a_{u_{n}, \tau, \alpha_{l}}+a_{u_{n}, \tau, \alpha_{m}} \max \left\{\min \left\{\frac{\alpha_{m}-v}{1-\lambda}, 1\right\}, 0\right\} .
$$

Hence, by (4.23), we must have either $m=k$ if $v<\alpha_{m}$ or $m=k-1$ if $v \geqslant \alpha_{m}$. If $v<\alpha_{m}$,

$$
\frac{\alpha_{k}-v}{1-\lambda}=a_{u_{n}, \tau, \alpha_{k}}^{-1}\left(M-\sum_{\ell=k+1}^{K} a_{u_{n}, \tau, \alpha_{\ell}}\right),
$$

which by (4.22) gives (4.24). If $v \in\left[\alpha_{m}, \alpha_{m+1}-(1-\lambda)\right)$, then by (4.22) and since $m=k-1$

$$
\left(u_{n+1}^{\lambda}\right)_{i}= \begin{cases}0, & \text { if and only if }\left(e^{-\tau \Delta} u_{n}\right)_{i} \leqslant \alpha_{m}=\alpha_{k-1}, \\ 1, & \text { if and only if }\left(e^{-\tau \Delta} u_{n}\right)_{i} \geqslant \alpha_{m+1}=\alpha_{k} .\end{cases}
$$

Therefore

$$
M=\sum_{\ell=k}^{K} a_{u_{n}, \tau, \alpha_{\ell}}
$$


so it follows that

$$
a_{u_{n}, \tau, \alpha_{k}}^{-1}\left(M-\sum_{\ell=k+1}^{K} a_{u_{n}, \tau, \alpha_{\ell}}\right)=1
$$

and so (4.24) follows.

Note If $M=0$ or $M=\mathcal{M}(\mathbf{1})$, then the $u_{n+1}^{\lambda}$ can only be $\mathbf{0}$ or only be $\mathbf{1}$, respectively, and the convergence is trivial, so the supposition on $M$ incurs no loss of generality.

Note The RHS of (4.24) can immediately be seen to solve (4.3) as it satisfies the conditions of (4.10). Furthermore, note that $u_{n+1}^{\lambda}$ converges to a point in $\operatorname{Ext} X$ (i.e. the RHS of (4.24) is in $\operatorname{Ext} X)$ if and only if (4.11) holds, i.e. if and only if (4.3) has a unique solution and $u_{n+1}^{\lambda}$ converges to the unique solution of (4.3).

\subsection{The converse of Theorem 4.3}

In this section, we prove the following theorem.

Theorem 4.22 If $u=u_{n+1}$ solves (4.2), then $\exists \beta \in \mathcal{B}(u)$ (given by (4.26) when $\lambda=1$ and (4.28) when $0 \leqslant \lambda<1)$, such that $(u, \beta)$ is a solution to $(4.1)\left(\right.$ for $\beta$ as $\left.\beta_{n+1}\right)$.

Note If $(u, \beta)$ and $\left(u, \beta^{\prime}\right)$ solve (4.1), then rearranging we get

$$
\beta-\beta^{\prime}=\bar{\beta} \mathbf{1}-\bar{\beta}^{\prime} \mathbf{1}
$$

i.e. $\beta$ and $\beta^{\prime}$ differ only by a multiple of 1 . So, for a given $u$ and $\beta \in \mathcal{B}(u),(u, \beta)$ is a solution if and only if $\left(u, \beta^{\prime}\right)$ is a solution for all and only the $\beta^{\prime} \in\{\beta+\theta \mathbf{1} \mid \theta \in \mathbb{R}\} \cap \mathcal{B}(u)$. If $u_{i} \in(0,1)$ for an $i \in V$ and $(u, \beta)$ and $\left(u, \beta^{\prime}\right)$ solve (4.1), then $\beta=\beta^{\prime}$ as $\beta_{i}=\beta_{i}^{\prime}=0$.

4.5.1 $\lambda=1$

If $M=0$, then $u=u_{n}=\mathbf{0}$, is trivially a solution to (4.1), for e.g. $\beta=\mathbf{0}$, hence WLOG we can suppose $M=\mathcal{M}\left(u_{n}\right)>0$. Let $k$ be as in Theorem 4.16, such that

$$
\sum_{\ell=k+1}^{K} a_{u_{n}, \tau, \alpha_{\ell}}<M \leqslant \sum_{\ell=k}^{K} a_{u_{n}, \tau, \alpha_{\ell}} .
$$

Then, recalling Theorem 4.16, any solution $u$ to (4.2) for $\lambda=1$ must satisfy

$$
\begin{aligned}
& u_{i}=0, \text { if }\left(e^{-\tau \Delta} u_{n}\right)_{i}<\alpha_{k}, \\
& u_{i}=1, \text { if }\left(e^{-\tau \Delta} u_{n}\right)_{i}>\alpha_{k}, \\
& M-\sum_{\ell=k+1}^{K} a_{u_{n}, \tau, \alpha_{\ell}}=\sum_{\left(e^{-\tau \Delta} u_{n}\right)_{i}=\alpha_{k}} d_{i}^{r} u_{i} .
\end{aligned}
$$

For $\lambda=1,(4.1)$ becomes

$$
-e^{-\tau \Delta} u_{n}+\frac{M}{\langle\mathbf{1}, \mathbf{1}\rangle_{\mathcal{V}}} \mathbf{1}=\beta-\frac{\langle\beta, \mathbf{1}\rangle_{\mathcal{V}}}{\langle\mathbf{1}, \mathbf{1}\rangle_{\mathcal{V}}} \mathbf{1}
$$


We seek to find a $\beta$ such that $\beta_{i}=0$ if $u_{i} \in(0,1)$. Note that if $u_{i} \in(0,1)$, then by Theorem 4.16 , we have $\left(e^{-\tau \Delta} u_{n}\right)_{i}=\alpha_{k}$, so we desire to have

$$
-\alpha_{k}+\frac{M}{\langle\mathbf{1}, \mathbf{1}\rangle_{\mathcal{V}}}=-\frac{\langle\beta, \mathbf{1}\rangle_{\mathcal{V}}}{\langle\mathbf{1}, \mathbf{1}\rangle_{\mathcal{V}}}
$$

Therefore substituting into (4.25), we have candidate solution:

$$
\beta=\alpha_{k} \mathbf{1}-e^{-\tau \Delta} u_{n}
$$

We now verify that this candidate solution works even for binary $u$.

Proof of Theorem 4.22 for $\lambda=1$. We check that the $\beta$ as in (4.26) solves (4.25):

$$
-e^{-\tau \Delta} u_{n}+\frac{M}{\langle\mathbf{1}, \mathbf{1}\rangle_{\mathcal{V}}} \mathbf{1}=\alpha_{k} \mathbf{1}-e^{-\tau \Delta} u_{n}-\alpha_{k} \mathbf{1}+\frac{\left\langle e^{-\tau \Delta} u_{n}, \mathbf{1}\right\rangle_{\mathcal{V}}}{\langle\mathbf{1}, \mathbf{1}\rangle_{\mathcal{V}}} \mathbf{1}
$$

Moreover, by the form for $u$ from Theorem 4.16, it follows that $\beta \in \mathcal{B}(u)$.

\section{$4.5 .20 \leqslant \lambda<1$}

For $0 \leqslant \lambda<1,(4.2)$ is strictly convex, so recalling (4.22), it has unique solution

$$
u_{i}= \begin{cases}0, & \text { if and only if } v \geqslant\left(e^{-\tau \Delta} u_{n}\right)_{i}, \\ \frac{\left(e^{-\tau \Delta} u_{n}\right)_{i}-v}{1-\lambda}, & \text { if and only if }\left(e^{-\tau \Delta} u_{n}\right)_{i}-(1-\lambda)<v<\left(e^{-\tau \Delta} u_{n}\right)_{i}, \\ 1, & \text { if and only if } v \leqslant\left(e^{-\tau \Delta} u_{n}\right)_{i}-(1-\lambda),\end{cases}
$$

where $v \in[0, \lambda]$ solving (4.20) is such that $\bar{u}=\overline{u_{n}}$. Hence, (4.1) is satisfied if and only if for all $i \in V$

$$
\lambda \beta_{i}-\lambda \bar{\beta}=\lambda \bar{u}+ \begin{cases}-\left(e^{-\tau \Delta} u_{n}\right)_{i}, & \text { if } v \geqslant\left(e^{-\tau \Delta} u_{n}\right)_{i}, \\ -v, & \text { if }\left(e^{-\tau \Delta} u_{n}\right)_{i}-(1-\lambda)<v<\left(e^{-\tau \Delta} u_{n}\right)_{i}, \\ 1-\lambda-\left(e^{-\tau \Delta} u_{n}\right)_{i}, & \text { if } v \leqslant\left(e^{-\tau \Delta} u_{n}\right)_{i}-(1-\lambda) .\end{cases}
$$

We seek a $\beta$ solving this with $\beta_{i}=0$ if $u_{i} \in(0,1)$. Suppose $\exists i \in V$ for which $u_{i} \in(0,1)$. This occurs when $\left(e^{-\tau \Delta} u_{n}\right)_{i}-(1-\lambda)<v<\left(e^{-\tau \Delta} u_{n}\right)_{i}$, and so at this $i$ :

$$
-\lambda \bar{\beta}=\lambda \bar{u}-v \text {. }
$$

Plugging into (4.27), we get the candidate solution:

$$
\beta_{i}=\lambda^{-1} \begin{cases}v-\left(e^{-\tau \Delta} u_{n}\right)_{i}, & \text { if } v \geqslant\left(e^{-\tau \Delta} u_{n}\right)_{i}, \\ 0, & \text { if }\left(e^{-\tau \Delta} u_{n}\right)_{i}-(1-\lambda)<v<\left(e^{-\tau \Delta} u_{n}\right)_{i}, \\ v-\left(e^{-\tau \Delta} u_{n}\right)_{i}+1-\lambda, & \text { if } v \leqslant\left(e^{-\tau \Delta} u_{n}\right)_{i}-(1-\lambda),\end{cases}
$$

which obeys $\beta \in \mathcal{B}(u)$ since $u$ obeys (4.22).

Proof of Theorem 4.22 for $0 \leqslant \lambda<1$. By the above discussion, taking $u$ as in (4.22) and $\beta$ as in (4.28) entails that $(u, \beta)$ is a solution to (4.1) if $\exists i \in V$ with $u_{i} \in(0,1)$. We check the alternative 
case, i.e. for all $i \in V, u_{i} \in\{0,1\}$. Take $\beta$ as in (4.28). As $u$ is binary, either $v \geqslant\left(e^{-\tau \Delta} u_{n}\right)_{i}$ or $v \leqslant\left(e^{-\tau \Delta} u_{n}\right)_{i}-(1-\lambda)$ at each $i \in V$, so

$$
\lambda \beta=v \mathbf{1}-e^{-\tau \Delta} u_{n}+(1-\lambda) \chi_{\left\{i \mid\left(e^{-\tau \Delta} u_{n}\right)_{i} \geqslant \nu+1-\lambda\right\}} .
$$

But as $u$ is binary, we have $u=\chi_{\left\{i \mid\left(e^{-\tau \Delta} u_{n}\right)_{i} \geqslant v+1-\lambda\right\}}$ and so

$$
\lambda \bar{\beta}=v-\bar{u}+(1-\lambda) \bar{u}=v-\lambda \bar{u} .
$$

Thus, $\beta$ solves (4.27). Therefore, $(u, \beta)$ is always a solution to (4.1).

\subsection{Sufficient conditions for pinning}

Similarly to the AC flow, the semi-discrete scheme experiences pinning (if $\tau$ is taken too small). Results giving sufficient conditions for 'too small' in the non-mass-conserving case are proved in [38, Theorem 4.2] (for the MBO scheme) and [14, Theorem 4.5] (for the semi-discrete scheme). We here prove similar results in the mass-conserving case.

Lemma 4.23 For any $S \subseteq V$ and $\alpha \geqslant 0$, if

$$
\tau<\|\Delta\|^{-1} \log \left(1+\alpha \sqrt{\frac{\min _{i \in V} d_{i}^{r}}{\mathcal{M}\left(\chi_{S}\right)}}\right) \text { or } \tau<\alpha\left\|\Delta \chi_{S}\right\|_{\infty}^{-1},
$$

then $\left\|e^{-\tau \Delta} \chi_{S}-\chi_{S}\right\|_{\infty}<\alpha$, and if

$$
\tau \leqslant\|\Delta\|^{-1} \log \left(1+\alpha \sqrt{\frac{\min _{i \in V} d_{i}^{r}}{\mathcal{M}\left(\chi_{S}\right)}}\right) \text { or } \tau \leqslant \alpha\left\|\Delta \chi_{S}\right\|_{\infty}^{-1},
$$

then $\left\|e^{-\tau \Delta} \chi_{S}-\chi_{S}\right\|_{\infty} \leqslant \alpha$.

Proof Follows as a corollary of the proof of [38, Theorem 4.2].

Theorem 4.24 If $S \subseteq V$ and $\tau$ obeys (4.29) for $\alpha=\frac{1}{2}$, then $S_{\tau, \chi_{S}}=\left\{\chi_{S}\right\}$.

Proof By Lemma 4.23, we have that $\left\|e^{-\tau \Delta} \chi_{S}-\chi_{S}\right\|_{\infty}<\frac{1}{2}$ and it follows that $\max _{i \in S^{c}}\left(e^{-\tau \Delta} \chi_{S}\right)_{i}<\frac{1}{2}<\min _{i \in S}\left(e^{-\tau \Delta} \chi_{S}\right)_{i}$. Recall from Corollary 4.12 that $S_{\tau, \chi_{S}} \cap \operatorname{Ext} X$ is nonempty, so consider an arbitrary $u \in S_{\tau, \chi_{S}} \cap \operatorname{Ext} X$. By Corollary 4.14, to prove the theorem it will suffice to prove that $u$ must equal $\chi_{S}$.

By Theorem 4.13, if $u_{i}>0$ and $u_{j}<1$, then $\left(e^{-\tau \Delta} \chi_{S}\right)_{i} \geqslant\left(e^{-\tau \Delta} \chi_{S}\right)_{j}$. Thus, if $i \in S^{c}$ and $j \in S$, then $\left(e^{-\tau \Delta} \chi_{S}\right)_{i}<\frac{1}{2}<\left(e^{-\tau \Delta} \chi_{S}\right)_{j}$ and hence $u_{i}=0$ or $u_{j}=1$. Suppose that $u_{j}<1$ for some $j \in S$, then by the above $u_{i}=0$ for all $i \in S^{c}$. But then $u \leqslant \chi_{S}$ vertexwise and $u_{j}<\left(\chi_{S}\right)_{j}$, so $\mathcal{M}(u)<$ $\mathcal{M}\left(\chi_{S}\right)$, a contradiction. Hence, $u_{j}=1$ for all $j \in S$. Likewise, $u_{i}=0$ for all $i \in S^{c}$.

Theorem 4.25 If $S \subseteq V, \lambda \in[0,1)$, $\tau$ obeys (4.30) for $\alpha=\frac{1}{2} \lambda$ and $u$ solves (4.2) with $u_{n}=\chi_{S}$, then $u=\chi_{S}$.

Proof Recall that solutions to (4.2) are unique for $\lambda \in[0,1)$, so it suffices to show that $u=\chi_{S}$ is a valid solution. By Lemma 4.23, we have that $\left\|e^{-\tau \Delta} \chi_{S}-\chi_{S}\right\|_{\infty} \leqslant \frac{1}{2} \lambda$, and hence $\left(e^{-\tau \Delta} \chi_{S}\right)_{i} \leqslant$ 
$\frac{1}{2} \lambda$ if $i \in S^{c}$ and $\left(e^{-\tau \Delta} \chi_{S}\right)_{i} \geqslant 1-\frac{1}{2} \lambda$ if $i \in S$. Hence, taking $v=\frac{1}{2} \lambda$, we observe that (recalling that $\left.M:=\mathcal{M}\left(u_{n}\right)=\mathcal{M}\left(\chi_{S}\right)\right)$

$$
\begin{aligned}
& M+\sum_{i} d_{i}^{r} \begin{cases}-1, & v \leqslant\left(e^{-\tau \Delta} u_{n}\right)_{i}-(1-\lambda), \\
\frac{v-\left(e^{-\tau \Delta} u_{n}\right)_{i}}{1-\lambda}, & \left(e^{-\tau \Delta} u_{n}\right)_{i}-(1-\lambda)<v<\left(e^{-\tau \Delta} u_{n}\right)_{i}, \\
0, & v \geqslant\left(e^{-\tau \Delta} u_{n}\right)_{i},\end{cases} \\
& =M+\sum_{i} d_{i}^{r} \begin{cases}-1, & 1-\frac{1}{2} \lambda \leqslant\left(e^{-\tau \Delta} u_{n}\right)_{i}, \\
\frac{\nu-\left(e^{-\tau \Delta} u_{n}\right)_{i}}{1-\lambda}, & \left(e^{-\tau \Delta} u_{n}\right)_{i} \in\left(\frac{1}{2} \lambda, 1-\frac{1}{2} \lambda\right), \\
0, & \frac{1}{2} \lambda \geqslant\left(e^{-\tau \Delta} u_{n}\right)_{i},\end{cases} \\
& =M+\sum_{i} d_{i}^{r} \begin{cases}-1, & i \in S, \\
0, & i \in S^{c},\end{cases} \\
& =M-\mathcal{M}\left(\chi_{S}\right)=0 .
\end{aligned}
$$

Thus, $v=\frac{1}{2} \lambda$ solves (4.19), and so by (4.18) $u$ is given by

$$
\begin{aligned}
u_{i} & = \begin{cases}0, & \text { if and only if } v \geqslant\left(e^{-\tau \Delta} u_{n}\right)_{i}, \\
\frac{\left(e^{-\tau \Delta} u_{n}\right)_{i}-v}{1-\lambda}, & \text { if and only if }\left(e^{-\tau \Delta} u_{n}\right)_{i}-(1-\lambda)<v<\left(e^{-\tau \Delta} u_{n}\right)_{i}, \\
1, & \text { if and only if } v \leqslant\left(e^{-\tau \Delta} u_{n}\right)_{i}-(1-\lambda),\end{cases} \\
& = \begin{cases}0, & \text { if and only if } \frac{1}{2} \lambda \geqslant\left(e^{-\tau \Delta} u_{n}\right)_{i}, \\
\frac{\left(e^{-\tau \Delta} u_{n}\right)_{i}-\frac{1}{2} \lambda}{1-\lambda}, & \text { if and only if }\left(e^{-\tau \Delta} u_{n}\right)_{i} \in\left(\frac{1}{2} \lambda, 1-\frac{1}{2} \lambda\right), \\
1, & \text { if and only if } 1-\frac{1}{2} \lambda \leqslant\left(e^{-\tau \Delta} u_{n}\right)_{i},\end{cases} \\
& = \begin{cases}0, & \text { if and only if } i \in S^{c}, \\
1, & \text { if and only if } i \in S,\end{cases}
\end{aligned}
$$

so $u=\chi_{S}$ is a valid solution.

Note The sufficient bounds on $\tau$ derived here are exactly the same bounds as the ones derived in [14] and [38] for the non-mass-conserving case.

\subsection{A Lyapunov functional for the mass-conserving semi-discrete scheme}

In this section, we show that the Lyapunov functional for the semi-discrete scheme derived in [14, Section 4.2] is also a Lyapunov functional for the mass-conserving semi-discrete scheme. We then use this functional to examine the eventual behaviour of the scheme, extending the analysis in [14] by accounting for the complications that arise due to the mass conservation condition. All results in this section assume only that the initial condition $u_{0} \in \mathcal{V}_{[0,1]}$ and are otherwise independent of the initial condition.

Recall from [38, Lemma 4.5] the Lyapunov functional for the ordinary MBO scheme, i.e. the strictly concave functional $J: \mathcal{V} \rightarrow \mathbb{R}$

$$
J(u):=\left\langle\mathbf{1}-u, e^{-\tau \Delta} u\right\rangle_{\mathcal{V}}
$$


with first variation at $u, L_{u}: \mathcal{V} \rightarrow \mathbb{R}$

$$
L_{u}(v):=\left\langle v, \mathbf{1}-2 e^{-\tau \Delta} u\right\rangle_{\mathcal{V}}
$$

Theorem 4.26 (Cf. [14, Theorem 4.9]) When $0 \leqslant \lambda \leqslant 1$, the functional (on $\left.\mathcal{V}_{[0,1]}\right)$

$$
H(u):=J(u)+(\lambda-1)\langle u, \mathbf{1}-u\rangle_{\mathcal{V}}=\lambda\langle u, \mathbf{1}-u\rangle_{\mathcal{V}}+\left\langle u,\left(I-e^{-\tau \Delta}\right) u\right\rangle_{\mathcal{V}}
$$

is non-negative, and furthermore the functional is a Lyapunov functional for (4.1), in the sense that $H\left(u_{n+1}\right) \leqslant H\left(u_{n}\right)$ with equality if and only if $u_{n+1}=u_{n}$ for the sequence of $u_{n} \in \mathcal{V}_{[0,1]}$ defined by (4.1). Moreover, we have that

$$
H\left(u_{n}\right)-H\left(u_{n+1}\right) \geqslant(1-\lambda)\left\|u_{n+1}-u_{n}\right\|_{\mathcal{V}}^{2} .
$$

Proof Note that $I-e^{-\tau \Delta}$ has eigenvalues $1-e^{-\tau \gamma_{k}} \geqslant 0$, since the eigenvalues $\gamma_{k}$ of $\Delta$ are non-negative, and so $\left\langle u,\left(I-e^{-\tau \Delta}\right) u\right\rangle_{\mathcal{V}} \geqslant 0$. As $u \in \mathcal{V}_{[0,1]}, H(u) \geqslant 0$ follows.

Next by the concavity of $J$ and linearity of $L_{u_{n}}$, recalling that $\left\langle u_{n}-u_{n+1}, \mathbf{1}\right\rangle_{\mathcal{V}}=0$ :

$$
\begin{aligned}
H\left(u_{n}\right)- & H\left(u_{n+1}\right)=J\left(u_{n}\right)-J\left(u_{n+1}\right)+(1-\lambda)\left\langle u_{n+1}, \mathbf{1}-u_{n+1}\right\rangle_{\mathcal{V}}-(1-\lambda)\left\langle u_{n}, \mathbf{1}-u_{n}\right\rangle_{\mathcal{V}} \\
& \geqslant L_{u_{n}}\left(u_{n}-u_{n+1}\right)-(1-\lambda)\left\langle u_{n+1}, u_{n+1}\right\rangle_{\mathcal{V}}+(1-\lambda)\left\langle u_{n}, u_{n}\right\rangle_{\mathcal{V}} \\
& =\left\langle u_{n}-u_{n+1}, \mathbf{1}-2 e^{-\tau \Delta} u_{n}\right\rangle_{\mathcal{V}}-(1-\lambda)\left\langle u_{n+1}, u_{n+1}\right\rangle_{\mathcal{V}}+(1-\lambda)\left\langle u_{n}, u_{n}\right\rangle_{\mathcal{V}} \\
& =\left\langle u_{n}-u_{n+1},-2 e^{-\tau \Delta} u_{n}+(1-\lambda)\left(u_{n+1}+u_{n}\right)\right\rangle_{\mathcal{V}} \\
& =\left\langle u_{n}-u_{n+1}, 2(1-\lambda) u_{n+1}-2 e^{-\tau \Delta} u_{n}+(1-\lambda)\left(u_{n}-u_{n+1}\right)\right\rangle_{\mathcal{V}} \\
& =\left\langle u_{n}-u_{n+1}, 2 \lambda \beta_{n+1}-2 \lambda \overline{u_{n+1}} \mathbf{1}-2 \lambda \overline{\beta_{n+1}} \mathbf{1}+(1-\lambda)\left(u_{n}-u_{n+1}\right)\right\rangle_{\mathcal{V}} \text { by (4.1) } \\
& =\left\langle u_{n}-u_{n+1}, 2 \lambda \beta_{n+1}\right\rangle_{\mathcal{V}}+(1-\lambda)\left\|u_{n+1}-u_{n}\right\|_{\mathcal{V}}^{2} \\
& \geqslant(1-\lambda)\left\|u_{n+1}-u_{n}\right\|_{\mathcal{V}}^{2}
\end{aligned}
$$

where the final line follows from $\beta_{n+1} \in \mathcal{B}\left(u_{n+1}\right)$ as in the proof of Theorem 4.3. Note that if $u_{n+1} \neq u_{n}$, then $J\left(u_{n}\right)-J\left(u_{n+1}\right)>L_{u_{n}}\left(u_{n}-u_{n+1}\right)$ by strict concavity, so even for $\lambda=1$, there is equality if and only if $u_{n+1}=u_{n}$.

Corollary 4.27 (Cf. [37, Lemma 5.18]) Recall from Definition 4.9 the notation $S_{\tau, u_{n}}$ for the set of valid MBO updates of $u_{n}$, i.e. the set of solutions to (4.3). For $\lambda=1$, if an MBO sequence $u_{n}$ defined by (4.3) satisfies either:

(i) for eventually all $n, u_{n+1} \in \operatorname{Ext} S_{\tau, u_{n}}$, or

(ii) for eventually all $n, u_{n+1}$ is as in (4.24) (i.e. the $\lambda \uparrow 1$ limit of the semi-discrete updates $\left.u_{n+1}^{\lambda}\right)$,

then there exists $u \in X$ such that for eventually all $n, u_{n}=u$.

Proof For (i), recall that Ext $S_{\tau, u_{n}}=S_{\tau, u_{n}} \cap \operatorname{Ext} X \subseteq \operatorname{Ext} X$ and that $\operatorname{Ext} X$ is a finite set. Hence, $\left\{u_{n} \mid n \in \mathbb{N}\right\}$ is a finite set, so if the $u_{n}$ are not eventually a single $u$, then we must have some 
$u, v \in X$ such that $u \neq v, u_{n}=u$ infinitely often, and $u_{n}=v$ infinitely often. Therefore, we must have $n<m<k$ such that $u_{n}=u_{k}=u$ and $u_{m}=v$, and hence

$$
H(u) \geqslant H\left(u_{n+1}\right) \geqslant \ldots \geqslant H\left(u_{m-1}\right) \geqslant H(v) \geqslant H\left(u_{m+1}\right) \geqslant \ldots \geqslant H\left(u_{k-1}\right) \geqslant H(u) .
$$

All the inequalities are equalities, and therefore by the equality condition on $H$ from Theorem 4.26 , we have $u=v$, a contradiction. Thus, the $u_{n}$ are eventually constant.

For (ii), we show that there are finitely many possible $u \in X$ of the form (4.24). Each such $u$ has the form

$$
u=\frac{M-\mathcal{M}\left(\chi_{V_{3}}\right)}{\mathcal{M}\left(\chi_{V_{2}}\right)} \chi_{V_{2}}+\chi_{V_{3}}
$$

for a partition $V=V_{1} \cup V_{2} \cup V_{3}$ with $0 \leqslant M-\mathcal{M}\left(\chi_{V_{3}}\right) \leqslant \mathcal{M}\left(\chi_{V_{2}}\right)$. To see this, note that $u$ as in (4.24) has $V_{1}=\left\{i \mid\left(e^{-\tau \Delta} u_{n}\right)_{i}<\alpha_{k}\right\}, V_{2}=\left\{i \mid\left(e^{-\tau \Delta} u_{n}\right)_{i}=\alpha_{k}\right\}$, and $V_{3}=\left\{i \mid\left(e^{-\tau \Delta} u_{n}\right)_{i}>\alpha_{k}\right\}$. But since $V$ is finite, there are only finitely many tripartitions of $V$. Hence, $\left\{u_{n} \mid n \in \mathbb{N}\right\}$ is a finite set, and the proof runs as above.

Corollary 4.28 (Cf. [14, Corollary 4.10]) If $\lambda \in(0,1)$ and the sequence $u_{n}$ obeys (4.1), then

$$
\sum_{n=0}^{\infty}\left\|u_{n+1}-u_{n}\right\|_{\mathcal{V}}^{2}<\infty
$$

and therefore in particular

$$
\lim _{n \rightarrow \infty}\left\|u_{n+1}-u_{n}\right\|_{\mathcal{V}}=0
$$

Proof By the non-negativity of $H$ and (4.32), we have

$$
(1-\lambda) \sum_{n=0}^{N}\left\|u_{n+1}-u_{n}\right\|_{\mathcal{V}}^{2} \leqslant H\left(u_{0}\right)-H\left(u_{N+1}\right) \leqslant H\left(u_{0}\right)
$$

so the result follows by taking $N \rightarrow \infty$.

We wish to use the gradient of $H$ to investigate critical points of the flow. However as we restrict the flow to lie in $S_{M}$, a non-Hilbert space, we make the following definition.

Definition 4.29 Let $H_{0}$ be a Hilbert space, and $H_{1} \subseteq H_{0}$ be a closed subspace. Let $\tilde{H}:=x+H_{1}$ for some $x \in H_{0}$. Then for any Fréchet differentiable map $f: H_{0} \rightarrow \mathbb{R}$ with Fréchet derivative $D f$, we define the Fréchet derivative of $\left.f\right|_{\tilde{H}}$ at $u \in \tilde{H}$ by

$$
\left.D f\right|_{\tilde{H}}(u):=\left.D f(u)\right|_{H_{1}},
$$

where the restriction of the argument to $H_{1}$ ensures that the $u+h$ terms that appear in the definition of the Fréchet derivative satisfy $u+h \in \tilde{H}$. Then, we define the gradient

$$
\left.\nabla_{\tilde{H}} f\right|_{\tilde{H}}(u) \in H_{1}
$$

to be the Riesz representative of $\left.D f\right|_{\tilde{H}}(u)$, i.e. the unique element of $H_{1}$ such that

$$
\forall v \in H_{1}\left\langle\left.\nabla_{\tilde{H}} f\right|_{\tilde{H}}(u), v\right\rangle=\left.D f\right|_{\tilde{H}}(u)(v)=D f(u)(v) .
$$


Note, therefore, that for $u \in \tilde{H}$, since $\nabla_{H_{0}} f(u)$ is the Riesz representative of $D f(u)$,

$$
\forall v \in H_{1}\left\langle\nabla_{H_{0}} f(u), v\right\rangle=\left\langle\left.\nabla_{\tilde{H}} f\right|_{\tilde{H}}(u), v\right\rangle
$$

and so $\left.\nabla_{\tilde{H}} f\right|_{\tilde{H}}(u)-\nabla_{H_{0}} f(u) \perp H_{1}$. That is, for $u \in \tilde{H},\left.\nabla_{\tilde{H}} f\right|_{\tilde{H}}(u)$ is the orthogonal projection of $\nabla_{H_{0}} f(u)$ onto $H_{1}$.

Proposition 4.30 (Cf. [14, Proposition 4.11]) Suppose $M \in(0, \mathcal{M}(\mathbf{1}))$. The Lyapunov functional has gradient (for $\left.u \in \mathcal{V}_{(0,1)} \cap X\right)$

$$
\left.\nabla_{S_{M}} H\right|_{S_{M}}(u)=2\left(u-e^{-\tau \Delta} u\right)-2 \lambda u+2 \lambda \bar{u} \mathbf{1}
$$

and therefore:

(i) For $u_{n+1} \in \mathcal{V}_{(0,1)} \cap X$ obeying (4.1)

$$
\left.\nabla_{S_{M}} H\right|_{S_{M}}\left(u_{n+1}\right)=-2 e^{-\tau \Delta}\left(u_{n+1}-u_{n}\right)
$$

(ii) Define $\mathscr{E}$ to be the eigenspace of $\Delta$ with eigenvalue $-\tau^{-1} \log (1-\lambda)$, or $\{\mathbf{0}\}$ if there is no such eigenvalue. If $u \in \mathcal{V}_{(0,1)} \cap X$, then $\left.\nabla_{S_{M}} H\right|_{S_{M}}(u)=\mathbf{0}$ (i.e. $u$ is a critical point of $H$ ) if and only if $u \in\left(\frac{M}{\langle\mathbf{1}, \mathbf{1}\rangle_{\mathcal{V}}} \mathbf{1}+\mathscr{E}\right) \cap \mathcal{V}_{(0,1)}$.

Proof It is straightforward to check that

$$
\left\langle\nabla_{\mathcal{V}} H(u), v\right\rangle_{\mathcal{V}}:=\lim _{t \rightarrow 0} \frac{H(u+t v)-H(u)}{t}=\left\langle 1-2 e^{-\tau \Delta} u, v\right\rangle_{\mathcal{V}}+(\lambda-1)\langle 1-2 u, v\rangle_{\mathcal{V}}
$$

and therefore

$$
\nabla_{\mathcal{V}} H(u)=1-2 e^{-\tau \Delta} u+(\lambda-1)(1-2 u)=\lambda-2 e^{-\tau \Delta} u+2(1-\lambda) u .
$$

Restricting to $S_{M}=u+\{\mathbf{1}\}^{\perp}$, by definition $\left.\nabla_{S_{M}} H\right|_{S_{M}}(u) \in\{\mathbf{1}\}^{\perp}$ and $\left.\nabla_{S_{M}} H\right|_{S_{M}}(u)-\nabla_{\mathcal{V}} H(u) \in$ $\operatorname{span}\{\mathbf{1}\}$. Thus, $\left.\nabla_{S_{M}} H\right|_{S_{M}}(u)=\nabla_{\mathcal{V}} H(u)-\overline{\nabla_{\mathcal{V}} H(u)} \mathbf{1}$, yielding (4.33).

(i) Since $u_{n+1} \in \mathcal{V}_{(0,1)}$, we have $\beta_{n+1}=\mathbf{0}$, so from (4.1), we have

$$
u_{n+1}-e^{-\tau \Delta} u_{n}-\lambda u_{n+1}+\lambda \overline{u_{n+1}} \mathbf{1}=\lambda \beta_{n+1}-\lambda \overline{\beta_{n+1}} \mathbf{1}=\mathbf{0}
$$

and (4.34) follows by substituting $u_{n+1}$ into (4.33) and subtracting twice the above expression.

(ii) Let $A: v \mapsto \bar{v} \mathbf{1}$ and define $B:=2 e^{-\tau \Delta}+2(\lambda-1) I-2 \lambda A$. Then, $\left.\nabla_{S_{M}} H\right|_{S_{M}}(u)=\mathbf{0}$ if and only if $B u=\mathbf{0}$. Note that $B \mathbf{1}=2 \mathbf{1}+2 \lambda \mathbf{1}-2 \mathbf{1}-2 \lambda \mathbf{1}=\mathbf{0}$ so $u=\frac{M}{\langle\mathbf{1 , 1}\rangle_{\mathcal{V}}} \mathbf{1} \in X$ is a solution. Taking $\left(\xi_{k}\right)_{k>0}$ the eigenvectors for $\Delta$ (with eigenvalues $\gamma_{k}$ ) as a basis for $\{\mathbf{1}\}^{\perp}$, we get that (recalling that $\xi_{k} \perp \mathbf{1}$ for $k>0$ and $\gamma_{k}>0$ for $k>0$ )

$$
B \xi_{k}=2\left(e^{-\tau \gamma_{k}}+\lambda-1\right) \xi_{k}=\mathbf{0} \text { if and only if } \xi_{k} \in \mathscr{E} .
$$

Thus, $B u=\mathbf{0}$ if and only if

$$
B\left(u-\frac{M}{\langle\mathbf{1}, \mathbf{1}\rangle_{\mathcal{V}}} \mathbf{1}\right)=\mathbf{0}
$$


if and only if

$$
u-\frac{M}{\langle\mathbf{1}, \mathbf{1}\rangle_{\mathcal{V}}} \mathbf{1} \in \mathscr{E}
$$

as desired.

Note We identify when the above identified critical points are all global maximisers. Considering the quadratic terms, we observe that for $\eta \perp \mathbf{1}$ (recalling $\bar{u}=M /\langle\mathbf{1}, \mathbf{1}\rangle_{\mathcal{V}}$ )

$$
\begin{aligned}
H(\bar{u} \mathbf{1}+\eta) & =\lambda\langle\bar{u} \mathbf{1}+\eta, \mathbf{1}-\bar{u} \mathbf{1}-\eta\rangle_{\mathcal{V}}+\left\langle\bar{u} \mathbf{1}+\eta,\left(I-e^{-\tau \Delta}\right)(\bar{u} \mathbf{1}+\eta)\right\rangle_{\mathcal{V}} \\
& =H(\bar{u} \mathbf{1})-\left(\left\langle\eta, e^{-\tau \Delta} \eta\right\rangle_{\mathcal{V}}-(1-\lambda)\langle\eta, \eta\rangle_{\mathcal{V}}\right)
\end{aligned}
$$

so, for $\lambda \leqslant 1, u=\frac{M}{\langle\mathbf{1 , 1}\rangle_{\mathcal{V}}} \mathbf{1}$ is a global maximiser of $H$ in $S_{M}$ if and only if

$$
P:=e^{-\tau \Delta}-(1-\lambda) I
$$

is positive semi-definite on $S_{M}$, i.e. for $\left(\gamma_{k}\right)_{k>0}$ the eigenvalues of $\Delta$, we desire that:

$$
e^{-\tau \gamma_{k}}-(1-\lambda) \geqslant 0 \text {, i.e. } \tau \varepsilon^{-1} \geqslant 1-e^{-\tau \gamma_{k}} \text {. }
$$

Therefore, we have for $\lambda \leqslant 1$ that $u=\frac{M}{\langle\mathbf{1}, \mathbf{1}\rangle \mathcal{V}} \mathbf{1}$ is a global maximiser of $H$ if and only if

$$
\varepsilon \in\left[\tau, \frac{\tau}{1-e^{-\tau\|\Delta\|}}\right] .
$$

Furthermore, note that $\mathscr{E}=\operatorname{ker} P$ so in that case $\frac{M}{\langle\mathbf{1}, \mathbf{1}\rangle_{\mathcal{V}}} \mathbf{1}+\mathscr{E}$ are all global maxima since $H\left(\frac{M}{\langle\mathbf{1}, \mathbf{1}\rangle \mathcal{V}} \mathbf{1}+\eta\right)=H\left(\frac{M}{\langle\mathbf{1}, \mathbf{1}\rangle \mathcal{V}} \mathbf{1}\right)$ for $\eta \in \operatorname{ker} P$.

Since $H\left(u_{n}\right)$ is monotonically decreasing and bounded below, it follows that $H\left(u_{n}\right) \downarrow H_{\infty}$ for some $H_{\infty} \geqslant 0$. Furthermore, since the sequence $u_{n}$ is contained in $X$ and $X$ is compact, there exists a subsequence $u_{n_{k}}$ that converges to some $u^{*} \in X$ with $H\left(u^{*}\right)=H_{\infty}$, since $H$ is continuous. Unfortunately, just like [29] for graph AC flow with the standard quartic potential, or [14] for AC flow with the double-obstacle potential, we are unable to infer convergence of the whole sequence from these facts. However, by the same argument as in [29, Lemma 5], if the set of accumulation points of the $u_{n}$ is finite, then there is in fact only one such point and the whole sequence converges. Notably, if $u^{*} \in \mathcal{V}_{(0,1)} \cap X$ is an accumulation point of the $u_{n}$ then by Corollary 4.28 and (4.34), we have that $\left.\nabla_{S_{M}} H\right|_{S_{M}}\left(u^{*}\right)=\mathbf{0}$. Thus, if $H\left(u_{0}\right)<H\left(\frac{M}{\langle\mathbf{1 , 1}\rangle_{\mathcal{V}}} \mathbf{1}\right)$, then no accumulation points of the $u_{n}$ lie in $\mathcal{V}_{(0,1)} \cap X$.

\section{Convergence of the semi-discrete scheme}

We follow the method of [14] to prove convergence of the semi-discrete iterates to the solution of the continuous-time flow (3.7) for $T=[0, \infty)$ and $u(0)=u_{0} \in \mathcal{V}_{[0,1]}$. Note that for $\overline{u_{0}} \in\{0,1\}$, this result is trivial, since the semi-discrete scheme has $u_{n} \equiv u_{0}$ and (3.7) has $u(t) \equiv u_{0}$. Therefore, for the rest of this section, we shall assume $\bar{u}=\overline{u_{0}} \in(0,1)$.

\subsection{Asymptotics of the $n$th semi-discrete iterate}

We first note two important controls. 
Lemma 5.1 For $0<\lambda \leqslant 1$, and $\left(u_{n+1}, \beta_{n+1}\right)$ solving (4.1) for given $u_{n} \in \mathcal{V}_{[0,1]}$, suppose $\bar{u}:=$ $\overline{u_{n}}=\overline{u_{n+1}} \in(0,1)$. Then

$$
\beta_{n+1}-\overline{\beta_{n+1}} \mathbf{1} \in \mathcal{V}_{[\bar{u}-1, \bar{u}]}
$$

and

$$
\beta_{n+1} \in \mathcal{V}_{[-1,1]}
$$

Proof First, suppose $\lambda=1$. Then by (4.25),

$$
\left(\beta_{n+1}\right)_{i}-\overline{\beta_{n+1}}=\bar{u}-\left(e^{-\tau \Delta} u_{n}\right)_{i} \in[\bar{u}-1, \bar{u}] .
$$

Next, suppose $\lambda \in(0,1)$. Then by (4.27),

$$
\begin{aligned}
\left(\beta_{n+1}\right)_{i}-\overline{\beta_{n+1}}=\bar{u}+\frac{1}{\lambda} \begin{cases}-\left(e^{-\tau \Delta} u_{n}\right)_{i}, & \text { if } v \geqslant\left(e^{-\tau \Delta} u_{n}\right)_{i}, \\
-v, & \text { if }\left(e^{-\tau \Delta} u_{n}\right)_{i}-(1-\lambda)<v<\left(e^{-\tau \Delta} u_{n}\right)_{i}, \\
1-\lambda-\left(e^{-\tau \Delta} u_{n}\right)_{i}, & \text { if } v \leqslant\left(e^{-\tau \Delta} u_{n}\right)_{i}-(1-\lambda),\end{cases} \\
=\bar{u}-1+\frac{1}{\lambda} \begin{cases}\lambda-\left(e^{-\tau \Delta} u_{n}\right)_{i}, & \text { if } v \geqslant\left(e^{-\tau \Delta} u_{n}\right)_{i}, \\
\lambda-v, & \text { if }\left(e^{-\tau \Delta} u_{n}\right)_{i}-(1-\lambda)<v<\left(e^{-\tau \Delta} u_{n}\right)_{i}, \\
1-\left(e^{-\tau \Delta} u_{n}\right)_{i}, & \text { if } v \leqslant\left(e^{-\tau \Delta} u_{n}\right)_{i}-(1-\lambda),\end{cases}
\end{aligned}
$$

where we recall from Proposition 4.18 that $v \in(0, \lambda)$. It is therefore easy to check that in the first line, the conditional term is non-positive, and in the second line, the conditional term is non-negative. Therefore, we deduce (5.1).

Consider the set $\mathscr{B}:=\left\{\left(\beta_{n+1}\right)_{i} \mid i \in V\right\}$. By $(5.1), \mathscr{B}-\overline{\beta_{n+1}} \subseteq[\bar{u}-1, \bar{u}]$, so we have that $\operatorname{diam} \mathscr{B} \leqslant 1$. Furthermore, $u_{n+1} \notin\{\mathbf{0}, \mathbf{1}\}$, so since $\beta_{n+1} \in \mathcal{B}\left(u_{n+1}\right)$, we have $x, y \in \mathscr{B}$ such that $x \geqslant 0$ and $y \leqslant 0$. Therefore, $\mathscr{B} \subseteq[x-1, x+1] \cap[y-1, y+1] \subseteq[-1,1]$.

Recall that the semi-discrete scheme is defined for $\lambda<1$ by

$$
(1-\lambda) u_{n+1}=e^{-\tau \Delta} u_{n}-\lambda \bar{u} \mathbf{1}+\lambda \beta_{n+1}-\lambda \overline{\beta_{n+1}} \mathbf{1} .
$$

Iterating this formula, we get the following formula for the $n$th term. For bookkeeping, we introduce the superscript $[\tau]$ to keep track of the time step governing a particular sequence of $u_{n}$ and $\beta_{n}$ - note that these can both be considered as functions of $(\tau, n)$.

Proposition 5.2 (Cf. [14, Proposition 5.1]) For $0<\lambda<1$, the semi-discrete solution has $n$th iterate

$$
u_{n}^{[\tau]}=\bar{u} \mathbf{1}+(1-\lambda)^{-n} e^{-n \tau \Delta}\left(u_{0}-\bar{u} \mathbf{1}\right)+\frac{\lambda}{1-\lambda} \sum_{k=1}^{n}(1-\lambda)^{-(n-k)} e^{-(n-k) \tau \Delta}\left(\beta_{k}^{[\tau]}-\overline{\beta_{k}^{[\tau]}} \mathbf{1}\right) .
$$

Therefore, understanding $\mathcal{O}$ to refer to the simultaneous limit of $\tau \downarrow 0$ and $n \rightarrow \infty$ with $n \tau-t \in[0, \tau)$ for some fixed $t \geqslant 0$ and for fixed $\varepsilon>0$ (i.e., $f(\tau, n)=\mathcal{O}(\tau)$ if and only if $\limsup |f(\tau, n) / \tau|<\infty$ as $(\tau, n) \rightarrow(0, \infty)$ in $\{(\rho, m) \mid \rho>0, m \rho-t \in[0, \rho)\}$ with the subspace 
topology induced by the standard topology on $(0, \infty) \times \mathbb{N})$, we have that with respect to this limit (recalling that $\lambda:=\tau / \varepsilon$ and hence $\lambda=\mathcal{O}(\tau)$ in this regime)

$$
u_{n}^{[\tau]}=\bar{u} \mathbf{1}+e^{n \lambda} e^{-n \tau \Delta}\left(u_{0}-\bar{u} \mathbf{1}\right)+\lambda \sum_{k=1}^{n} e^{(n-k) \lambda} e^{-(n-k) \tau \Delta}\left(\beta_{k}^{[\tau]}-\overline{\beta_{k}^{[\tau]}} \mathbf{1}\right)+\mathcal{O}(\tau) .
$$

Proof We follow the proof of [14, Proposition 5.1]. We prove the first claim by induction. The $n=0$ base case is trivial. Then writing $\theta_{n}^{[\tau]}:=\beta_{n}^{[\tau]}-{\overline{\beta_{n}^{[\tau]}}}_{1} \in \mathcal{V}_{[\bar{u}-1, \bar{u}]}$ (by Lemma 5.1) and inducting, we have:

$$
\begin{aligned}
u_{n+1}^{[\tau]}= & (1-\lambda)^{-1} e^{-\tau \Delta} u_{n}-\frac{\lambda}{1-\lambda} \bar{u} \mathbf{1}+\frac{\lambda}{1-\lambda} \theta_{n+1}^{[\tau]} \\
= & (1-\lambda)^{-1} e^{-\tau \Delta}\left[\bar{u} \mathbf{1}+(1-\lambda)^{-n} e^{-n \tau \Delta}\left(u_{0}-\bar{u} \mathbf{1}\right)\right. \\
& \left.+\frac{\lambda}{1-\lambda} \sum_{k=1}^{n}(1-\lambda)^{-(n-k)} e^{-(n-k) \tau \Delta} \theta_{k}^{[\tau]}\right]-\frac{\lambda}{1-\lambda} \bar{u} \mathbf{1}+\frac{\lambda}{1-\lambda} \theta_{n+1}^{[\tau]} \\
= & \left(\frac{1}{1-\lambda}-\frac{\lambda}{1-\lambda}\right) \bar{u} \mathbf{1}+(1-\lambda)^{-(n+1)} e^{-(n+1) \tau \Delta}\left(u_{0}-\bar{u} \mathbf{1}\right) \\
& +\frac{\lambda}{1-\lambda} \sum_{k=1}^{n}(1-\lambda)^{-(n-k+1)} e^{-(n-k+1) \tau \Delta} \theta_{k}^{[\tau]}+\frac{\lambda}{1-\lambda} \theta_{n+1}^{[\tau]} \\
= & \bar{u} \mathbf{1}+(1-\lambda)^{-(n+1)} e^{-(n+1) \tau \Delta}\left(u_{0}-\bar{u} \mathbf{1}\right)+\frac{\lambda}{1-\lambda} \sum_{k=1}^{n+1}(1-\lambda)^{-(n-k+1)} e^{-(n-k+1) \tau \Delta} \theta_{k}^{[\tau]}
\end{aligned}
$$

completing the induction.

To prove the second claim, we use that $n \tau=t+\mathcal{O}(\tau)$ and $n \lambda=t / \varepsilon+\mathcal{O}(\tau)$ for fixed $t$ to control:

$$
\begin{aligned}
Q & :=\left\|u_{n}^{[\tau]}-\bar{u} \mathbf{1}-e^{n \lambda} e^{-n \tau \Delta}\left(u_{0}-\bar{u} \mathbf{1}\right)-\lambda \sum_{k=1}^{n} e^{(n-k) \lambda} e^{-(n-k) \tau \Delta} \theta_{k}^{[\tau]}\right\|_{\mathcal{V}} \\
& =\left\|\left((1-\lambda)^{-n}-e^{n \lambda}\right)\left(e^{-n \tau \Delta} u_{0}-\bar{u} \mathbf{1}\right)+\lambda \sum_{k=1}^{n}\left((1-\lambda)^{-(n-k+1)}-e^{(n-k) \lambda}\right) e^{-(n-k) \tau \Delta} \theta_{k}^{[\tau]}\right\|_{\mathcal{V}} \\
& \leqslant\left((1-\lambda)^{-n}-e^{n \lambda}\right)\left\|e^{-n \tau \Delta} u_{0}-\bar{u} \mathbf{1}\right\|_{\mathcal{V}}+\lambda \sum_{k=1}^{n}\left((1-\lambda)^{-(n-k+1)}-e^{(n-k) \lambda}\right)\left\|e^{-(n-k) \tau \Delta} \theta_{k}^{[\tau]}\right\|_{\mathcal{V}} \\
& \leqslant\left((1-\lambda)^{-n}-e^{n \lambda}\right)\left(\left\|e^{-t \Delta} u_{0}-\bar{u} \mathbf{1}\right\|_{\mathcal{V}}+\mathcal{O}(\tau)\right)+\lambda C \sum_{k=1}^{n}\left((1-\lambda)^{-(n-k+1)}-e^{(n-k) \lambda}\right),
\end{aligned}
$$

with the first equality by the triangle inequality since $(1-\lambda)^{-(r+1)}-e^{r \lambda} \geqslant 0$ as $e^{-\lambda r /(r+1)} \geqslant 1-\lambda r /(r+1) \geqslant 1-\lambda$, and for the second inequality, we can take 
$C:=\sup _{s \in[0, t+\tau]} \sup _{\theta \in \mathcal{V}_{[\bar{u}-1, \bar{u}]}}\left\|e^{-s \Delta} \theta\right\|_{\mathcal{V}}$. Note that we can bound $C \leqslant \sup _{s \in[0, t+\tau]}\left\|e^{-s \Delta}\right\|$. $\max \{\bar{u}, 1-\bar{u}\}\|\mathbf{1}\|_{\mathcal{V}}=\max \{\bar{u}, 1-\bar{u}\}\|\mathbf{1}\|_{\mathcal{V}}$. Then

$R H S=(D+\mathcal{O}(\tau))\left((1-\lambda)^{-n}-e^{t / \varepsilon}\right)+C \frac{\lambda}{1-\lambda} \frac{(1-\lambda)^{-n}-1}{(1-\lambda)^{-1}-1}-\lambda C \frac{e^{n \lambda}-1}{e^{\lambda}-1}$,

where $D:=\left\|e^{-t \Delta} u_{0}-\bar{u} \mathbf{1} \mid\right\|_{\mathcal{V}}$, so noting that $(1-\lambda)^{-n}=(1-(t / \varepsilon+\mathcal{O}(\tau)) / n)^{-n}=e^{t / \varepsilon+\mathcal{O}(\tau)}+$ $\mathcal{O}(1 / n)=e^{t / \varepsilon}+\mathcal{O}(\tau)$,

$$
\begin{aligned}
R H S & =\mathcal{O}(\tau)+C\left(e^{t / \varepsilon}-1+\mathcal{O}(\tau)-\lambda \frac{e^{t / \varepsilon}-1}{e^{\lambda}-1}\right) \\
& =\mathcal{O}(\tau)+C\left(e^{t / \varepsilon}-1\right) \frac{e^{\lambda}-1-\lambda}{e^{\lambda}-1} \\
& =\mathcal{O}(\tau)+C\left(e^{t / \varepsilon}-1\right) \mathcal{O}(\lambda)=\mathcal{O}(\tau)
\end{aligned}
$$

as desired. We have used here that $\frac{e^{\lambda}-1-\lambda}{e^{\lambda}-1}$ has Taylor series $\frac{1}{2} \lambda-\frac{1}{12} \lambda^{2}+\mathcal{O}\left(\lambda^{4}\right)$, as can be checked by direct calculation.

\subsection{Proof of convergence}

We consider the limit of (5.4) as $\tau \downarrow 0, n \rightarrow \infty$ with $n \tau \rightarrow t$ for some fixed $t$ and $\tau \in(0, \varepsilon)$ (for $\varepsilon>0$ fixed). The key insight, as in [14, Section 5.2], is noticing that (5.4) strongly resembles a Riemann sum for the integral form for the mass-conserving AC flow from Theorem 3.7. To exploit this, we define the piecewise constant function $z_{\tau}:[0, \infty) \rightarrow \mathcal{V}$,

$$
z_{\tau}(s):= \begin{cases}e^{-\tau / \varepsilon} e^{\tau \Delta} \beta_{1}^{[\tau]}, & 0 \leqslant s \leqslant \tau, \\ e^{-k \tau / \varepsilon} e^{k \tau \Delta} \beta_{k}^{[\tau]}, & (k-1) \tau<s \leqslant k \tau \text { for } k \in \mathbb{N},\end{cases}
$$

and the function

$$
\gamma_{\tau}(s):=e^{s / \varepsilon} e^{-s \Delta} z_{\tau}(s)= \begin{cases}e^{-(\tau-s) / \varepsilon} e^{(\tau-s) \Delta} \beta_{1}^{[\tau]}, & 0 \leqslant s \leqslant \tau, \\ e^{-(k \tau-s) / \varepsilon} e^{(k \tau-s) \Delta} \beta_{k}^{[\tau]}, & (k-1) \tau<s \leqslant k \tau \text { for } k \in \mathbb{N} .\end{cases}
$$

We note an important convergence result.

Proposition 5.3 For any sequence $\tau_{n}^{\prime} \rightarrow 0$ with $\tau_{n}^{\prime}<\varepsilon$ for all $n$, there exists a function $z$ : $[0, \infty) \rightarrow \mathcal{V}$ and a subsequence $\tau_{n}$ of $\tau_{n}^{\prime}$ such that $z_{\tau_{n}}$ converges weakly to $z$ in $L_{\text {loc }}^{2}([0, \infty) ; \mathcal{V})$ and $z_{\tau_{n}}$ weak*-converges to $z$ in $L_{\text {loc }}^{\infty}([0, \infty) ; \mathcal{V})$.

Proof For $N \in \mathbb{N}$, consider $\left.z_{\tau}\right|_{[0, N]}$. As the $\beta_{k}^{[\tau]} \in \mathcal{V}_{[-1,1]}$ for all $k$ and $\tau$ by Lemma 5.1, we have for all $s \in[0, N]$ and $\tau<\varepsilon$

$$
\left\|z_{\tau}(s)\right\|_{\mathcal{V}} \leqslant \sup _{s^{\prime} \in[0, N+\varepsilon]}\left\|e^{-s^{\prime}\left(\frac{1}{\varepsilon} I-\Delta\right)}\right\| \cdot\|\mathbf{1}\|_{\mathcal{V}} \leqslant \max \left\{1, e^{(N+\varepsilon)\left(\|\Delta\|-\varepsilon^{-1}\right)}\right\} \cdot\|\mathbf{1}\|_{\mathcal{V}},
$$

where we have used that for $s \leqslant N$, the corresponding $k \tau$ in the exponent of $z_{\tau}(s)$ is less than $N+$ $\tau$ and that $\left\|e^{-s^{\prime}\left(\frac{1}{\varepsilon} I-\Delta\right)}\right\|=e^{s^{\prime}\left(\|\Delta\|-\varepsilon^{-1}\right)}$ is maximised at the endpoints of $[0, N+\varepsilon]$. Therefore, the $\left.z_{\tau}\right|_{[0, N]}$ are uniformly bounded in $\|\cdot\|_{\mathcal{V}}$ (and therefore in $\|\cdot\|_{\infty}$ since all norms on $\mathcal{V}$ are 
equivalent) for $\tau<\varepsilon$, and hence, they lie in a closed ball in $L^{2}([0, N] ; \mathcal{V})$ and in $L^{\infty}([0, N] ; \mathcal{V})$. By the Banach-Alaoglu theorem, the former ball is weak-compact and the latter ball is weak*compact. Hence, for any $\tau_{n}^{\prime} \downarrow 0$, there exists $\tau_{n}^{\prime \prime}$ a subsequence of $\tau_{n}^{\prime}$ and $z \in L^{2}([0, N]$; $\mathcal{V})$ and $w \in L^{\infty}([0, N] ; \mathcal{V})$ such that

$$
\left.z_{\tau_{n}^{\prime \prime}}\right|_{[0, N]} \rightarrow z \text { in } L^{2}([0, N] ; \mathcal{V}),\left.\quad \quad \quad z_{\tau_{n}^{\prime \prime}}\right|_{[0, N]} \rightarrow^{*} w \text { in } L^{\infty}([0, N] ; \mathcal{V}) .
$$

We claim that $z=w$ a.e. on $[0, N]$. By the definitions of the weak and weak* topologies, we have that for all $f \in L^{2}([0, N] ; \mathcal{V})$ and $g \in L^{1}([0, N] ; \mathcal{V})$

$$
\int_{0}^{N}\left\langle z_{\tau_{n}^{\prime \prime}}(t), f(t)\right\rangle_{\mathcal{V}} d t \rightarrow \int_{0}^{N}\langle z(t), f(t)\rangle_{\mathcal{V}} d t, \quad \int_{0}^{N}\left\langle z_{\tau_{n}^{\prime \prime}}(t), g(t)\right\rangle_{\mathcal{V}} d t \rightarrow \int_{0}^{N}\langle w(t), g(t)\rangle_{\mathcal{V}} d t
$$

Hence, for any $\mathcal{A} \subseteq[0, N]$ (measurable) and $i \in V$ consider $f(t):=\chi_{\mathcal{A}}(t) \chi_{i}$. Then $f \in$ $L^{2}([0, N] ; \mathcal{V}) \cap L^{1}([0, N] ; \mathcal{V})$ and so for all measurable $\mathcal{A} \subseteq[0, N]$,

$$
\int_{\mathcal{A}} z_{i}(t)-w_{i}(t) d t=0 .
$$

Hence, $z_{i}=w_{i}$ a.e. for each $i \in V$, so $z=w$ a.e. on $[0, N]$.

Finally, we extend to $[0, \infty)$ by a 'local-to-global' diagonal argument. First, we take $N=1$ : by above we can choose a subsequence $\tau^{(1)}$ of $\tau^{\prime}$ such that $z_{\tau_{n}^{(1)}}$ converges in both the weak topology on $L^{2}$ and the weak* topology on $L^{\infty}$ to some $z$ on $[0,1]$. Then to move from $N$ to $N+1$, we likewise choose a subsequence $\tau^{(N+1)}$ of $\tau^{(N)}$ such that $z_{\tau_{n}^{(N+1)}}$ converges in both senses to $z$ on $[0, N+1]$. Finally, define $\tau_{n}:=\tau_{n}^{(n)}$. Then for all bounded $T \subseteq[0, \infty)$, we have $T \subseteq[0, M]$ for some $M \in \mathbb{N}$ and hence $\left.z_{\tau_{n}}\right|_{T}$ is eventually a subsequence of $\left.z_{\tau_{n}^{(M)}}\right|_{T}$ and so converges in both senses to $\left.z\right|_{T}$.

Corollary 5.4 From $z_{\tau_{n}} \rightarrow z$ in $L_{\text {loc }}^{2}([0, \infty) ; \mathcal{V})$, we infer:

A. $\gamma_{\tau_{n}} \rightarrow \gamma$, where $\gamma(s):=e^{s / \varepsilon} e^{-s \Delta} z$, and $z_{\tau_{n}}-\bar{z}_{\tau_{n}} \mathbf{1} \rightarrow z-\bar{z} \mathbf{1}\left(\right.$ both in $L_{l o c}^{2}([0, \infty) ; \mathcal{V})$ ).

B. For all $t \geqslant 0$,

$$
\int_{0}^{t} z_{\tau_{n}}(s)-\bar{z}_{\tau_{n}}(s) \mathbf{1} d s \rightarrow \int_{0}^{t} z(s)-\bar{z}(s) \mathbf{1} d s .
$$

C. Replacing $\tau_{n}$ by an appropriate subsequence, we have strong convergence of the Cesàro sums, i.e. for all bounded $T \subseteq[0, \infty)$

$$
\frac{1}{N} \sum_{n=1}^{N} z_{\tau_{n}} \rightarrow z \quad \text { and } \quad \frac{1}{N} \sum_{n=1}^{N} \gamma_{\tau_{n}} \rightarrow \gamma \quad \text { in } L^{2}(T ; \mathcal{V})
$$

as $N \rightarrow \infty$.

And from $z_{\tau_{n}} \rightarrow^{*} z$ in $L_{l o c}^{\infty}([0, \infty) ; \mathcal{V})$, we infer:

D. $\gamma_{\tau_{n}} \rightarrow^{*} \gamma$ in $L_{l o c}^{\infty}([0, \infty) ; \mathcal{V})$.

Proof Claim (A) follows since $f \mapsto e^{s / \varepsilon} e^{-s \Delta} f$ (where $s$ is the argument of $f$ ) and $f \mapsto f-\bar{f} \mathbf{1}$ are continuous self-adjoint maps on $L^{2}(T ; \mathcal{V})$ for $T$ bounded. Hence for all $f \in L^{2}(T ; \mathcal{V})$,

$$
\left(\gamma_{\tau_{n}}, f\right)_{s \in T}=\left(z_{\tau_{n}}, e^{s / \varepsilon} e^{-s \Delta} f\right)_{s \in T} \rightarrow\left(z, e^{s / \varepsilon} e^{-s \Delta} f\right)_{s \in T}=(\gamma, f)_{s \in T}
$$


and

$$
\left(z_{\tau_{n}}-\bar{z}_{\tau_{n}} \mathbf{1}, f\right)_{t \in T}=\left(z_{\tau_{n}}, f-\bar{f} \mathbf{1}\right)_{t \in T} \rightarrow(z, f-\bar{f} \mathbf{1})_{t \in T}=(z-\bar{z} \mathbf{1}, f)_{t \in T}
$$

Claim (B) is a direct consequence of weak convergence. Claim (C) follows by the BanachSaks theorem [4], which states that weak $L^{p}$ convergence on a bounded interval entails strong convergence of Cesàro sums on that interval along an appropriate subsequence, and a 'local-toglobal' diagonal argument as in the above proof to extract a subsequence that works on all of $[0, \infty)$. Claim (D) follows since $f \mapsto e^{s / \varepsilon} e^{-s \Delta} f$ is continuous on $L^{\infty}(T ; \mathcal{V})$ and on $L^{1}(T ; \mathcal{V})$, for $T$ bounded, and with respect to the pairing of $L^{\infty}$ with $L^{1}$, for all $f \in L^{\infty}(T ; \mathcal{V})$ and $g \in L^{1}(T ; \mathcal{V})$

$$
\int_{T}\left\langle e^{s / \varepsilon} e^{-s \Delta} f(s), g(s)\right\rangle_{\mathcal{V}} d s=\int_{T}\left\langle f(s), e^{s / \varepsilon} e^{-s \Delta} g(s)\right\rangle_{\mathcal{V}} d s
$$

so the map is 'self-adjoint' and so (D) follows by the same argument as (A).

We now return to the question of convergence of the semi-discrete iterates. Taking $\tau$ to zero along the sequence $\tau_{n}$, we define for all $t \geqslant 0$ the continuum-time function

$$
\hat{u}(t):=\lim _{n \rightarrow \infty, m=\left\lceil t / \tau_{n}\right\rceil} u_{m}^{\left[\tau_{n}\right]} .
$$

Therefore by (5.4) (note that $m$ depends on both $t$ and $n$, but for the sake of readability, we will write $m$ rather than $\left.m_{n}(t)\right)$

$$
\begin{aligned}
\hat{u}(t)=\bar{u} \mathbf{1}+\lim _{n \rightarrow \infty}\left(e^{m \tau_{n} / \varepsilon} e^{-m \tau_{n} \Delta}\left(u_{0}-\bar{u} \mathbf{1}\right)\right. & \\
& \left.+\frac{1}{\varepsilon} e^{m \tau_{n} / \varepsilon} e^{-m \tau_{n} \Delta} \tau_{n} \sum_{k=1}^{m} e^{-k \tau_{n} / \varepsilon} e^{k \tau_{n} \Delta}\left(\beta_{k}^{\left[\tau_{n}\right]}-\bar{\beta}_{k}^{\left[\tau_{n}\right]} \mathbf{1}\right)\right)
\end{aligned}
$$

and by rewriting the sum term via the definition of $z_{\tau_{n}}$ :

$$
\hat{u}(t)=\bar{u} \mathbf{1}+\lim _{n \rightarrow \infty} e^{m \tau_{n} / \varepsilon} e^{-m \tau_{n} \Delta}\left(u_{0}-\bar{u} \mathbf{1}\right)+\frac{1}{\varepsilon} e^{m \tau_{n} / \varepsilon} e^{-m \tau_{n} \Delta} \int_{0}^{m \tau_{n}} z_{\tau_{n}}(s)-\bar{z}_{\tau_{n}}(s) \mathbf{1} d s .
$$

We seek to show that the pair $(\hat{u}, \gamma)$ solves (3.7), i.e. $\hat{u}$ is an AC flow trajectory. We will do this by checking that $(\hat{u}, \gamma)$ satisfy the sufficient conditions given for $(u, \beta)$ in Theorem 3.7. We will spit this into two lemmas. First, we show all but one of the required conditions.

Proposition 5.5 The pair $(\hat{u}, \gamma)$ obeys:

(i) For all $t \geqslant 0, \hat{u}(t)$ exists and is given by

$$
\hat{u}(t)=\bar{u} \mathbf{1}+e^{t / \varepsilon} e^{-t \Delta}\left(u_{0}-\bar{u} \mathbf{1}\right)+\frac{1}{\varepsilon} e^{t / \varepsilon} e^{-t \Delta} \int_{0}^{t} e^{-s / \varepsilon} e^{s \Delta}(\gamma(s)-\bar{\gamma}(s) \mathbf{1}) d s .
$$

(ii) $\hat{u}(t) \in \mathcal{V}_{[0,1]}$ for all $t \geqslant 0$.

(iii) $\gamma-\bar{\gamma} \mathbf{1}$ is locally essentially bounded and locally integrable.

Proof Let $A:=\varepsilon^{-1} I-\Delta$ and $e_{n}:=m \tau_{n}-t \in\left[0, \tau_{n}\right)$. 
(i) Note that

$$
e^{m \tau_{n} / \varepsilon} e^{-m \tau_{n} \Delta}=e^{\left(t+e_{n}\right) A}=e^{t A}\left(I+\mathcal{O}\left(e_{n}\right)\right)=e^{t A}+\mathcal{O}\left(\tau_{n}\right)
$$

and so

$$
\begin{aligned}
\hat{u}(t)=\bar{u} \mathbf{1}+ & \lim _{n \rightarrow \infty} e^{t A}\left(u_{0}-\bar{u} \mathbf{1}\right) \\
& +\frac{1}{\varepsilon}\left(e^{t A}+\mathcal{O}\left(\tau_{n}\right)\right)\left(\int_{0}^{t} z_{\tau_{n}}(s)-\bar{z}_{\tau_{n}}(s) \mathbf{1} d s+\int_{t}^{t+e_{n}} z_{\tau_{n}}(s)-\bar{z}_{\tau_{n}}(s) \mathbf{1} d s\right) .
\end{aligned}
$$

Hence, as by Proposition 5.3, the $z_{\tau_{n}}-\bar{z}_{\tau_{n}} 1$ are uniformly bounded on any compact interval (and so on $\left[0, t+\max _{n} e_{n}\right]$ ), it follows by Corollary 5.4(B) that

$$
\hat{u}(t)=\bar{u} \mathbf{1}+e^{t / \varepsilon} e^{-t \Delta}\left(u_{0}-\bar{u} \mathbf{1}\right)+\frac{1}{\varepsilon} e^{t / \varepsilon} e^{-t \Delta} \int_{0}^{t} z(s)-\bar{z}(s) \mathbf{1} d s .
$$

Then, (5.6) follows immediately by Corollary 5.4(A).

(ii) $\hat{u}(t)$ is a limit of semi-discrete iterates, each of which lies in $\mathcal{V}_{[0,1]}$.

(iii) Since $\gamma-\bar{\gamma} \mathbf{1}$ is a weak limit of locally bounded and locally integrable functions, we have that $\gamma-\bar{\gamma} \mathbf{1}$ is locally bounded a.e. and is locally integrable.

Lastly, we check the sub-differential condition.

Lemma $5.6 \gamma(t) \in \mathcal{B}(\hat{u}(t))$ for a.e. $t \geqslant 0$.

We give two proofs of this result. We first recap part of the proof in [14] in order to derive the characterisation of $\gamma$ in (5.7), and next we give a novel proof of this result.

Proof (A), cf. $[14$, p. 4125]. By Corollary 5.4(C), on each bounded $T \subseteq[0, \infty), \gamma$ is the $L^{2}(T ; \mathcal{V})$ limit of

$$
S_{N}:=\frac{1}{N} \sum_{n=1}^{N} \gamma_{\tau_{n}}
$$

as $N \rightarrow \infty$. As $L^{2}$ convergence implies a.e. pointwise convergence along a subsequence, by a 'local-to-global' diagonal argument, there exists a sequence $N_{k} \rightarrow \infty$ such that for a.e. $t \geqslant 0$

$$
\gamma(t)=\lim _{k \rightarrow \infty} \frac{1}{N_{k}} \sum_{n=1}^{N_{k}} \gamma_{\tau_{n}}(t)
$$

Recall $A:=\varepsilon^{-1} I-\Delta, m:=\left\lceil t / \tau_{n}\right\rceil$, and $e_{n}:=m \tau_{n}-t \in\left[0, \tau_{n}\right)$. Then, by Lemma 5.1

$$
\begin{aligned}
\left\|\gamma_{\tau_{n}}(t)-\beta_{m}^{\left[\tau_{n}\right]}\right\| \mathcal{V}=\left\|\left(e^{-e_{n} A}-I\right) \beta_{m}^{\left[\tau_{n}\right]}\right\| \mathcal{V} & \leqslant\left(1-e^{-e_{n}\|A\| \mid}\right)\|\mathbf{1}\| \mathcal{V} \\
& <\left(1-e^{-\tau_{n}\|A\| \mid}\right)\|\mathbf{1}\|_{\mathcal{V}}<\tau_{n}\|A\|\|\mathbf{1}\| \mathcal{V} .
\end{aligned}
$$

Therefore, for a.e. $t \geqslant 0$,

$$
\left\|\gamma(t)-\frac{1}{N_{k}} \sum_{n=1}^{N_{k}} \beta_{m}^{\left[\tau_{n}\right]}\right\|_{\mathcal{V}} \leqslant\left\|\gamma(t)-\frac{1}{N_{k}} \sum_{n=1}^{N_{k}} \gamma_{\tau_{n}}(t)\right\|_{\mathcal{V}}+\|A\|\|\mathbf{1}\| \mathcal{V} \frac{1}{N_{k}} \sum_{n=1}^{N_{k}} \tau_{n} \rightarrow 0
$$


as $k \rightarrow \infty$ (since $\tau_{n} \rightarrow 0$ and the convergence of a sequence implies the convergence of its Cesàro sums to the same limit), so for a.e. $t \geqslant 0$

$$
\gamma(t)=\lim _{k \rightarrow \infty} \frac{1}{N_{k}} \sum_{n=1}^{N_{k}} \beta_{m}^{\left[\tau_{n}\right]} .
$$

The result then follows identically as in [14, p. 4125] - we here omit the details.

Proof (B). Fix $i \in V$ and bounded $T \subseteq[0, \infty)$. For tidyness of notation, we define $x_{n}(t):=u_{\left\lceil t / \tau_{n}\right\rceil}^{\left[\tau_{n}\right]}$ and $x(t):=\hat{u}_{i}(t)$, and likewise $\xi_{n}(t):=\left(\beta_{\left[t / \tau_{n}\right]}^{\left[\tau_{n}\right]}\right)_{i}$ and $\xi(t):=\gamma_{i}(t)$. Let

$$
T_{1}:=\{t \in T \mid x(t)=0\}, \quad T_{2}:=\{t \in T \mid x(t) \in(0,1)\}, \quad T_{3}:=\{t \in T \mid x(t)=1\} .
$$

Then, it suffices to show that $\xi \geqslant 0$ a.e. on $T_{1}, \xi=0$ a.e. on $T_{2}$ and $\xi \leqslant 0$ a.e. on $T_{3}$.

By Corollary 5.4(D), we have that $\left(\gamma_{\tau_{n}}\right)_{i} \rightarrow^{*} \xi$ in $L_{l o c}^{\infty}([0, \infty) ; \mathcal{V})$ and therefore $\left(\gamma_{\tau_{n}}\right)_{i} \rightarrow^{*} \xi$ in $L^{\infty}(T, \mathbb{R})$, i.e. for all $f \in L^{1}(T, \mathbb{R})$, as $n \rightarrow \infty$

$$
\int_{T}\left(\gamma_{\tau_{n}}\right)_{i}(t) f(t) d t \rightarrow \int_{T} \xi(t) f(t) d t .
$$

Recalling from Proof (A) that $\left(\gamma_{\tau_{n}}\right)_{i}(t)=\xi_{n}(t)+\mathcal{O}\left(\tau_{n}\right)$, we infer that as $n \rightarrow \infty$

$$
\int_{T} \xi_{n}(t) f(t) d t \rightarrow \int_{T} \xi(t) f(t) d t .
$$

By (5.5), we have by definition that for all $t \in T_{1}, x_{n}(t) \rightarrow 0$. We define the (measurable) sets $A_{N}:=\left\{t \in T_{1} \mid \forall n \geqslant N x_{n}(t)<1 / 2\right\}$. Then, by the pointwise convergence of the $x_{n}, T_{1}=\bigcup_{N} A_{N}$. Suppose for contradiction that for some $X \subseteq T_{1}$ of positive measure, $\xi<0$ on $X$. So there exists $\delta>0$ and $Y \subseteq X$ of positive measure such that $\xi \leqslant-\delta$ on $Y$. As $T_{1}$ is the union of the $A_{N}$, there exists $N \in \mathbb{N}$ such that $Y \cap A_{N}$ is of positive measure. Taking test function $f=\chi_{Y \cap A_{N}}$, we infer that as $n \rightarrow \infty$ (and $\mu$ the Lebesgue measure)

$$
\int_{Y \cap A_{N}} \xi_{n}(t) d t \rightarrow \int_{Y \cap A_{N}} \xi(t) d t \leqslant-\delta \mu\left(Y \cap A_{N}\right)<0
$$

but since $\beta_{\left[t / \tau_{n}\right\rceil}^{\left[\tau_{n}\right]} \in \mathcal{B}\left(u_{\left\lceil t / \tau_{n}\right\rceil}^{\left[\tau_{n}\right]}\right)$, we have that if $t \in A_{N}$, then for all $n \geqslant N, \xi_{n}(t) \geqslant 0$, so this is a contradiction. Hence, $\xi \geqslant 0$ a.e. on $T_{1}$. By the same argument, $\xi \leqslant 0$ a.e. on $T_{3}$.

Finally, for all $t \in T_{2}$, since $x_{n}(t) \rightarrow x(t), x_{n}(t)$ is eventually in $(0,1)$. Define $B_{N}:=\left\{t \in T_{2} \mid\right.$ $\left.\forall n \geqslant N x_{n}(t) \in(0,1)\right\}$, and note that $T_{2}=\bigcup_{N} B_{N}$ and that for $t \in B_{N}$ and $n \geqslant N, \xi_{n}(t)=0$ since $\beta_{\left\lceil t / \tau_{n}\right\rceil}^{\left[\tau_{n}\right]} \in \mathcal{B}\left(u_{\left\lceil t / \tau_{n}\right\rceil}^{\left[\tau_{n}\right]}\right)$. Suppose for contradiction that for some $X \subseteq T_{2}$ of positive measure, $\xi \neq 0$ on $X$. Then WLOG, there exists $\delta>0$ and $Y \subseteq X$ of positive measure such that $\xi \geqslant \delta$ on $Y$. As before there exists $N \in \mathbb{N}$ such that $Y \cap B_{N}$ is of positive measure. Taking $f=\chi_{Y \cap B_{N}}$, we infer that as $n \rightarrow \infty$ (for $n \geqslant N)$

$$
0=\int_{Y \cap B_{N}} \xi_{n}(t) d t \rightarrow \int_{Y \cap B_{N}} \xi(t) d t \geqslant \delta \mu\left(Y \cap A_{N}\right)>0
$$

a contradiction. Therefore, $\xi=0$ a.e. on $T_{2}$.

Note Proof $(B)$ is also valid in the non-mass-conserving case. We thank Dr Carolin Kreisbeck for her suggestion of using weak* $L^{\infty}$ convergence which led to the development of this proof. 
Therefore, by Theorem 3.7, we have the following convergence result.

Theorem 5.7 (Cf. [14, Theorem 5.4]) For any given $u_{0} \in \mathcal{V}_{[0,1]} \backslash\{\mathbf{0}, \mathbf{1}\}, \varepsilon>0$ and $\tau_{n} \downarrow 0$, there exists a subsequence $\tau_{n}^{\prime}$ of $\tau_{n}$ with $\tau_{n}^{\prime}<\varepsilon$ for all $n$, such that along this subsequence, the semidiscrete iterates $\left(u_{m}^{\left[\tau_{n}^{\prime}\right]}, \beta_{m}^{\left[\tau_{n}^{\prime}\right]}\right)$ given by (4.1) with initial state $u_{0}$ converge to an AC solution. That $i$, there exists $(\hat{u}, \gamma)$ a solution to $(3.7)$ with $\hat{u}(0)=u_{0}$, such that:

- $\beta_{\left\lceil\cdot / \tau_{n}^{\prime}\right\rceil}^{\left[\tau_{n}^{\prime}\right]}$ converges to $\gamma$ weakly in $L_{\text {loc }}^{2}([0, \infty) ; \mathcal{V})$ and weakly* in $L_{l o c}^{\infty}([0, \infty) ; \mathcal{V})$,

- for each $t \geqslant 0$ and for $m:=\left\lceil t / \tau_{n}^{\prime}\right\rceil, u_{m}^{\left[\tau_{n}^{\prime}\right]} \rightarrow \hat{u}(t)$ as $n \rightarrow \infty$, and

- there is a sequence $N_{k} \rightarrow \infty$ such that for almost every $t \geqslant 0, \frac{1}{N_{k}} \sum_{n=1}^{N_{k}} \beta_{m}^{\left[\tau_{n}^{\prime}\right]} \rightarrow \gamma(t)$ as $k \rightarrow \infty$.

Note This result proves Theorem 3.9, i.e. the existence of AC solutions.

Note For $u_{0}=\mathbf{0}$ or $\mathbf{1}$, the $u_{m}^{\left[\tau_{n}\right]} \equiv u_{0}$ trivially converge but the $\beta_{m}^{\left[\tau_{n}\right]}$ need not converge.

Note As in [14, Remark 5.5], we can avoid passing to a subsequence in all but the last of these convergences because of Theorem 3.8. Recall the fact noted in [14]: if $(X, \rho)$ is a topological space, $x_{n}, x \in X$, and every subsequence of $x_{n}$ has a further subsequence converging to $x$ in $\rho$, then $x_{n} \rightarrow x$ in $\rho$. Let $\tau_{n} \downarrow 0$, with $\tau_{n}<\varepsilon$ for all $n$, and $x_{n}:=t \mapsto u_{\left\lceil t / \tau_{n}\right\rceil}^{\left[\tau_{n}\right]} \in\left(\mathcal{V}_{t \in[0, \infty)}, \rho\right)$ for $\rho$ the topology of pointwise convergence. By Theorem 5.7 applied to $\tau_{n_{k}}$, every subsequence $x_{n_{k}}$ has a subsequence converging to an AC solution with initial condition $u_{0}$. By uniqueness, these must equal $\hat{u}$. Therefore, $x_{n} \rightarrow \hat{u}$ pointwise, without passing to a subsequence. Likewise, the corresponding $\gamma-\bar{\gamma} \mathbf{1}$ is unique up to a.e. equivalence, so $z_{\tau_{n}}-\overline{z_{\tau_{n}}} \mathbf{1} \rightarrow z-\bar{z} \mathbf{1}$ and $\gamma_{\tau_{n}}-\overline{\gamma_{\tau_{n}}} \mathbf{1} \rightarrow$ $\gamma-\bar{\gamma} \mathbf{1}$ in $L_{l o c}^{2}([0, \infty ; \mathcal{V})$ without passing to a subsequence. Finally, when $\gamma$ is unique up to a.e. equivalence (i.e. when $\hat{u}(t) \notin \mathcal{V}_{\{0,1\}}$ for all $\left.t \geqslant 0\right)$, then $\gamma_{\tau_{n}} \rightarrow \gamma$ in $L_{\text {loc }}^{2}\left([0, \infty ; \mathcal{V})\right.$ and $\gamma_{\tau_{n}} \rightarrow^{*} \gamma$ in $L_{l o c}^{\infty}([0, \infty ; \mathcal{V})$ without passing to a subsequence.

\subsection{Consequences of Theorem 5.7}

Given this representation of the unique solution to (3.7) as a limit of semi-discrete approximations, we can deduce a number of properties of this solution.

First, following [14, Section 5.3], we verify that the unique AC solution is a decreasing flow of $\mathrm{GL}_{\varepsilon}$ by considering the Lyapunov functional $H$ for the semi-discrete scheme defined in (4.31), and in doing so obtain a control on the behaviour of $\mathrm{GL}_{\varepsilon}(\hat{u}(t))$.

We recall from [14], for $u \in \mathcal{V}_{[0,1]}$, the following scaling of the Lyapunov functional

$$
H_{\tau}(u):=\frac{1}{2 \tau} H(u)=\mathrm{GL}_{\varepsilon}(u)-\frac{1}{2} \tau\left\langle u, Q_{\tau} u\right\rangle_{\mathcal{V}},
$$

where $\tau^{2} Q_{\tau}:=e^{-\tau \Delta}-I+\tau \Delta$. Furthermore, we recall the result.

Proposition 5.8 ([14, Proposition 5.6]) Let $u_{\tau}, u \in \mathcal{V}_{[0,1]}$ satisfy $\left\|u_{\tau}-u\right\|_{\mathcal{V}} \rightarrow 0$ as $\tau \rightarrow 0$. Then it follows that $H_{\tau}\left(u_{\tau}\right) \rightarrow \mathrm{GL}_{\varepsilon}(u)$. 
Theorem 5.9 (Cf. [14, Theorem 5.7]) The AC trajectory $\hat{u}$ defined by (5.5) has $\mathrm{GL}_{\varepsilon}(\hat{u}(t))$ monotonically decreasing in $t$. More precisely: for all $t>s \geqslant 0$,

$$
\mathrm{GL}_{\varepsilon}(\hat{u}(s))-\mathrm{GL}_{\varepsilon}(\hat{u}(t)) \geqslant \frac{1}{2(t-s)}\|\hat{u}(s)-\hat{u}(t)\|_{\mathcal{V}}^{2} .
$$

Proof We reproduce the proof from [14]. Let $t>s \geqslant 0$ and $m:=\left\lceil s / \tau_{n}\right\rceil$ and $\ell:=\left\lceil t / \tau_{n}\right\rceil$. Next, note a standard inner product space fact: for all sequences $v_{n} \in \mathcal{V}$,

$$
\sum_{n=1}^{N}\left\|v_{n}\right\|_{\mathcal{V}}^{2}=\frac{1}{N}\left\|\sum_{n=1}^{N} v_{n}\right\|_{\mathcal{V}}^{2}+\frac{1}{N} \sum_{k<n}\left\|v_{n}-v_{k}\right\|_{\mathcal{V}}^{2} \geqslant \frac{1}{N}\left\|\sum_{n=1}^{N} v_{n}\right\|_{\mathcal{V}}^{2}
$$

Now by (5.5), we have $u_{m}^{\left[\tau_{n}\right]} \rightarrow \hat{u}(s)$ and $u_{\ell}^{\left[\tau_{n}\right]} \rightarrow \hat{u}(t)$. It follows that:

$$
\begin{array}{rlrl}
\mathrm{GL}_{\varepsilon}(\hat{u}(s))-\mathrm{GL}_{\varepsilon}(\hat{u}(t)) & =\lim _{n \rightarrow \infty} H_{\tau_{n}}\left(u_{m}^{\left[\tau_{n}\right]}\right)-H_{\tau_{n}}\left(u_{\ell}^{\left[\tau_{n}\right]}\right) & \text { by Proposition } 5.8 \\
& \geqslant \lim _{n \rightarrow \infty} \frac{1}{2 \tau_{n}}\left(1-\frac{\tau_{n}}{\varepsilon}\right) \sum_{k=m}^{\ell-1}\left\|u_{k+1}^{\left[\tau_{n}\right]}-u_{k}^{\left[\tau_{n}\right]}\right\|_{\mathcal{V}}^{2} \quad \text { by (4.32) } \\
& \geqslant \lim _{n \rightarrow \infty} \frac{1}{2 \tau_{n}}\left(1-\frac{\tau_{n}}{\varepsilon}\right) \frac{1}{\ell-m}\left\|u_{\ell}^{\left[\tau_{n}\right]}-u_{m}^{\left[\tau_{n}\right]}\right\|_{\mathcal{V}}^{2} \quad \text { by (5.9) } \\
& =\frac{1}{2(t-s)}\|\hat{u}(s)-\hat{u}(t)\|_{\mathcal{V}}^{2} \geqslant 0
\end{array}
$$

as desired, since $\tau_{n}(\ell-m) \rightarrow t-s$.

Note Since $\mathrm{GL}_{\varepsilon}(\hat{u}(s))-\mathrm{GL}_{\varepsilon}(\hat{u}(t)) \leqslant \mathrm{GL}_{\varepsilon}(\hat{u}(s)) \leqslant \mathrm{GL}_{\varepsilon}(\hat{u}(0))$, it follows by (5.8) that

$$
\|\hat{u}(s)-\hat{u}(t)\|_{\mathcal{V}} \leqslant \sqrt{|t-s|} \sqrt{2 \mathrm{GL}_{\varepsilon}(\hat{u}(0))}
$$

which as in [14, Remark 5.8] gives an explicit $C^{0,1 / 2}$ condition for $\hat{u}$.

Next, we derive some controls on $\gamma$ and thereby infer a Lipschitz condition on $\hat{u}$.

Lemma 5.10 For $\gamma(t)$ given at a.e. $t \in T$ by (5.7), at each such $t$

$$
\gamma(t)-\bar{\gamma}(t) \mathbf{1} \in \mathcal{V}_{[\bar{u}-1, \bar{u}]} \text { and } \gamma(t) \in \mathcal{V}_{[-1,1]}
$$

Proof Follows immediately from (5.7) and the controls in Lemma 5.1.

Theorem 5.11 The AC trajectory $\hat{u}$ defined by $(5.5)$ has $\hat{u} \in C^{0,1}([0, \infty) ; \mathcal{V})$.

Proof By (3.12) and the argument in [14, Theorem 3.13], we have for $t_{1}<t_{2}$

$$
\hat{u}\left(t_{2}\right)-\hat{u}\left(t_{1}\right)=\left(e^{\left(t_{2}-t_{1}\right) A}-I\right)\left(\hat{u}\left(t_{1}\right)-\bar{u} \mathbf{1}\right)+\frac{1}{\varepsilon} \int_{0}^{t_{2}-t_{1}} e^{s A}\left(\gamma\left(t_{2}-s\right)-\bar{\gamma}\left(t_{2}-s\right) \mathbf{1}\right) d s,
$$


where $A:=\varepsilon^{-1} I-\Delta$ and $\gamma(t)$ is given at a.e. $t \geqslant 0$ by (5.7). By Lemma 5.10, $\gamma(t)-\bar{\gamma}(t) \mathbf{1} \in$ $\mathcal{V}_{[\bar{u}-1, \bar{u}]}$ for a.e. $t \geqslant 0$, and

$$
\|\hat{u}(t)-\bar{u} \mathbf{1}\|_{\mathcal{V}} \leqslant \max \{\bar{u}, 1-\bar{u}\}\|\mathbf{1}\| \mathcal{V}=: \rho\|\mathbf{1}\|_{\mathcal{V}}
$$

since $\hat{u}(t) \in \mathcal{V}_{[0,1]}$ for all $t \geqslant 0$. Let $B_{\delta t}:=\left(e^{\delta t A}-I\right) / \delta t$. Then, $\left\|B_{\delta t}\right\|=\left(e^{\delta t / \varepsilon}-1\right) / \delta t$, which monotonically increases in $\delta t$. We thus have for $t_{2}-t_{1}<1$,

$$
\begin{aligned}
\frac{\left\|\hat{u}\left(t_{2}\right)-\hat{u}\left(t_{1}\right)\right\|_{\mathcal{V}}}{t_{2}-t_{1}} & \leqslant\left\|B_{t_{2}-t_{1}}\right\| \cdot \rho\|\mathbf{1}\| \mathcal{V}+\frac{1}{\varepsilon} \operatorname{ess}_{s \in\left[0, t_{2}-t_{1}\right]}\left\|e^{s A}\left(\gamma\left(t_{2}-s\right)-\bar{\gamma}\left(t_{2}-s\right) \mathbf{1}\right)\right\|_{\mathcal{V}} \\
& \leqslant \frac{e^{\left(t_{2}-t_{1}\right) / \varepsilon}-1}{t_{2}-t_{1}} \cdot \rho\|\mathbf{1}\|_{\mathcal{V}}+\frac{1}{\varepsilon} \sup _{s \in\left[0, t_{2}-t_{1}\right]}\left\|e^{s A}\right\| \cdot \rho\|\mathbf{1}\|_{\mathcal{V}} \\
& \leqslant \frac{e^{\left(t_{2}-t_{1}\right) / \varepsilon}-1}{t_{2}-t_{1}} \cdot \rho\|\mathbf{1}\| \mathcal{V}+\frac{1}{\varepsilon} e^{\left(t_{2}-t_{1}\right) / \varepsilon} \cdot \rho\|\mathbf{1}\|_{\mathcal{V}} \\
& <\rho\|\mathbf{1}\|_{\mathcal{V}}\left(e^{1 / \varepsilon}-1+\frac{1}{\varepsilon} e^{1 / \varepsilon}\right)
\end{aligned}
$$

and for $t_{2}-t_{1} \geqslant 1$ we have

$$
\frac{\left\|\hat{u}\left(t_{2}\right)-\hat{u}\left(t_{1}\right)\right\|_{\mathcal{V}}}{t_{2}-t_{1}} \leqslant\left\|\hat{u}\left(t_{2}\right)-\hat{u}\left(t_{1}\right)\right\|_{\mathcal{V}} \leqslant\|\mathbf{1}\|_{\mathcal{V}}
$$

completing the proof.

\section{Conclusion}

In this paper, we have translated Rubinstein and Sternberg's mass-conserving Allen-Cahn flow [34] into the context of dynamics on graphs and have proved existence, uniqueness and regularity properties of the resulting differential equation with a double-obstacle potential. Following [14], we have formulated a semi-discrete scheme for mass-conserving graph Allen-Cahn flow, proved that the mass-conserving graph MBO scheme emerges exactly as the $\lambda=1$ special case of this semi-discrete scheme and shown that the Lyapunov functional from [14] remains a Lyapunov functional in the mass-conserving case.

Using the tools of convex optimisation, we have characterised the solutions of this massconserving semi-discrete scheme, allowing us to prove that:

- As $\lambda \uparrow 1$, the semi-discrete solutions for a given $\lambda$ converge in $\mathcal{V}$ to a solution for $\lambda=1$, yielding a choice function for MBO solutions.

- As $\tau, \lambda \downarrow 0$ (and $\varepsilon$ fixed) the semi-discrete solutions converge pointwise to the Allen-Cahn solution.

Additionally, we have extended some results from [14] and [38] concerning pinning/freezing to the mass-conserving case, observing remarkably that the same sufficient conditions for pinning hold as in the non-mass-conserving case.

In future work, we seek to devise a formulation of mass-conserving multi-class Allen-Cahn flow on graphs (with a 'multi-obstacle potential') and extend the results of this paper to the multi-class case, thereby giving a choice function for the multi-class MBO scheme which selects 
the $\lambda \uparrow 1$ limit of the multi-class semi-discrete solutions. We shall then compare this method for solving the multi-class MBO scheme on graphs with others in the literature, e.g. the auction dynamics of Jacobs, Merkurjev, and Esedoḡlu [26].

\section{Acknowledgements}

This project has received funding from the European Union's Horizon 2020 research and innovation programme under the Marie Skłodowska-Curie grant agreement No 777826. Thanks to Dr. Carolin Kreisbeck for her suggestion of using weak ${ }^{*} L^{\infty}$ convergence for an alternative method for proving the convergence of the semi-discrete iterates.

\section{Conflicts of interest}

None.

\section{References}

[1] Acikmese, B. (2015) Spectrum of laplacians for graphs with self-loops. arXiv preprint arXiv:1505.08133.

[2] Ambrosio, L., Gigli, L. \& Savaré, G. (2008) Gradient Flows in Metric Spaces and in the Space of Probability Measures, 2nd ed. Lectures in Mathematics ETH Zürich, Birkhäuser Verlag, Basel.

[3] BAE, E. \& MERKuRJev, E. (2017) Convex variational methods on graphs for multiclass segmentation of high-dimensional data and point clouds. J. Math. Imaging Vis. 58, 468-493.

[4] BANACH, S. \& SAKS, S. (1930) Sur la convergence dans les champs $L^{p}$. Studia Mathematica 2, 51-57.

[5] Bence, J., Merriman, B. \& Osher, S. (1992) Diffusion Generated Motion by Mean Curvature. CAM Report, 92-18, Department of Mathematics, University of California, Los Angeles.

[6] Bertozzi, A. L. \& Flenner, A. (2012) Diffuse interface models on graphs for analysis of high dimensional data. Multiscale Model. Simul. 10(3), 1090-1118.

[7] Blowey, J. F. \& Elliott, C. M. (1991) The Cahn-Hilliard gradient theory for phase separation with non-smooth free energy, Part I: mathematical analysis. Eur. J. Appl. Math. 3, 233-279.

[8] Blowey, J. F. \& ElliotT, C. M. (1992) The Cahn-Hilliard gradient theory for phase separation with non-smooth free energy, Part II: numerical analysis. Eur. J. Appl. Math 3, 147-179.

[9] Blowey, J. F. \& ElliotT, C. M. (1993) Curvature dependent phase boundary motion and parabolic double obstacle problems. In: W. M. Ni, L. A. Peletier and J. L. Vazquez (editors), Degenerate Diffusions. The IMA Volumes in Mathematics and its Applications, Vol. 47, pp. 19-60.

[10] Bosch, J., Klamt, S. \& Stoll, M. (2018) Generalizing diffuse interface methods on graphs: nonsmooth potentials and hypergraphs. SIAM J. Appl. Math. 78(3), 1350-1377.

[11] Boyd, S. \& Vandenberghe, L. (2004) Convex Optimization, Cambridge University Press, Cambridge, UK.

[12] Brezis, H. (2010) Functional Analysis, Sobolev Spaces and Partial Differential Equations, Springer, Berlin.

[13] Bronsard, L. \& Kohn, R. V. (1991) Motion by mean curvature as the singular limit of GinzburgLandau dynamics. J. Differ. Equations 90, 211-237.

[14] Budd, J. \& VAN GENNIP, Y. (2020) Graph Merriman-Bence-Osher as a SemiDiscrete implicit Euler scheme for graph Allen-Cahn flow. SIAM J. Math. Anal. 52(5), 4101-4139.

[15] BUdD, J., VAN GENNIP, Y. \& LATZ, J. (2021) Classification and image processing with a semidiscrete scheme for fidelity forced Allen-Cahn on graphs. GAMM Mitteilungen 44(1), e202100004.

[16] CAHn, J. W. (1964) On spinodal decomposition. Acta Metall. 9, 795-801.

[17] CAhn, J. W. \& Hilliard, J. E. (1958) Free energy of a nonuniform system. I. Interfacial free energy. J. Chem. Phys. 28(2), 258-267. 
[18] Chen, X. \& Elliott, C. M. (1994) Asymptotics for a parabolic double obstacle problem. Proc. R. Soc. Lond. A 444, 429-445.

[19] Chen, X., Hilhorst, D. \& Logak, E. (2010) Mass conserving Allen-Cahn equation and volume preserving mean curvature flow. Interfaces Free Boundaries 12, 527-549.

[20] Cucuringu, M., Pizzoferrato, A. \& VAn Gennip, Y. (2021) An MBO scheme for clustering and semi-supervised clustering of signed networks. Communications in Mathematical Sciences 19(1), 73-109.

[21] Desai, M. \& RAO, V. (1994) A characterization of the smallest eigenvalue of a graph. J. Graph Theory 18(2), 181-194.

[22] Evans, L. C. (1993) Convergence of an algorithm for mean curvature motion. Indiana Univ. Math. J. 42, 533-557.

[23] Haemers, W. H. \& Spence, E. (2004) Enumeration of cospectral graphs. Eur. J. Comb. 25(2), 199-211.

[24] Haeseler, S., Keller, M., Lenz, D. \& Wojciechowski, R. K. (2012) Laplacians on infinite graphs: Dirichlet and Neumann boundary conditions. J. Spectr. Theory 2(4), 397-432.

[25] Hein, M., Audibert, J.-Y. \& von Luxburg, U. (2005) From graphs to manifolds-weak and strong pointwise consistency of graph. In: P. Auer and R. Meir (editors), Proceedings of the 18th Annual Conference on Learning Theory (COLT), Springer, New York, pp. 470-485.

[26] Jacobs, M., MerkurJev, E. \& Esedō̄Lu, S. (2018) Auction dynamics: a volume constrained MBO scheme. J. Comp. Phys. 354, 288-310.

[27] Juditsky, A. Convex Optimization Lecture 1, available online at https://www-ljk.imag.fr/membres/ Anatoli.Iouditski/cours/convex/chapitre_1.pdf accessed 26/05/2020.

[28] LaUX, T. \& SwartZ, D. (2016) Convergence of thresholding schemes incorporating bulk effects. arXiv preprint arXiv:1601.02467.

[29] Luo, X. \& Bertozzi, A. L. (2017) Convergence analysis of the graph Allen-Cahn scheme. J. Stat. Phys. 167(3), 934-958.

[30] Merkurjev, E., Garcia, C., Bertozzi, A. L., Flenner, A. \& Percus, A. (2014) Diffuse interface methods for multiclass segmentation of high-dimensional data. Appl. Math. Lett. 33, $29-34$.

[31] Merkurjev, E., Kostić, T. \& Bertozzi, A. L. (2013) An MBO scheme on graphs for segmentation and image processing. SIAM J. Imaging Sci. 6(4), 1903-1930.

[32] Mohar, B. (1991) The Laplacian spectrum of graphs. In: Y. Alavi, G. Chartrand, O. R. Oellermann \& A. J. Schwenk (editors), Graph Theory, Combinatorics, and Applications, Vol. 2, Wiley, pp. 871-898.

[33] Mugnai, L., Seis, C. \& Spadaro, E. (2015) Global solutions to the volume-preserving meancurvature flow. arXiv preprint arXiv:1502.07232.

[34] RUbinstein, J. \& STERnBERG, P. (1992) Nonlocal reaction-diffusion equations and nucleation. IMA J. Appl. Math. 48, 249-264.

[35] Rudin, W. (1991) Functional Analysis, McGraw-Hill, New York.

[36] RuUth, S. J. \& Wetton, B. (2003) A simple scheme for volume-preserving motion by mean curvature. J. Sci. Comput. 19(1), 373-384.

[37] VAN GenNiP, Y. (2020) An MBO scheme for minimizing the graph Ohta-Kawasaki functional. $J$. Nonlinear Sci. 30, 2325-2373.

[38] Van Gennip, Y., Guillen, N., Osting, B. \& Bertozzi, A. L. (2014) Mean curvature, threshold dynamics, and phase field theory on finite graphs. Milan J. Math. 82(1), 3-65.

[39] Zhang, Y., ZhaO, Z. \& FenG, Z. (2020) A unified approach to scalable spectral sparsification of directed graphs. arXiv preprint arXiv:1812.04165.

[40] Zhou, D., Schölкopf, B. \& HofmanN, T. (2005) Semi-supervised learning on directed graphs. In: NIPS'05 Advances in Neural Information Processing Systems, pp. 1633-1640. 